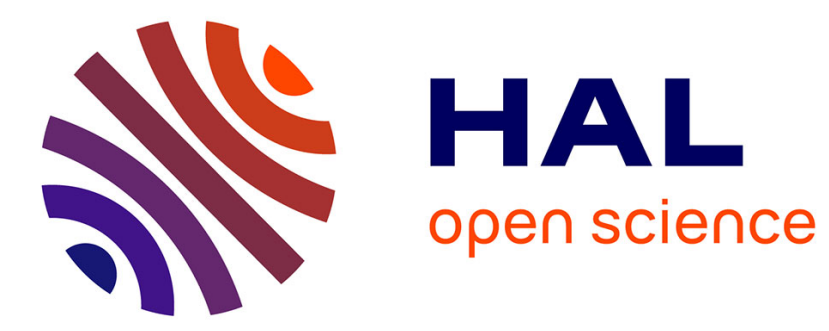

\title{
Pouvoir de marché, stratégies et régulation: Les contributions de Jean Tirole, Prix Nobel d'Economie 2014
}

\author{
David Encaoua
}

\section{- To cite this version:}

David Encaoua. Pouvoir de marché, stratégies et régulation: Les contributions de Jean Tirole, Prix Nobel d'Economie 2014. Revue d'Economie Politique, 2015, vol. 125 (1), pp.1-76. 10.3917/redp.251.0001 . halshs-01148356

\author{
HAL Id: halshs-01148356 \\ https://shs.hal.science/halshs-01148356
}

Submitted on 15 Mar 2016

HAL is a multi-disciplinary open access archive for the deposit and dissemination of scientific research documents, whether they are published or not. The documents may come from teaching and research institutions in France or abroad, or from public or private research centers.
L'archive ouverte pluridisciplinaire HAL, est destinée au dépôt et à la diffusion de documents scientifiques de niveau recherche, publiés ou non, émanant des établissements d'enseignement et de recherche français ou étrangers, des laboratoires publics ou privés. 


\title{
Pouvoir de marché, stratégies et régulation : Les contributions de Jean Tirole, Prix Nobel d'Economie 2014
}

Résumé : Cet article est consacré à une mise en perspective des travaux réalisés par Jean Tirole sur les thèmes mentionnés par le communiqué du Nobel, à savoir l'analyse du pouvoir de marché, ses modalités de contrôle et la régulation des activités de réseaux et de monopole naturel. II se développe en trois parties. La première explicite pourquoi et comment ces travaux ont participé à un profond renouveau de l'Economie Industrielle. La seconde présente les contributions participant à l'élaboration d'une véritable économie de l'innovation. La troisième partie se réfère aux travaux sur la régulation. Dans chacune de ces parties, l'accent est mis sur trois aspects: i/ l'état des connaissances au moment de ces travaux, ii/ les instruments d'analyse utilisés, et iii/ les enseignements que l'on peut en tirer. L'article cherche également à illustrer une caractéristique commune à plusieurs de ces travaux, celle de combiner, d'une part, une recherche théorique conduisant à un véritable renouvellement de nos connaissances dans différents champs de l'analyse économique, et d'autre part, un choix de questions motivées par une forte préoccupation à trouver des moyens de résolution de nombreux problèmes économiques contemporains, se fondant sur une sérieuse analyse théorique préalable.

Mots clés: oligopole, restrictions verticales, forclusion, course au brevet, systèmes non propriétaires, marchés bifaces, pools de brevets, standards technologiques, asymétries informationnelles, incitations, réseaux, régulation.

JEL : L13, L42, L51, O34

Market Power, Strategies and Regulation:

The contributions of Jean Tirole, Nobel Prize in Economics 2014

\begin{abstract}
This article is devoted to a perspective of the works of Jean Tirole on the topics mentioned by the Nobel Committee, namely the analysis of market power, its control mechanisms and the regulation of network activities and natural monopoly. It develops in three parts. The first explains why and how these works have contributed to a profound renewal of the Industrial Organization field by offering strong theoretical foundations. The second presents different contributions that advance our understanding of the Economics of Innovation. The third part refers to the works on the Economics of Regulation. In each part, the focus is on three aspects: $\mathrm{i} /$ the state of knowledge at the time of the work, ii / the analytical instruments and iii / the lessons that can be drawn. The article seeks to illustrate a common feature in many of these works, that of combining theoretical research leading to the knowledge's enlargement in various fields of economic analysis, and a choice of questions motivated by a strong concern to find solutions to some contemporary economic problems, based on a serious prior theoretical analysis.
\end{abstract}

Key words: oligopoly, vertical restraints, forclusion, patent race, non-proprietary systems, two-sided markets, patent pools, technological standards, asymmetric information, incentives, networks, regulation.

JEL : L13, L42, L51, O34 


\title{
Pouvoir de marché, stratégies et régulation : Les contributions de Jean Tirole, Prix Nobel d’Economie 2014
}

\author{
David Encaoua \\ Professeur émérite à l'Université de Paris I Panthéon Sorbonne \\ Ecole d'Economie de Paris, Centre d'Economie de la Sorbonne
}

Février 2014

\section{Introduction}

En mémoire d'Alfred Nobel, l'Académie Royale des Sciences de Suède a attribué, le 13 octobre 2014, le "Sveriges Riksbank Prize " en Sciences Economiques à Jean Tirole pour son analyse du pouvoir de marché et de la réglementation. Il est ainsi le récipiendaire du Prix Nobel d'Economie 2014. Dans son communiqué, l'Académie précisait que "JeanTirole is one of the most influential economists of our time. He has made important theoretical research contributions in a number of areas, but most of all he has clarified how to understand and regulate industries with a few powerful firms".

Cette dernière précision est très importante à rappeler dés le départ, car non seulement Jean Tirole n'est pas l'exégète d'une économie de marché libre de toute intervention publique ou l'apôtre de la "puissance du marché" comme certains commentaires l'ont laissé entendre en France ${ }^{1}$, mais ce qui lui a valu le prix Nobel, ce sont précisément des travaux qui justifient, au nom de l'intérêt général, la nécessité pour les pouvoirs publics de surveiller, contrôler et réglementer les marchés, que ces marchés soient dominés par un petit nombre d'entreprises en situation d'oligopole ou qu'ils soient en situation de monopole naturel. Les travaux de Jean Tirole sont d'autant plus importants qu'ils se situaient en rupture à la doxa dominante au moment de leur émergence. Nous y revenons plus loin, mais d'ores et déjà il est important de souligner que ces travaux, comme ceux d'autres économistes du MIT à la même époque, se situent en discordance avec la doctrine des économistes et juristes de l'Ecole de Chicago, qui considéraient que les interventions publiques en matière de politique de la concurrence comme en matière de réglementation économique, étaient souvent injustifiées et inefficaces. II revient à Jean Tirole et ses coauteurs l'immense mérite d'avoir démontré que ces croyances reposent sur des prémisses erronées ou incomplètes et qu'en cherchant à mieux comprendre les complexités de l'économie réelle, on parvenait non seulement à justifier l'intervention publique mais également et surtout à expliciter sous quelles formes il est souhaitable de la mener.

Une idée simple souvent utilisée par Tirole illustre l'originalité et la portée de cet argument. L'idée consiste à réfléchir sur le degré de validité du célèbre théorème de Coase (1960). Ce théorème stipule qu'une inefficacité dans les échanges entre deux parties, due à des externalités négatives infligées par les actions d'une des parties sur l'autre, peut être surmontée et corrigée par une négociation entre elles, pour peu que le coût de transaction de la négociation soit faible. Ainsi, selon ce théorème, l'argument selon lequel des imperfections de marché peuvent et doivent être corrigées par des interventions publiques perdrait quelque peu sa pertinence. Une des raisons invoquées par Tirole pour montrer les limites de validité de ce raisonnement réside dans le fait que les deux parties ont en général des informations privées conduisant à une impossibilité de parvenir à une issue socialement acceptable à l'issue de négociations décentralisées. Pour illustrer, prenons l'exemple d'une inefficacité de base, celle qui relie un monopole aux consommateurs. En vendant son bien au prix auquel la recette marginale égalise le coût marginal, le monopole maximise son profit et permet à certains consommateurs (ceux dont la disponibilité à payer est supérieure au prix de monopole) de s'approprier un certain surplus. II n'en reste pas moins que même si le résultat est un optimum de Pareto pour les deux parties (le monopole et ses clients) une inefficacité flagrante subsiste, en ce sens que cet optimum entre agents n'est pas un optimum social : une part du surplus social global est "perdue", au sens où elle n'est appropriée ni par le monopole, ni par les consommateurs (cette part est désignée en anglais comme le "deadweight loss"). Cette inefficacité disparaitrait si le monopole connaissait la disponibilité à payer de chaque consommateur, de sorte qu'il vendrait son bien à des prix personnalisés, pratiquant ainsi une discrimination du premier degré, conduisant à ce qu'aucune perte du surplus social ne subsiste. Ce qui empêche de le faire, c'est bien que le monopole ne dispose pas de ces informations individualisées et qu'il ne peut les obtenir par une quelconque négociation, bien qu'il connaisse la fonction de demande qui s'adresse à lui. Voilà une asymétrie informationnelle de base, dont ne tient pas compte le théorème de Coase et qui ne peut être corrigée par une négociation directe entre le monopole et les consommateurs de son produit. Comme nous le verrons par la suite, c'est loin d'être l'unique circonstance où le théorème de Coase ne s'applique pas. $^{2}$

Les motivations générales des travaux qui ont valu l'attribution du prix Nobel à Jean Tirole peuvent ainsi être résumées en une double exigence : d'une part, réexaminer les stratégies de concurrence imparfaite des entreprises, à la base de leur pouvoir de marché, et analyser dans quelle mesure elles conduisent à distordre le fonctionnement des marchés dans nos économies; d'autre part, mener une réflexion approfondie afin de proposer des modalités d'interventions publiques appropriées pour corriger ces distorsions.

Au-delà du rappel de ces motivations générales, il s'agit d'être un peu plus précis, et pour cela, nous consacrons cet article à une mise en perspective des travaux réalisés par Jean Tirole sur les thèmes mentionnés par le communiqué du Nobel, en mettant l'accent sur trois aspects : i/ l'état des connaissances au moment de ces travaux, ii/ les instruments d'analyse utilisés, et iii/ les enseignements que l'on peut en tirer.

Même circonscrite de la sorte, la mission reste difficile, périlleuse, et en tout état de cause, délicate pour plusieurs raisons.

D'abord, du fait du nombre impressionnant de contributions, réalisées seul ou avec une liste de co-auteurs célèbres. Le CV de Jean Tirole mentionne 180 publications en anglais dans les revues internationales les plus prestigieuses, 38 publications en français, 12 ouvrages traduits en plusieurs langues, sans compter les rapports sur différentes questions d'intérêt public et les multiples "Key Note Lectures"

\footnotetext{
${ }^{1}$ Certains commentateurs, pas nécessairement malveillants mais ayant abusivement traduit l'expression "market power" par puissance du marché (au lieu de pouvoir de marché) en ont déduit que le prix Nobel 2014 était un apôtre d'un marché libre et puissant !

${ }_{2}^{2}$ Par exemple, lorsque c'est la structure des prix qui importe et non plus simplement leur niveau, comme c'est le cas dans les marchés biface examinés plus loin, l'intermédiation des relations entre deux groupes d'agents au travers d'une plateforme commune peut être la forme sociale privilégiée.
} 
données de par le monde. Autrement dit, le Nobel d’Economie 2014 n'a pas été attribué pour une découverte spécifique, mais pour une œuvre gigantesque dont chaque travail porte en lui une innovation importante. Lors de la cérémonie de remise de la médaille d'or du CNRS à Jean Tirole en 2007, son directeur de thèse au MIT, qui se trouve être son ami et l'un de ses nombreux co-auteurs, Eric Maskin, ironisait déjà sur une possible supercherie tant la profusion de l'œuvre et sa qualité lui paraissaient considérables ! II ajoutait, non sans malice, que Jean lui avait rendu un mauvais service, car n'ayant quasiment jamais intervenu dans l'avancement des travaux de la première thèse qu'il était censé diriger, il en avait naïvement conclu qu'il en serait de même pour les suivantes, ce qui évidemment ne pouvait pas manquer d'être source de déconvenues par la suite !

La deuxième raison pour laquelle la mise en perspective des contributions de Jean Tirole est très difficile à réaliser est que ses travaux couvrent en fait un très grand nombre de questions à l'intérieur de différents grands thèmes, au point qu'il est impossible d'être exhaustif. On distingue au moins sept grands champs de l'économie auxquels Jean Tirole a apporté des contributions décisives: i/ l'analyse des comportements stratégiques dans des situations de concurrence imparfaite et les conséquences en matière de politique de la concurrence; ii/ les analyses en amont du fonctionnement des marchés, qui concernent la recherche, le développement, la technologie, la propriété intellectuelle, et tout ce que l'on regroupe sous la bannière de l'économie de l'innovation; iii/ les théories de la régulation des activités de réseaux et des monopoles naturels qui fondent les modalités d'intervention de l'Etat dans les services publics; iv/ la théorie des contrats et des organisations qui fournit un cadre intellectuel original pour éclairer de multiples questions, notamment celles montrant pourquoi certaines défaillances de marchés peuvent être résolues par recours à des formes de coopération contractuelle; v/ la théorie des comportements exubérants sur les marchés boursiers et financiers, qui se traduisent notamment par la formation de bulles spéculatives sur ces marchés; vi/ la régulation bancaire et financière pour éviter les risques de crise systémique; vii/ l'analyse des comportements au croisement de la psychologie et de l'économie. Bien entendu, nous ne couvrirons pas l'ensemble de ces sept champs ici. Le document produit par le Comité d'attribution du Prix Nobel en Sciences Economiques de l'Académie Royale des Sciences de Suède pour présenter les principales contributions de Jean Tirole ${ }^{3}$ ne comporte pas moins de 54 pages, tout en reconnaissant que seules les contributions en lien direct avec les motifs retenus par le Nobel sont recensées. Notre ambition sera encore plus modeste : le choix des travaux présentés est assez restreint et reflète à la fois des préférences spécifiques, des limites individuelles en termes de capacité d'absorption et des contraintes éditoriales évidentes.

Nous n'examinerons dans cet article que trois des sept thèmes que couvrent les travaux de Jean Tirole, ceux qui lui ont précisément valu le prix Nobel. Le premier thème porte sur l'analyse des comportements stratégiques et de la dynamique de la concurrence sur des marchés oligopolistiques, analyse qui a profondément contribué à renouveler les fondements théoriques de l'Economie Industrielle et à dégager des ramifications significatives en termes de politiques de la concurrence. Le deuxième thème porte sur une dimension spécifique de la concurrence, celle qui a lieu en amont du marché et qui concerne la R\&D, l'innovation, la propriété intellectuelle et toutes les modalités de la concurrence pour le marché et non plus seulement sur le marché. Le troisième thème enfin concerne la régulation des monopoles naturels et des activités économiques de réseaux pour dégager les modalités souhaitables de cette régulation. Les apports de Jean Jacques Laffont et de Jean Tirole sur ce troisième thème ont été décisifs, tant par le renouvellement théorique que ces travaux ont permis que par leur impact sur les mécanismes de régulation sectorielle, notamment dans les activités de réseaux.

Nous traiterons ces trois thèmes en essayant de répondre à trois types de questions : 1 . Comment les développements de la théorie des jeux, auxquels Jean Tirole a activement participé, ont permis un renouvellement de l'analyse du pouvoir de marché, au travers de divers mécanismes de concurrence oligopolistique, conduisant à une restructuration complète de nos connaissances dans un pan entier de l'Economie, à savoir celui de l'Economie Industrielle ? 2. Comment la théorie des incitations et du "mechanism design" est parvenue à revitaliser les approches économiques de la réglementation économique des monopoles naturels et de la commande publique ? 3. Comment les contributions théoriques ont-elles influencé les politiques effectives des pouvoirs publics en matière de politique de la concurrence, de réglementation sectorielle et de protection intellectuelle?

L'article se déroule ainsi selon un plan en trois parties. La première partie est consacrée aux travaux de Jean Tirole qui ont porté sur les comportements de concurrence imparfaite et notamment de la concurrence oligopolistique. Ils portent sur des thèmes majeurs de l'Economie Industrielle et ils ont profondément contribué à renouveler cette discipline. Ce sont aussi bien des travaux individuels que menés en collaboration avec divers auteurs comme Drew Fudenberg, Eric Maskin et Patrick Rey. Le cœur de ces travaux concerne l'analyse du pouvoir de marché, c'est-à-dire la capacité de certains agents du secteur productif d'infléchir les prix, les structures et les comportements en faveur de leurs intérêts et non de l'intérêt général comme l'aurait prescrit la main invisible. L'ouvrage fondamental de Jean Tirole "Industrial Organization" publié en 1988 permettait déjà de percevoir l'étendue et la pertinence de ce renouvellement. Plus de 25 ans après sa publication, il reste l'ouvrage de référence en matière d'enseignement de cette discipline et, comme le remarque le document du Comité Nobel auquel on a fait référence plus haut, ce qui ne figure pas dans l'ouvrage de 1988, est souvent dû à des contributions ultérieures de l'auteur lui-même.

La deuxième partie regroupe des contributions portant sur les comportements stratégiques, non plus en termes de variables directement liées au fonctionnement des marchés comme le sont les prix et les quantités, mais plutôt en amont du marché, comme c'est le cas pour la Recherche et Développement, l'innovation, les nouvelles technologies et la propriété intellectuelle. Nous avons regroupé ces contributions pour illustrer la présence significative de Jean Tirole sur des problématiques contemporaines. Elles portent sur des composantes essentielles de ce qu'on pourrait appeler "l'économie de l'innovation". On se réfère ici à des questions liées à la protection intellectuelle, aux courses au brevet, à la mise en commun de plusieurs brevets, à la diffusion et l'adoption des nouvelles technologies, aux marchés biface, aux activités en réseaux, aux standards technologiques et aux méthodes de commercialisation des licences, aux logiciels libres, etc. Là encore, seul ou en collaboration avec d'autres auteurs dont Drew Fudenberg, Richard Gilbert, Josh Lerner, Patrick Rey, Jean Charles Rochet et Joseph Stiglitz, les travaux de Jean Tirole ont ouvert des pans entiers de la recherche économique contemporaine la plus active. La troisième partie enfin concerne la théorie de la régulation économique qui comprend la régulation des monopoles naturels, celle des activités de réseaux et les modalités de la commande publique. Sur ce thème, les contributions sont pour la plupart effectuées avec Jean Jacques Laffont et leur monumental ouvrage de 1993 présente la synthèse de près de dix années de travaux en commun. ${ }^{4}$ En particulier, l'article de 1986 publié dans le Journal of Political Economy représente une avancée considérable dans la mesure où, mettant l'accent sur les asymétries d'information entre le régulateur et l'entreprise régulée, il parvient à présenter un mécanisme révélateur et socialement optimal particulièrement simple, dont la principale caractéristique est de conduire à la nécessité d'un compromis entre un objectif d'efficacité productive (inciter l'entreprise régulée à réduire son coût de production) et un objectif d'efficacité redistributive (éviter que

\footnotetext{
${ }^{3}$ Economic Sciences Prize Committee of the Royal Swedish Academy of Sciences, Scientific Background on the Sveriges Riksbank Prize in Economic Sciences in Memory of Alfred Nobel 2014, JEAN TIROLE: MARKET POWER AND REGULATION, 13 October 2014

${ }^{4}$ On peut raisonnablement penser que si Jean Jacques Laffont était encore des nôtres, il aurait vraisemblablement partagé avec Jean Tirole la plus haute distinction.
} 
l'entreprise régulée ne s'accapare une rente trop importante au détriment des consommateurs). Ce mécanisme, qui prend en compte le coût des fonds publics censés représenter le coût social d'un éventuel financement de l'entreprise régulée, est devenu avec le travail antérieur de David Baron et Roger Myerson (1982), la référence incontournable en matière de régulation économique. Les contributions postérieures ont élargi le cadre d'analyse en intégrant divers phénomènes importants tels que la capture du régulateur, les spécificités institutionnelles ou encore les aspects dynamiques des contrats. De plus, le souci de mieux comprendre les activités de réseaux, là où les questions de l'accès aux infrastructures et de tarification des services sont proéminentes, a conduit à des analyses dont l'impact sur la régulation économique est maintenant bien établi. Sur ce troisième thème, les contributions de Jean Tirole, en collaboration avec Jean Jacques Laffont, et d'autres auteurs dont Bernard Caillaud, Xavier Freixas, Roger Guesnerie, Oliver Hart, Bengt Holmström, Patrick Rey, ont également été très importantes.

Les apports de ces divers travaux aux politiques d'intervention publique sont examinés au travers de trois supports : les politiques de la concurrence, de la propriété intellectuelle et de la régulation sectorielle. Une citation extraite du discours de réception, prononcé par Jean Tirole, résume bien l'esprit qui a présidé à l'élaboration de ces apports aux politiques publiques: "Recognizing that industries are different from each other and evolve rapidly, researchers in industrial organization have patiently built a body of knowledge that has helped regulators to better understand market power and the effects of policy interventions, and helped firms to formulate their strategies. They have thereby contributed to making this world a better place, the economist's first mission. Yet, there is so much we still have to learn, and the world changes faster than our understanding can keep up."

Concernant les politiques de la concurrence, au moins deux impacts importants peuvent être retenus. Premièrement, en montrant que la correction publique des imperfections de marché ne saurait être opérée par l'édiction d'interdits universels, valables en toutes circonstances, les travaux de Jean Tirole ont contribué à faire progresser les approches dites au cas par cas, que l'on regroupe sous le vocable de "règle de raison", de préférence aux approches globalisantes s'appuyant sur des "règles per se ". Contrairement à la règle universelle dite du "one size fits all" qui prévaut tant bien que mal pour le droit des brevets par exemple, Jean Tirole a beaucoup contribué à ce que ce ne soit plus le cas en droit de l'antitrust, faisant plutôt prévaloir l'adage du "one size doesn't fit all". Les particularités de chaque industrie et la singularité des situations traitées sont mises en valeur pour aboutir à une meilleure distinction entre ce qui devrait être permis et ce qui est plutôt délictuel. Deuxièmement, en matière de traitement des "restrictions verticales" , c'est-à-dire celles qui figurent dans les relations entre l'amont productif et l'aval distributif, les travaux de Jean Tirole, en collaboration avec Patrick Rey, ont profondément transformé aussi bien les références à l'Ecole Structuraliste de Harvard que celles émises en réaction par l'Ecole de Chicago, qui imprégnaient jusque là les principes de la politique de la concurrence en matière de pratiques verticales restrictives.

Concernant la propriété intellectuelle, les travaux de Jean Tirole, menés conjointement avec Josh Lerner, ont amené à reconsidérer des questions importantes sur les complémentarités ou les conflits entre la politique de la concurrence et la politique de l'innovation. Trois questions particulières ont retenu notre attention. La première a trait au traitement juridique de la mise en commun d'un paquet de brevets ("patent pool"), s'apparentant, pour certains à un comportement collusif et, pour d'autres, à une exigence d'efficacité en termes d'innovation. La deuxième question porte sur le traitement des brevets dits "essentiels" dans les standards technologiques. De multiples litiges juridiques prévalent à propos de l'usage de ces brevets, notamment au travers des règles de licences. Là encore des solutions inspirées par les travaux de Josh Lerner et Jean Tirole commencent à être mises en place. La troisième question porte sur les motivations des acteurs en termes de logiciels non propriétaires ("open systems") et des restrictions d'usage en matière de licences.

Enfin, concernant la régulation des activités de réseaux, quatre principes, issus des travaux théoriques menés en collaboration avec Jean Jacques Laffont et Patrick Rey, sont maintenant largement appliqués: 1. Nécessité de mettre en place des agences de régulation indépendantes; 2 . Importance des mécanismes de régulation reconnaissant le rôle des incitations pour réduire à la fois les coûts de production et la rente de l'entreprise régulée ; 3 . Rôle important de l'ouverture à la concurrence par l'attribution de licences pour l'exploitation de ressources rares ; 4 . Enfin, introduction de règles favorisant à la fois le rééquilibrage des prix et les obligations de service universel.

Le plan détaillé se présente comme suit. La section II cherche à illustrer comment et pourquoi les contributions de Jean Tirole ont permis un renouvellement complet de l'Economie Industrielle. Après un rappel de l'héritage (II.1) et des raisons qui ont fait le succès du manuel de base de 1988 (II.2), la sous-section suivante présente des contributions en matière d'analyse des investissements stratégiques lorsque ceux-ci sont suivis d'une concurrence de court terme (II.3) avant de présenter des modèles dynamiques d'oligopole où la concurrence est de long terme (II.4). Forts de ces premières analyses de la concurrence oligopolistique, une évaluation des contributions de Jean Tirole à l'Economie industrielle en tant que discipline est présentée en tenant compte des critiques adressées à cette approche (II.5). La sous section suivante examine les contributions concernant les relations verticales (II.6) avant d'en évaluer les impacts en matière des politiques de la concurrence (II.7).

La section III reprend des travaux traitant de comportements qui se situent en amont du marché des biens et qui concernent la R\&D, la technologie, les réseaux et la propriété intellectuelle. On examine successivement les contributions en matière de courses au brevet (III.1), de logiciels libres (III.2), de marchés bi-face (III.3) et de pools de brevets et de standards technologiques (III.4).

La section (IV) reprend quelques uns des travaux de Laffont et Tirole ayant contribué à refonder complètement l'approche de la régulation économique. Nous commençons par rappeler les travaux ayant conduit à la nécessité de réguler les activités de réseaux (sous section IV.1), I'héritage en matière de théories de la régulation des monopoles naturels (IV.2) et les principales modalités de contrôle d'un monopole naturel (IV.3). Nous procédons ensuite à une brève présentation du modèle de base de Jean Jacques Laffont et Jean Tirole (sous section IV.4) avant d'en examiner quelques prolongements (sous section IV.5), et de présenter des questions institutionnelles (sous section IV.6). La conclusion fait l'objet de la section VI.

\section{Pouvoir de marché et stratégies d'entreprises : l'Economie Industrielle revisitée par Tirole}

\section{II.1 L'héritage en matière de stratégies d'entreprises en oligopole}

Pour apprécier la valeur ajoutée que les contributions de Jean Tirole ont apporté à l'Economie Industrielle, il est utile de consacrer quelques lignes à l'état de cette discipline jusqu'aux années 1970 . Un ouvrage de référence parmi les économistes industriels de cette génération était celui de Michael Scherer dont la première édition date de 1970 et la seconde de 1980. II définissait ainsi la discipline dans l'introduction de son ouvrage : "the field is concerned with how productive activities are brought into harmony with society's demands for goods and services through some organizing mechanism such as a free market, and how variations and imperfections in the organizing mechanism affect the degree of success achieved by producers in satisfying society's wants". Le maitre mot dans cette citation est celui des 
imperfections dans le fonctionnement des marchés. Sur le plan de l'analyse théorique de ces imperfections, figure évidemment l'héritage glorieux des économistes de la concurrence imparfaite, du XIXème et début du XXème siècle, parmi lesquels on peut citer des ingénieurs économistes français tels que Antoine Augustin Cournot et Jules Dupuit, des mathématiciens comme Joseph Bertrand et Frank Ramsey, et des économistes anglais et allemands tels que Edward Chamberlin, Francis Edgeworth, Harold Hotelling, Joan Robinson et Heinrich von Stackelberg. Mais c'est surtout sur le plan des travaux empiriques que l'Economie Industrielle de la première génération a prospéré, au travers du triptyque "Structure - Comportements - Performance" (SCP) que l'Ecole de Harvard, sous la férule de Edward Mason (1939), puis de Joe Bain (1956) et de bien d'autres, avait développé. Je ne reviens pas ici sur le contenu de ce triptyque bien connu, sinon pour dire que la plupart des contributions empiriques de cette période ont cherché à expliquer le taux de profit des branches (ou parfois le taux de marge, représentation approximative de l'indice de Lerner en tant que mesure du pouvoir de marché) par des variables de structure de ces branches, parmi lesquelles deux d'entre elles étaient des pièces maitresses, la concentration industrielle et les barrières à l'entrée. La corrélation positive entre le taux de marge et la concentration des branches (part de marché cumulée des quatre premières entreprises ou indice d'Herfindahl) était considérée comme une preuve que le pouvoir de marché que détenaient les entreprises de la branche était d'autant plus élevé que la concentration de la branche était forte. ${ }^{5}$ Autrement dit, d'une corrélation positive on passait facilement à une relation de causalité ! Une des grandes faiblesses de cette approche est que le triptyque initial SCP s'est vite transformé en un diptyque SP, faisant pratiquement l'impasse sur les variables de comportement. Celles-ci faisaient parfois l'objet d'études de cas cherchant à illustrer l'effet sur l'évolution des structures de marché, des choix stratégiques des entreprises, notamment en termes d'accroissement de leurs capacités de production pour s'adapter aux rendements croissants. Sur le plan des politiques d'intervention publique, les grandes lois à l'origine du droit de la concurrence, telles le Sherman Act (1890) et le Clayton Act (1914) aux Etats Unis, ont cherché à s'attaquer aux trusts, accusés d'être à l'origine de deux phénomènes collectivement dommageables: d'une part, des prix excessivement élevés résultant de pratiques collusives durant les périodes de croissance et, d'autre part, une grande vulnérabilité aux retournements de conjoncture du fait de capacités de production largement sous utilisées en situation de récession. Après une courte période de mise en sommeil de la politique de la concurrence de 1915 à 1936, une seconde période a vu le jour caractérisée par une politique de la concurrence très active, jusqu'au milieu des années 1970, notamment sous l'influence de l'Ecole structuraliste de Harvard. Les présupposés à l'encontre de la concentration industrielle ont joué un rôle déterminant durant cette période (David Encaoua et Roger Guesnerie, 2006).

Ces excès n'ont pas manqué de soulever à partir des années 1970 une très forte réaction, de la part de juristes (Aaron Director), d'économistes (Harold Demsetz, George Stigler) et de juges (Richard Posner, Robert Bork et Frank Easterbrook), tous rattachés au lieu d'où ces critiques ont été initiées, l'Ecole de Chicago. En même temps que ces critiques remettaient en cause l'utilisation abusive de la variable concentration, en lui attribuant un rôle causal, alors qu'elle aurait dû elle-même être endogénéisée, ils contestaient les effets présupposés négatifs des pratiques que l'Ecole de Harvard qualifiait de "pratiques restrictives à la concurrence". Enfin ils mettaient en avant le rôle fondamental de la concurrence potentielle exercée par d'éventuels entrants pour discipliner les concurrents en place. Leur jugement à l'égard de l'Ecole structuraliste de Harvard est bien traduite par le titre de l'ouvrage critique que Robert Bork (1978) leur consacrait : "The Antitrust Paradox: A Policy at War With Itself". Ainsi, selon la critique de l'Ecole de Chicago, loin de chercher l'efficacité économique, la politique de la concurrence telle qu'elle était menée sous les auspices de l’Ecole structuraliste de Harvard allait à l'encontre de cette efficacité. Sur le plan des idées, l'influence de l'Ecole de Chicago sur l'antitrust est longtemps restée déterminante.

Mais à nouveau, le caractère excessif de la critique adressée par l’Ecole de Chicago a eu pour effet de faire émerger une reconstruction des fondements de la discipline, dans laquelle le débat ne se situerait plus entre, d'un côté, les tenants d'une approche structuraliste où les structures des marchés seraient à l'origine des distorsions économiques et, de l'autre côté, les tenants d'une approche où ce serait l'efficacité économique plutôt que la recherche du pouvoir de marché qui guiderait les choix des entreprises. L'exigence d'une reconstruction, et non pas d'une synthèse, est au cœur des travaux de Jean Tirole et de ses coauteurs, et s'il fallait leur attribuer un label commun, cela pourrait être celui d'"Ecole de la Théorie des Jeux", dont l'influence a commencé à rayonner à partir du MIT au cours des années 1980 .

Quelques mots initiaux sur la formation du Nobel d’Economie 2014 peuvent être éclairants pour le lecteur. Jean Tirole est Polytechnicien (X 1976), Ingénieur du Corps des Ponts et Chaussées (1978). Après avoir obtenu un doctorat de $3^{\text {ème }}$ cycle en Mathématiques de la Décision à Paris IX Dauphine en 1978 ("Essais sur le calcul économique public et sur le taux d'actualisation"), il part aux Etats-Unis préparer un PhD au MIT, titre qu'il obtient en 1981 ("Essays in Economic Theory"). Il revient en France où il poursuit ses recherches de 1981 à 1984 au CERAS, à l'Ecole Nationale des Ponts et Chaussées, avant de repartir au MIT où il devient Professeur (1984-1991). De 1981 au début des années 1990, il a publié une série d'articles consacrés à différents thèmes de l'Economie Industrielle, dont l'un, méthodologique, est paru dans la Revue d'Economie Politique : "Jeux Dynamiques: un Guide de l'Utilisateur," (REP, 1983, 93: 551-575). Durant cette période, il a également enseigné en France, notamment un cours d'Economie Industrielle à l'ENSAE, dont les Notes de Lecture ont été publiées en France sous le titre "Concurrence Imparfaite" (1985). Ces notes ont ensuite été enrichies de toutes les contributions réalisées durant ces années pour devenir en 1988 un manuel de base pour l'enseignement de cette discipline. Le manuel, paru en 1988, aura un succès immédiat et retentissant. En 1991, Jean Tirole est de retour en France et s'installe définitivement à Toulouse. Une très étroite collaboration avec Jean Jacques Laffont s'instaure, jusqu'au décès de ce dernier. II participe activement à la construction de l'Ecole d'Economie de Toulouse, connue et respectée de par le monde et met en place I'IAST (Institute of Advanced Studies of Toulouse) qui développe des programmes de recherche interdisciplinaire.

\section{II.2 Un manuel qui a renouvelé le champ disciplinaire}

Commençons par quelques mots de synthèse pour expliquer pourquoi les contributions de Jean Tirole à l'Economie Industrielle ont été essentielles à la refondation de la discipline. Le manuel de Jean Tirole rassemble, sous une forme pédagogique tout à fait remarquable, la plupart des travaux qui ont contribué, depuis le milieu des années 1970 à la fin des années 1980, à attribuer un nouveau statut à la discipline. Celle-ci s'est trouvée en effet investie d'un statut scientifique beaucoup plus appréciable qu'elle ne l'était auparavant, attirant de ce fait de nombreux étudiants brillants, qui à leur tour ont continué de faire progresser ce champ d'analyse. Pour de nombreux économistes, tout s'est passé comme si le manuel de Jean Tirole était à l'origine d'un cercle vertueux : la mise en évidence de l'intérêt du sujet a permis l'émergence d'un champ disciplinaire spécifique que l'on rattache plutôt à la microéconomie, mais qui à la différence de celle-ci ne traite pas les entreprises, les marchés et les technologies comme des objets exogènes, mais en fait plutôt les principaux objets d'étude. De plus, faut-il le préciser, même si de nombreuses écoles de commerce utilisent des travaux de Jean Tirole dans leurs enseignements, l'économie industrielle se distingue fondamentalement de la théorie dite de la "Business Strategy", en ce sens qu'à la

\footnotetext{
${ }^{5}$ Ce qui laissait penser que les résultats d'une concurrence en quantités (modèle de Cournot) étaient plus compatibles avec ce qu'on observait que les résultats d'une concurrence en prix (modèle de Bertrand).
} 
différence de cette dernière, elle met au centre de ses préoccupations le bien être collectif et qu'elle cherche pour cela les moyens d'intervention publique les plus adéquats pour y parvenir.

Avant d'entrer dans le vif du contenu des contributions de Jean Tirole, essayons de comprendre pourquoi le manuel de Jean Tirole est devenu, selon pratiquement tous les économistes de la discipline, un instrument aussi indispensable pour le débutant comme pour le chercheur confirmé. Premièrement, c'est un livre où l'accent premier porte sur l'analyse économique des questions traitées et non sur l'aspect technique des méthodes utilisées. Certes, celles-ci jouent un rôle unificateur de la discipline dans son ensemble, mais elles sont toujours au service de l'essentiel, celui-ci consistant à comprendre les fondamentaux de la question traitée afin de dégager les intuitions premières. Une bonne partie du "savoir faire" de Jean Tirole est de découvrir quels sont ces fondamentaux et de les réunir autour de quelques intuitions premières avant d'en proposer une modélisation inventive et précise. L'étudiant qui aura compris ces fondamentaux sera plus à même de faire l'effort pour maîtriser ensuite les outils d'analyse. Deuxièmement, l'ouvrage présente en première partie des questions afférentes aux dimensions du pouvoir de marché d'une entreprise en monopole (en l'absence donc d'un quelconque aspect stratégique dans le fonctionnement du marché) avant d'examiner dans une deuxième partie les mêmes questions ou le plus souvent des questions plus spécifiques, dans les marchés constitués d'un "petit nombre" d'entreprises où les aspects stratégiques deviennent alors centraux. C'est là, me semble-t-il, un artefact pédagogique qui consiste justement à éviter que les aspects techniques de la théorie des jeux ne prévalent sur le raisonnement économique. Troisièmement, un autre artefact sur le plan pédagogique consiste à distinguer dans chaque chapitre ce qui relève du tronc commun et ce qui relève d'aspects plus avancés, présentés dans une section dite supplémentaire. Là encore, cette façon de faire est utile dans différents enseignements selon le degré d'avancement des étudiants. Enfin, un point important qu'il convient de souligner est que le manuel de Jean Tirole est utilisé de par le monde non seulement pour enseigner l'Economie Industrielle, mais également et surtout par les chercheurs les plus en pointe qui trouvent là les points nodaux à partir desquels leur travail se développe. Nous passons en revue dans ce qui suit quelques uns des travaux de Jean Tirole qui concernent les aspects stratégiques de la concurrence oligopolistique.

\section{II.3 Engagement stratégique et concurrence de court terme}

\section{a/ Les instruments d'analyse}

Jusqu'à la fin des années 1970, l'analyse théorique des comportements stratégiques en concurrence imparfaite, notamment le comportement adopté par une firme en place face à la menace d'entrée d'un concurrent, était caractérisée par des hypothèses plutôt adhoc, assez difficilement justifiables. L'une d'elles, la plus célèbre, consistait à supposer qu'une entreprise en situation de monopole sur son marché, avait le pouvoir de dissuader l'entrée d'un concurrent potentiel en choisissant, avant l'entrée, un prix inférieur au coût de production unitaire de l'entrant. C'est I'hypothèse, dite du "prix limite", attribuée à trois auteurs Joe Bain, Paolo Sylos Labini et Franco Modigliani. Cette hypothèse (dite BLM) a beaucoup été utilisée, tant sur le plan des analyses théoriques que sur celui des prescriptions en termes de droit de la concurrence (comportement de prédation).

La question longtemps restée lettre morte, était de savoir si, pour l'entrant potentiel, il était justifié de penser que s'il venait à entrer, la firme en place aurait intérêt à maintenir ce prix ou ce niveau de production. La réponse est évidemment non car, si l'entrée se réalise, l'entreprise en place gagne à s'adapter à la nouvelle situation post entrée. Cela implique que la stratégie mise en place avant l'entrée est une menace non crédible, car il n'est pas dans l'intérêt de l'agent qui la brandit ex ante de la mettre à exécution ex post. Cette critique de I'hypothèse BLM est d'autant plus justifiée que le niveau de production ou de prix, que la firme en place est censée utiliser comme une arme pour dissuader l'entrée d'un concurrent, porte en fait sur une variable de court terme facilement réversible. Les prémisses du prix limite souffraient donc de deux défauts majeurs. D'une part, le prix choisi avant l'entrée ne constitue pas une menace crédible pour dissuader un concurrent potentiel d'entrer sur le marché. D’autre part, le prix limite ne comporte aucune irréversibilité pouvant lui conférer un rôle d'engagement. Ces critiques ont contribué à un renouveau des analyses des comportements stratégiques en concurrence imparfaite (David Encaoua, Paul Geroski et Alexis Jacquemin, 1986).

Ce renouveau a été justifié par l'introduction en théorie des jeux de trois raffinements très opératoires de la notion centrale d'équilibre de Nash. Rappelons ici qu'un équilibre de Nash d'un jeu à $n$ joueurs désigne un $n$-uple de stratégies telles que chacune d'elles soit une meilleure réponse aux (n-1) autres ou, dit autrement, un équilibre de Nash est un n-uple de stratégies dont aucun joueur n'a intérêt à dévier unilatéralement. II est plus aisé de donner la définition d'un équilibre de Nash pour un jeu sous forme normale, comme nous le faisons ici, que pour un jeu défini sous forme extensive, alors que comme nous le verrons, c'est la forme extensive qui est la plus adaptée à l'analyse des comportements stratégiques.

Le premier raffinement résulte d'une critique de base à l'encontre de l'équilibre de Nash d'un jeu en information complète comportant plusieurs étapes successives. Un tel jeu conduit en général à plusieurs équilibres de Nash dont certains sont très peu vraisemblables. Prenons l'exemple d'un jeu à deux étapes où, à la première étape, une firme en place a le choix entre deux stratégies : Agressive (A) ou Coopérative (C), et à la deuxième étape, l'entrant potentiel choisit, au vu de la stratégie adoptée par la firme en place à l'étape antérieure, soit de ne pas entrer (NE), soit d'entrer (E). Dans ce dernier cas une concurrence de court terme a lieu entre la firme en place et l'entrant. Supposons qu'en cas d'entrée, le gain de la firme en place soit plus élevé si elle adopte une stratégie coopérative $C$ plutôt qu'une stratégie agressive A. Si nous explicitons la forme normale de ce jeu, il est alors facile de construire des fonctions de gain telles que le couple (A, NE) soit un équilibre de Nash du jeu sous forme normale. Mais en retournant à la forme extensive du jeu qui prend en compte le caractère séquentiel des décisions, le couple (A, NE) n'apparait pas comme un équilibre plausible car il n'est pas un équilibre de Nash du jeu post entrée de la deuxième étape. Reinhard Selten (1975) devait ainsi proposer un raffinement conceptuel important de la notion d'équilibre de Nash : il s'agit de l'équilibre parfait-en sous jeux (EPSJ) défini comme un équilibre de Nash de tout sous jeu du jeu initial. Ce concept très opérationnel a joué un rôle crucial dans la notion d'engagement stratégique en situation de concurrence de court terme (Drew Fudenberg et Jean Tirole, 1984) que nous examinons plus bas.

Le deuxième raffinement de l'équilibre de Nash résulte de l'introduction du rôle de l'information. Un jeu bayésien est un jeu sous forme normale dans lequel l'information dont dispose chaque joueur sur les caractéristiques des autres joueurs est incomplète. C'est en particulier le cas d'un jeu dans lequel un ou plusieurs joueurs sont incertains quant aux gains des autres joueurs ou quant à leurs caractéristiques idiosyncratiques. Dans la théorie de l'agence, on désigne les caractéristiques possibles d'un agent comme définissant l'ensemble de ses "types". Chaque agent connait son type mais ne connaît pas précisément les types des autres. II dispose néanmoins de croyances initiales sous la forme d'une distribution de probabilités sur les types possibles des autres joueurs. La stratégie d'un agent dépend de son type. La notion d'équilibre bayésien, introduite par John Harsanyi (1967-68), est un raffinement de l'équilibre de Nash. Un agent de type donné choisit sa meilleure réponse aux stratégies des autres joueurs en maximisant son espérance de gain, espérance calculée par rapport aux croyances qu'il a sur les types des autres joueurs. La formalisation des jeux bayésiens a constitué une étape cruciale aussi bien pour la théorie de l'agence que plus généralement pour la théorie du "mechanism design". 
Le troisième raffinement de la notion d'équilibre de Nash est en fait une combinaison des deux premiers. II s'agit de l'Equilibre Séquentiel, introduit par David Kreps et Robert Wilson (1982) et dont les liens avec l'Equilibre Bayésien Parfait (EBP) ont été analysés par Drew Fudenberg et Jean Tirole (1991). Le concept d'EBP prend en compte à la fois la dynamique séquentielle des jeux à plusieurs étapes et l'information incomplète sur les types des joueurs. Le point nouveau est qu'au sommet de tout sous jeu, les croyances d'un joueur sur les types des autres joueurs sont révisées à partir des croyances initiales de manière à être compatibles avec les stratégies qui ont mené au sommet du sous jeu. La révision des croyances se fait selon la règle de Bayes en fonction des choix opérés par les autres joueurs au cours des étapes antérieures. Le concept d'équilibre bayésien parfait est ainsi formé d'un couple (stratégies, croyances) défini en tout sous jeu, satisfaisant aux propriétés suivantes : i/ Etant données les croyances, les stratégies forment un équilibre parfait pour tous les sous jeux issus du jeu initial ; ii/ Dans tout sous jeu, les croyances révisées actualisent les croyances antérieures en fonction des stratégies ayant mené au sous jeu par application du théorème de Bayes, ce qui implique que les croyances révisées au sommet d'un sous jeu soient compatibles avec les stratégies qui ont conduit au sous jeu. Les travaux de Jean Tirole ont tiré un remarquable parti de ces avancées de la théorie des jeux, et ont même contribué à produire de nouvelles avancées conceptuelles de cette théorie.

\section{b/ Typologie des investissements stratégiques et concurrence de court terme.}

Comment une entreprise en place peut-elle influencer ses concurrents, sachant qu'un investissement stratégique a nécessairement une influence de long terme sur le profit de l'entreprise qui le réalise? Peut-elle s'engager de manière crédible pour dissuader un entrant potentiel d'entrer sur son marché ? Le niveau d'investissement stratégique est-il plus élevé ou moins élevé que celui qui aurait prévalu en l'absence de toute considération stratégique par rapport à l'entrée? Autrement dit, dans quelles circonstances un investissement stratégique d'une firme en place conduit à un surinvestissement et dans quelles autres à un sous-investissement, la situation de référence étant le niveau d'investissement non stratégique. Ce sont à ces questions que tente de répondre l'article de Drew Fudenberg et Jean Tirole (1984) en prolongeant les travaux antérieurs de Michael Spence $(1977,1979)$ et Avinash Dixit $(1980)$.

Pour y voir plus clair, considérons à nouveau un jeu à deux étapes dans lequel une firme en place choisit à la première étape un niveau d'investissement portant sur un actif de nature irréversible. A la deuxième étape, l'entrant potentiel choisit soit de ne pas entrer, soit d'entrer, sachant que dans ce dernier cas, une concurrence de court terme s'instaure entre la firme installée et la firme entrante. Pour chaque valeur de l'investissement de la première étape, la firme en place anticipe l'équilibre de Nash de la concurrence qui s'instaure durant la deuxième étape. Dans une logique à rebours, la firme en place choisit alors son niveau d'investissement, selon qu'il est plus profitable pour elle de dissuader l'entrée (cas noté D) ou de s'en accommoder (cas noté A).

Avant d'entrer plus en détail dans ce type d'analyse, donnons une intuition du type de raisonnement utilisé. Prenons l'exemple d'un investissement qui réduit le coût marginal de production d'une entreprise en place. Un tel investissement peut être judicieux s'il signale la capacité de l'entreprise en place d'adopter une attitude suffisamment agressive pour dissuader un concurrent potentiel d'entrer sur le marché. Mais si cet investissement ne parvient pas à dissuader l'entrée, la question de son intérêt se pose. En fait, si l'entrée a lieu, et que la concurrence sur le marché se déroule en quantités (concurrence à la Cournot), l'investissement de réduction du coût marginal garde sa valeur stratégique car il signale à l'entrant la capacité de combat de l'entreprise en place, ce qui a pour effet de réduire les ambitions de l'entrant. Mais si l'entrée a lieu, et que la concurrence sur le marché se déroule en prix (concurrence à la Bertrand), l'investissement de réduction du coût marginal est contreproductif car il accroît l'intensité de la concurrence, ce que ne désire pas nécessairement l'entreprise en place. Dans ce cas, celle-ci aurait plutôt intérêt à paraitre plus conciliante à l'égard de son nouveau concurrent, en adoptant un profil plus bienveillant. Il est important de comprendre les raisons de ces différences afin d'éviter en tout état de cause la solution simpliste qui consisterait à ce que les opérateurs sur un marché se contentent d'imiter ce qui a donné de bons résultats sur d'autres marchés. Cela permettrait également d'éviter aux autorités de la concurrence d'édicter des règles universelles, sans tenir compte des spécificités du marché en examen.

Drew Fudenberg et Jean Tirole (1984) ont montré que deux facteurs sont essentiels : la nature de l'investissement stratégique initial et la nature de la concurrence ex post sur le marché. Leur combinaison conduit à une typologie que d'aucuns considèrent comme constituant une contribution importante à ce que devrait être une théorie des comportements d'entreprises en concurrence imparfaite (Carl Shapiro, 1989). Le premier facteur porte sur la nature de l'investissement de la firme en place. Deux possibilités existent. La première correspond à une situation où un accroissement du niveau d'investissement, par rapport à ce qui prévaudrait en l'absence de considérations stratégiques, rend la firme en place plus Agressive (Ag). La seconde possibilité correspond à une situation où l'accroissement du niveau $\mathrm{d}^{\prime}$ 'investissement rend au contraire la firme en place plus Bienveillante (Bi). La nature et le montant de l'investissement signalent ainsi, soit que l'entreprise en place est apte à soutenir une guerre économique rude avec l'entrant, soit que la volonté initiale de l'entreprise en place est d'atténuer la concurrence avec l'entrant.

Le deuxième facteur dont dépend la typologie porte sur la nature des variables par lesquelles s'exerce la concurrence de la deuxième étape du jeu, au cas où le concurrent potentiel serait entré sur le marché. II peut s'agir, soit de variables stratégiquement complémentaires, c'està-dire conduisant à des fonctions de réaction croissantes, soit de variables stratégiquement substituables, c'est-à-dire conduisant à des fonctions de réaction décroissantes. Rappelons qu'une concurrence en prix à la Bertrand est du premier type tandis qu'une concurrence en quantités à la Cournot est du deuxième type. Enfin, en ne traitant pas ici la question de savoir s'il est plus profitable pour la firme en place de dissuader l'entrée (cas noté D) ou de permettre l'entrée en s'en accommodant (cas noté A), il s'agit d'analyser les EPSJ dans les deux cas. Selon la nature des deux facteurs précédents, Fudenberg et Tirole distinguent ainsi quatre types de stratégies, avec des appellations animalières qui se veulent suggestives:

- Une stratégie de type top dog ("chien de garde") consiste à choisir un niveau d'investissement plus élevé que celui en l'absence de considérations stratégiques pour signaler que son auteur adoptera un comportement agressif. Lorsque la concurrence est en substituts stratégiques (quantités), un tel investissement signale en effet à l'entrant potentiel une capacité de guerre économique, ce qui devrait inciter ce dernier à être prudent tant en ce qui concerne la décision d'entrer sur le marché qu'en ce qui concerne celle du niveau de production en cas d'entrée.

Une stratégie de type puppy dog ("chiot de salon") consiste à choisir un niveau d'investissement plus faible que celui en l'absence de considérations stratégiques pour signaler que son auteur adoptera un comportement conciliant. Lorsque la concurrence est en compléments stratégiques (prix), un tel choix signale à l'entrant une volonté de comportement pacifique et invite celui-ci à adopter un comportement non offensif dans la concurrence en prix.

Une stratégie de type fat cat ("gros chat") consiste à choisir un niveau d'investissement plus élevé que celui en l'absence de considérations stratégiques, mais pour signaler cette fois un comportement bienveillant. Lorsque la concurrence est en compléments stratégiques, un tel investissement signale à l'entrant que la firme en place a des intentions pacifiques et invite de ce fait l'entrant à adopter lui-même un comportement pacifique. 
- $\quad$ Une stratégie de type lean and hungry look ("malingre et affamé") consiste à choisir un niveau d'investissement plus faible que celui en l'absence de considérations stratégiques pour signaler que la firme en place prend l'apparence d'un félin malingre mais prompt à bondir, en cas de besoin, pour adopter un comportement agressif. C'est notamment le cas si la nature de cet investissement rend la firme bienveillante à l'égard de sa concurrente et si la concurrence est en substituts stratégiques. Cela implique que la firme en place désire montrer qu'elle est moins bienveillante qu'elle n'en a l'air.

Au total, la typologie des comportements stratégiques, aussi bien pour dissuader l'entrée (cas D), que pour s'en accommoder, selon que le profit de dissuasion est plus élevé (cas D) ou plus faible (cas A) que celui de l'accommodement (cas A), est représentée dans les figures 1 et 2 . L'intuition de cette typologie est finalement très simple. Supposons que la firme en place songe à un investissement qui la rende plus agressive. Si le niveau d'investissement qui annule le profit de l'entrant conduit à un profit de la firme en place plus élevé que celui qu'elle pourrait s'assurer en permettant à son concurrent d'entrer, elle adopte un niveau d'investissement élevé, quel que soit le type de concurrence ex post. C'est une stratégie de type Top Dog. Par contre, si ce niveau d'investissement a un coût trop élevé et qu'il est plus rentable pour la firme en place de permettre l'entrée, elle signalera à son concurrent son intention de renoncer à un comportement agressif en réduisant ce niveau d'investissement en cas de concurrence en prix (stratégie Puppy Dog) et, en l'accroissant au contraire en cas de concurrence en quantités (Top Dog). Les intuitions dans le cas où la firme en place songe à un investissement qui la rend plus bienveillante se déduisent de manière analogue.

Cette typologie est intéressante car elle s'applique à de très nombreuses situations qui illustrent comment une entreprise en place peut tirer avantage de sa position de premier intervenant, soit pour dissuader un concurrent potentiel d'entrer, soit pour mieux tirer parti de l'équilibre qui résulte de l'entrée effective du concurrent.

\begin{tabular}{|c|c|c|}
\hline D & $\begin{array}{c}\text { Investissement } \\
\text { rend firme } \\
\text { Agressive }\end{array}$ & $\begin{array}{c}\text { Investissement } \\
\text { rend firme } \\
\text { Bienveillante }\end{array}$ \\
\hline $\begin{array}{l}\text { Compléments } \\
\text { stratégiques }\end{array}$ & Top Dog & $\begin{array}{c}\text { Lean and } \\
\text { Hungry Look }\end{array}$ \\
\hline $\begin{array}{c}\text { Substituts } \\
\text { stratégiques }\end{array}$ & Top Dog & $\begin{array}{c}\text { Lean and } \\
\text { Hungry Look }\end{array}$ \\
\hline
\end{tabular}

Fig. 1 Typologie des investissements stratégiques en cas de dissuasion de l'entrée (D)

\begin{tabular}{|c|c|c|}
\hline \multicolumn{1}{|c|}{ A } & $\begin{array}{c}\text { Investissement } \\
\text { rend firme } \\
\text { Agressive }\end{array}$ & $\begin{array}{c}\text { Investissement } \\
\text { rend firme } \\
\text { Bienveillante }\end{array}$ \\
\hline $\begin{array}{l}\text { Compléments } \\
\text { stratégiques }\end{array}$ & Puppy Dog & Fat Cat \\
\hline $\begin{array}{l}\text { Substituts } \\
\text { stratégiques }\end{array}$ & Top Dog & $\begin{array}{c}\text { Lean and } \\
\text { Hungry Look }\end{array}$ \\
\hline
\end{tabular}

Fig. 2 Typologie des investissements stratégiques en cas d'accommodation de l'entrée (A) 


\section{c/ Applications}

1. Investissement en capacité de production et concurrence en quantités. C'est la situation analysée par Michael Spence (1977) et Avinash Dixit (1980). Accroître sa capacité de production permet à la firme installée, soit de dissuader l'entrée d'un concurrent (D), soit de se prémunir à l'encontre d'une entrée à grande échelle de son concurrent, car une telle entrée fait chuter le prix du marché et est donc nuisible à l'entrant. II s'agit bien d'une stratégie de type Top Dog.

2. Investissement en publicité et concurrence en prix (Drew Fudenberg et Jean Tirole, 1984). Une entreprise qui informe en premier ses clients potentiels via des messages publicitaires peut espérer capter une partie de ses clients à une période ultérieure, indépendamment des efforts ultérieurs d'un concurrent potentiel. La partie de la clientèle restée fidèle représente alors un actif intangible qui assoit la réputation de la première firme. Un accroissement de cet actif rend la firme en place moins sensible à la concurrence en prix que l'entrant peut exercer, ce qui implique que l'actif constitue un signal pour que la concurrence en prix de la seconde période soit moins vive. Il s'agit bien d'une stratégie de type Fat Cat.

3. Ventes liées et concurrence en prix. Michael Whinston (1990) s'est posé la question de savoir si un monopole sur un marché a les moyens de transposer le pouvoir économique sur son marché d'origine vers un second marché en liant les ventes des produits des deux marchés. Une stratégie de type Puppy Dog consisterait à ne pas pratiquer de ventes liées, mais le caractère stratégique des ventes liées réapparaît si le monopole a les moyens de réduire son coût sur son marché originel. Dans ce cas, il s'agirait d'une stratégie de type Top Dog.

4. Capital productif et concurrence en $R \& D$ (Drew Fudenberg et Jean Tirole, 1984). La situation est celle d'une entreprise en place qui choisit en première période un niveau de capital productif, de sorte que se trouve déterminé son coût de production à la période suivante. L'entreprise en place et un entrant cherchent ensuite à améliorer la technologie de manière à parvenir à une réduction de coût (innovation de procédé), sachant que la concurrence en R\&D pour y parvenir prend la forme d'une course à l'innovation où seul le vainqueur obtient le gain de l'innovation. Le choix optimal du niveau de capital productif de première période conduit à une stratégie de type Lean and Hungry Look.

5. Effet d'expérience et concurrence en prix (Jean Tirole, 1988, Drew Fudenberg et Jean Tirole, 1984). Différents travaux, avant Tirole, avaient montré que l'effet d'expérience ("learning by doing") pouvait être utilisé à des fins stratégiques. Mais, il revenait à Drew Fudenberg et Jean Tirole (1984) de montrer que l'influence de l'effet d'expérience dépend du type de concurrence. Avec une concurrence en quantités, on comprend sans peine qu'accroitre la quantité produite à la première période pour abaisser le coût de production, contribue à accroître la quantité de seconde période, ce qui nuit au concurrent lorsque les variables sont des substituts stratégiques. Dans ce cas, la stratégie optimale de première période est d'accroître l'effet d'expérience, c'est-à-dire d'adopter une stratégie de type Top Dog. Mais estce également une stratégie optimale pour une concurrence en prix ? Elle l'est sans conteste dans le cas où la firme en place choisit de dissuader l'entrée de son concurrent (D), et ce pour les mêmes raisons qui viennent d'être explicitées. Mais dans le cas où la firme en place permet l'entrée $(A)$ et que la concurrence se fait en prix, une stratégie de type Top Dog, consistant à accroître la quantité produite à la première période pour bénéficier au mieux de l'effet d'expérience, serait nuisible pour la firme en place, car elle conduirait à des niveaux de prix trop faibles en deuxième période. Une stratégie de type Puppy Dog est préférable.

Sans avoir épuisé l'ensemble des situations où la typologie de Drew Fudenberg et Jean Tirole (1984) s'applique, on perçoit bien la richesse d'une telle typologie. D'une part, elle rompt avec l'idée qu'un résultat unique puisse prévaloir dans tous les cas de figure. D'autre part, elle structure notre compréhension des comportements stratégiques pouvant être adoptés selon les différentes circonstances.

\section{II.4 Modèles dynamiques d'oligopole}

\section{a/ Quels instruments pour la dynamique industrielle?}

Jusqu'ici nous avons passé en revue des travaux où la concurrence était de court terme (deuxième étape du jeu). Or, ne serait-ce que par souci de réalisme, un modèle dynamique de concurrence est toujours préférable à un modèle statique. Un article pionnier de David Kreps et Michael Spence (1984) avait déjà abondamment illustré pourquoi les comportements présents et les performances d'une industrie dépendent en général de l'histoire de cette industrie. Sur le plan empirique, différentes contributions avaient perçu l'importance de l'effet de long terme des structures industrielles sur la dynamique des prix, sans véritablement parvenir à le modéliser (David Encaoua, 1983, David Encaoua et Paul Geroski, 1986, David Encaoua et Philippe Michel, 1986). Bien avant même la représentation de la dynamique concurrentielle par la théorie des jeux répétés, la notion de collusion tacite, qui remonte à Edward Chamberlin (1933), suggérait déjà que les membres d'un oligopole, conscients de leur interdépendance, sont incités à tenir compte au cours du temps des réactions de leurs concurrents, ce qui peut expliquer qu'ils parviennent à des résultats collusifs, sans nécessairement avoir recours à un contrat explicite en ce sens, comme le soulignera plus tard Louis Kaplow, 2013 (voir le compte rendu de David Encaoua, 2014). Mais les jeux infiniment répétés ne se sont pas avérés comme étant l'outil de théorie des jeux le plus adéquat pour rendre compte de la dynamique concurrentielle. La multiplicité des équilibres des jeux répétés, qui subsiste même lorsque la notion d'équilibre de Nash est affinée par la notion "d'équilibre parfait en sous jeux" de Reinhard Selten, est due au fait que les choix d'une période dépendent de toute l'histoire du jeu, donnant naissance à des phénomènes de type "bootstrap", c'est-à-dire des équilibres se fondant sur une mémoire et un niveau de coordination totalement disproportionnés par rapport aux capacités cognitives des acteurs, au point qu'ils perdent toute signification réaliste. Cela explique un certain mécontentement à l'égard de la théorie des jeux répétés pour représenter la dynamique d'un oligopole.

\section{b/ Concurrence dynamique en quantités.}

Une voie de recherche explorée par Eric Maskin et Jean Tirole (1988a, 1988b), a consisté à garder un cadre temporel infini pour la représentation de la concurrence mais en restreignant l'espace des stratégies. Plus précisément, la restriction a consisté à imposer qu'à chaque période les décisions d'un joueur ne portent que sur les variables qui affectent la fonction de gain du joueur durant la période en question. Cette restriction conduit à des stratégies dites "Markoviennes" (Eric Maskin et Jean Tirole, 1991). Pour avoir une idée du cadre d'analyse, considérons comme le font les auteurs, deux entreprises en situation de concurrence répétée au cours du temps (variable discrète) et supposons en premier lieu que la concurrence porte sur les choix de quantités. Pour simplifier, supposons que le joueur 1 choisisse une quantité durant les périodes impaires et conserve ce choix à la période suivante. De même, le joueur 2 choisit une quantité durant les périodes paires et conserve ce choix à la période suivante. A une date $t^{*}$, le joueur i cherche à maximiser son profit actualisé à

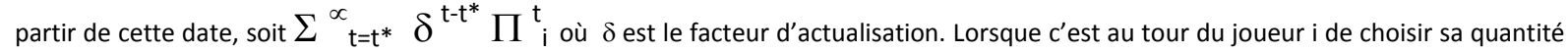
$q_{i}^{t}$, la quantité $q_{j}^{t}$ de son concurrent $j$ a été fixée au tour précédent $\left(q_{j}^{t}=q^{t-1}{ }_{j}\right)$ de sorte que le profit de l'entreprise i à la date $t s^{\prime}$ écrit $\prod_{i}^{\mathrm{t}}\left(\mathrm{q}^{\mathrm{t}}{ }_{i}, \mathrm{q}^{\mathrm{t}-1}{ }_{\mathrm{j}}\right)$. Dans un équilibre stationnaire, la restriction à des stratégies Markoviennes revient alors à dire que le joueur i choisit $\mathrm{q}^{\mathrm{t}}{ }_{\mathrm{i}}$ en fonction de $q_{j}^{t-1}$. Cela revient donc à dire que la stratégie porte sur le choix d'une fonction de réaction $R_{i}$ telle que $q_{i}^{t}=R_{i}\left(q^{t-1}\right)$. 
Ce sont donc les fonctions de réaction elles mêmes qui deviennent des stratégies. Un Equilibre Markovien Parfait (EMP) est alors défini par un couple de fonctions de réactions qui forment un équilibre parfait en sous jeux. Eric Maskin et Jean Tirole (1988a) ont montré que dans un jeu de concurrence en quantités, l'Equilibre Markovien Parfait symétrique existe et est unique. Pour le caractériser, les auteurs vont plus loin, comme l'illustre le paragraphe suivant.

\section{c/ Dissuasion d'entrée dans un cadre de concurrence répétée en quantités.}

En supposant que l'entrée d'une entreprise requiert un coût fixe d'entrée dont la valeur est suffisamment élevée pour qu'un duopole ne soit pas viable, alors qu'un monopole l'est, l'EMP précédent permet de dégager un seuil critique q ${ }^{+}$tel que la production par une firme $\mathrm{d}^{\prime}$ une quantité supérieure à $q^{+}$dissuade le concurrent potentiel d'entrer sur le marché, tandis que la production d'une quantité inférieure $\mathrm{à}^{+}$conduit à ce que le concurrent entre avec une certaine probabilité. Le seuil critique $\mathrm{q}^{+}$joue alors le rôle de quantité de dissuasion de I'entrée. De plus, Eric Maskin et Jean Tirole (1988a) montrent que lorsque la préférence pour le futur est suffisamment élevée, la valeur de $\mathrm{q}^{+}$est supérieure à la quantité de monopole, ce qui implique que la stratégie de dissuasion d'entrée a bien un coût pour l'entreprise qui la pratique. A la limite, lorsque le facteur d'actualisation $\delta$ tend vers 1 , la quantité seuil $q^{+}$tend vers le niveau qu'on obtiendrait en concurrence parfaite.

Cette modélisation généralise donc à un nombre infini d'étapes plusieurs types de modèles. En premier lieu, les modèles antérieurs d'engagement stratégique pour dissuader l'entrée d'un concurrent potentiel, modèles dont on rappelle qu'ils ne comportaient qu'un nombre fini d'étapes. En second lieu, les modèles dits de "prix limite" qui cherchent à représenter le comportement temporel optimal d'une entreprise en monopole face à une menace permanente d'entrée (David Encaoua et Alexis Jacquemin, 1980). En troisième lieu enfin l'approche dite des marchés contestables développée par William Baumol, John Panzar et Richard Willig (1982), dont on sait qu'elle est sujette à différentes critiques (David Encaoua et Michel Moreaux, 1987).

\section{d/ Concurrence dynamique en prix.}

Dans des situations où la concurrence dynamique porte sur les prix et non plus sur les quantités, le même type d'EMP conduit à des résultats assez différents (Eric Maskin et Jean Tirole, 1988b). D'abord, l'unicité de l'EMP n'est plus assurée. Ensuite, deux types d'équilibre apparaissent, réhabilitant tous les deux des conjectures ou intuitions anciennes qui n'avaient pas reçu jusque là de fondement théorique solide.

Le premier type d'EMP comporte des cycles asymétriques de profils de prix, du type de ceux décrits par Francis Edgeworth (1897). Schématiquement, dans un "cycle d'Edgeworth", une guerre des prix a lieu durant un certain nombre de périodes, suivie ensuite par des phases d'accroissement des prix. Une phase de guerre des prix est caractérisée par le fait qu'une baisse du prix d'une firme induit une baisse de prix du concurrent. Mais au bout d'un certain nombre d'étapes de cette phase, il s'avère que les niveaux de prix atteints sont tellement bas que la poursuite de la phase de guerre des prix devient périlleuse pour chacun des protagonistes. A ce moment, une des deux firmes enclenche une hausse de son propre prix et cette hausse est suivie à son tour par celle de son concurrent. Ce cycle asymétrique se répète au cours du temps, conduisant à des phénomènes qu'on observe sur certains marchés (prix de l'essence par exemple).

Le deuxième type d'EMP rappelle une certaine forme de rigidité des prix que les économistes de la première moitié du $20^{\text {ème }}$ siècle décrivaient sous la dénomination de "prix administrés" (Robert Hall et Charles Hitch, 1939, Paul Sweezy, 1939). Dans ce type d'équilibre, les prix convergent vers un prix focal, la convergence s'opérant selon des fonctions de réaction différentes selon qu'un agent choisit un prix au dessus ou en dessous du prix focal. A une baisse de prix en dessous du prix focal répond une baisse de prix du concurrent. Inversement, une hausse de prix au dessus du niveau focal n'est pas suivie par le concurrent. Cette asymétrie dans les fonctions de réaction rappelle évidemment la célèbre fonction de "demande coudée de l'oligopole" décrite par Robert Hall et Charles Hitch (1939) et Paul Sweezy (1939).

\section{e/ Généralisation et applications.}

Dans cette brève présentation de l'EMP, nous avons conservé l'hypothèse d'Eric Maskin et Jean Tirole (1988a et 1988b) selon laquelle les joueurs prennent leurs décisions de manière alternative au cours du temps. Les mêmes auteurs ont défini de manière plus générale un EMP en s'affranchissant totalement de cette hypothèse (Eric Maskin et Jean Tirole 2001). Tout ceci explique que les travaux d'Eric Maskin et Jean Tirole sur la dynamique industrielle ont eu un impact important. Ils ont d'abord ouvert la voie aux nombreux travaux contemporains de simulation d'équilibres dynamiques décrivant l'évolution des marchés oligopolistiques en intégrant différentes variables d'action (Ariel Pakes et Paul McGuire, 1994, Richard Ericson et Ariel Pakes, 1995, Ulrich Dorazelski et Ariel Pakes, 2007, Ulrich Dorazelski et Mark Satterthwaite, 2010). Ils ont également donné naissance à différents travaux empiriques. Citons notamment ceux qui ont cherché à mettre en évidence des cycles de prix d'Edgeworth sur le marché des stations d'essence aux Etats Unis (Paul Zimmerman, John Yun et Christopher Taylor, 2013, Michael Noel, 2007).

\section{II.5 Critiques et appréciations}

Deux critiques ont été adressées aux travaux qui constituent à présent le socle théorique de l'Economie Industrielle et dont les contributions de Jean Tirole sont parfaitement emblématiques. Premièrement, il a été reproché à ces travaux d'adopter des approches d'équilibre partiel et non d'équilibre général, comme dans la tradition initiée par Léon Walras et poursuivie par Gérard Debreu. La critique consiste à dire que ces travaux perdent de vue un certain nombre de liens structuraux propres à l'équilibre général. Deuxièmement, il a également été reproché le fait que le recours systématique à la théorie des jeux comme instrument d'analyse rend les conclusions de ces travaux tributaires de l'extrême sensibilité des résultats aux hypothèses de modélisation (type d'information, séquentialité des décisions, etc.). Examinons tour à tour ces deux types de critiques.

\section{a/ Approches de la concurrence oligopolistique en équilibre partiel.}

Les approches d'équilibre partiel utilisées pour analyser différentes modalités de la concurrence oligopolistique adoptent comme prémisse de base le fait que l'analyse du fonctionnement d'un marché de concurrence imparfaite puisse être menée indépendamment de ce qui se passe sur d'autres marchés. Ce qui implique par exemple que le fonctionnement des marchés de biens et services soit indépendant de l'emploi. De manière plus rugueuse encore, cette critique revient souvent à poser la question de savoir si un renforcement de la concurrence, bénéfique aux citoyens en tant que consommateurs ne serait pas également nuisible aux citoyens en tant qu'offreurs de travail dans une optique d'équilibre général ? C'est là une remarque qu'on entend souvent, notamment en France, au travers d'une critique radicale des politiques de la concurrence. Trois éléments d'appréciation peuvent être avancés pour répondre à cette critique. Le premier est que l'effet revenu n'est jamais absent des analyses de concurrence oligopolistique en équilibre partiel. Même si les variables de la fonction de demande qui interviennent le plus fréquemment dans ces analyses portent sur les prix, sur les quantités produites, sur les 
capacités de production ou encore sur les dépenses de recherche, il n'en reste pas moins vrai que l'effet revenu est toujours présent par le biais du niveau de la demande. Cet effet n'est simplement pas mis en avant lorsque l'accent porte sur les effets de telle ou telle modalité de la concurrence. Au fond, les modèles d'équilibre partiel procèdent selon la clause "ceteris paribus", tout en sachant que dans tous les modèles d'équilibre partiel, il est possible d'examiner l'effet d'une variation du niveau de la demande due par exemple à une variation de revenu. Cette critique n'est toutefois pas totalement dénuée de sens, dans la mesure où l'influence directe de l'intensité de la concurrence sur le niveau de la demande n'est pas directement prise en compte dans les analyses d'équilibre partiel.

Le deuxième argument est que les substitutions et complémentarités entre les biens de différents marchés provenant des préférences des consommateurs joueraient un rôle plus important dans les modèles d'équilibre général que dans les modèles d'équilibre partiel. L'argument n'est pas recevable du fait notamment des différentes formes de différenciation des produits, même si nous ne disposons pas encore d'une "théorie qui permette d'appréhender de façon synthétique la dynamique de la concurrence, qui mêle inextricablement les dimensions du prix, de la différenciation de produits, de la construction de capacités et de l'innovation de produits ou de procédés et la logique de l'entrée" (David Encaoua et Roger Guesnerie, 2006, p. 49).

Le troisième argument enfin plaide en faveur des approches d'équilibre partiel, en dépit des critiques précédentes. En effet, si la notion d'équilibre général a pu être correctement appréhendée lorsque les marchés sont de concurrence parfaite, c'est loin d'être le cas lorsque les marchés sont de concurrence imparfaite. On a par exemple avancé que le recours au concept d'équilibre général en concurrence parfaite est totalement justifié par le théorème de Gérard Debreu et Herbert Scarf (1967), selon lequel les seules allocations qui subsistent dans le cœur d'une économie concurrentielle, quelque soit le nombre d'agents de cette économie, sont les allocations de l'équilibre concurrentiel. Cela justifie pleinement le recours à l'équilibre général de concurrence parfaite. Mais aucun résultat de cette nature ne subsiste en concurrence imparfaite, ne serait-ce que parce que cette notion se définit par négation de l'une ou l'autre des propriétés définissant la concurrence parfaite, ce qui ouvre la voie à un très grand nombre de modalités d'imperfection de la concurrence (Jean Gabszewicz, 1994). Cet argument revient donc à dire que ce sont finalement les approches d'équilibre partiel qui prennent le mieux en compte les modalités d'imperfection. Le pont entre Antoine Augustin Cournot (1838) et Léon Walras (1883) n'est pas près d'être franchi !

\section{b/ Adéquation de la théorie des jeux pour analyser la concurrence oligopolistique}

Comme on l'a rappelé à plusieurs reprises, les conclusions des modèles de concurrence oligopolistique sont tributaires de l'instrument de théorie des jeux qui permet d'y parvenir. Autant dire qu'il paraît difficile pour ne pas dire impossible de parvenir à des résultats robustes, tant la théorie des jeux est sensible aux détails de modélisation. Est-ce pour autant une critique fondamentale ? Ce point a fait l'objet d'un article de Franklin Fisher (1989) auquel a répondu Carl Shapiro (1989). Fisher développe plusieurs arguments critiques exprimant une certaine insatisfaction vis-à-vis des prédictions de la théorie de l'oligopole auxquelles on parvient à l'aide de la théorie des jeux. Premièrement, une théorie de l'oligopole ne peut en aucun cas être réduite à un jeu statique. Le fait que l'équilibre de Cournot soit un équilibre de Nash du jeu statique de concurrence en quantités ne suffit absolument pas à valider "l'équilibre en quantités à la Cournot" comme solution de l'oligopole. Il en est de même de "l'équilibre en prix à la Bertrand". De manière générale une "solution" du problème de l'oligopole ne saurait être trouvée en se focalisant sur une approche exclusivement statique et, c'est là une exigence que satisfont pleinement les travaux de Jean Tirole que nous venons de survoler.

Deuxièmement, travailler à partir de la forme normale d'un jeu de concurrence ne peut constituer une voie de recherche fructueuse pour la théorie de l'oligopole. La forme extensive du jeu, qui révèle à la fois la séquentialité des décisions et les informations disponibles de chaque joueur, est en tout état de cause, préférable à la forme normale ${ }^{6}$. Si le recours à une représentation sous forme normale reste néanmoins encore fréquent dans les modèles de concurrence oligopolistique, cela s'explique selon Franklin Fisher, par le fait que la forme normale est plus facilement manipulable sur le plan mathématique que ne l'est la forme extensive, ce qui permet de parvenir à des résultats plus sophistiqués ${ }^{7}$ (d'où le titre de la contribution de Fisher: "Games Economists Play : A Noncooperative View"). Là encore, les travaux de Jean Tirole satisfont pleinement cette exigence, en mettant l'accent sur les dimensions séquentielles de la concurrence.

Troisièmement, le recours à la théorie des jeux répétés pour formaliser la persistance de l'interdépendance oligopolistique n'est guère satisfaisante du fait de la multiplicité des équilibres de tels jeux : on sait en effet que toutes les stratégies individuellement rationnelles peuvent constituer un équilibre non coopératif du jeu répété. Cette critique est précisément celle que cherche à éviter l'approche en termes d'Equilibre Markovien Parfait.

Quatrièmement enfin, à défaut d'un résultat unique, une théorie satisfaisante de l'oligopole devrait se contenter de parvenir à un ensemble de prédictions en fonction de l'environnement, pour peu que soit explicitée la manière dont un environnement donné affecte la prédiction d'un équilibre spécifique. L'approche développée par Drew Fudenberg et Jean Tirole (1984) pour analyser la dynamique oligopolistique, que nous avons rappelée plus haut, satisfait explicitement ce dernier argument de Franklin Fisher. Que ce soit pour dissuader un concurrent d'entrer sur un marché ou pour s'accommoder au mieux de son entrée, les comportements qui résultent d'un équilibre parfait-en sous jeux diffèrent selon l'environnement, celui-ci étant spécifié à la fois par la nature de l'investissement stratégique de la firme en place et par la nature de la concurrence. La typologie des comportements stratégiques d'équilibre à laquelle Drew Fudenberg et Jean Tirole sont parvenus devrait ainsi être exempte de la critique de Franklin Fisher.

Par ailleurs, les réponses de Carl Shapiro (1989) aux critiques de Franklin Fisher prolongent cette dernière remarque. Carl Shapiro rappelle d'abord qu'il n'y a jamais eu de solution unique au problème de l'oligopole (comme l'attestent les approches de Cournot, Bertrand, Stackelberg, Edgeworth, Chamberlin) et la non unicité a toutes les chances de perdurer au vu de la diversité des comportements observés sur les marchés oligopolistiques. Reconnaissant que la forme extensive est bien plus riche que la forme normale pour saisir les subtilités de l'interdépendance oligopolistique, Shapiro assigne alors à l'économie industrielle théorique la mission de dégager des typologies pertinentes de comportements stratégiques selon des facteurs d'environnement bien identifiés pour conduire à ce qu'il appelle " $A$ theory of business strategy". On reconnait là bien entendu une marque spécifique des travaux de Jean Tirole avec ses coauteurs Drew Fudenberg et Eric Maskin.

\section{II.6 Relations verticales}

Dans toutes les situations où les producteurs ne vendent pas directement leurs biens aux consommateurs finaux mais passent par des intermédiaires, appelés distributeurs, on observe des pratiques contractuelles diverses telles que l'imposition d'un prix de revente imposé

\footnotetext{
${ }^{6}$ Cet argument est largement partagé par Drew Fudenberg et Jean Tirole (1987): "We believe that the focus on "primitives" implied by the extensive-form approach allows a clearer assessment of the model".

${ }^{7}$ Une citation de Franklin Fisher (1989) résume bien le point: "There is a strong tendency for even the best practitioners to concentrate on the analytically interesting questions rather than on the ones that really matter for the study of real-life industries.
} 
par le producteur au distributeur (RPM), la vente exclusive où le distributeur s'engage à ne vendre sur un territoire donné que les produits du producteur et nullement ceux d'un concurrent, l'attribution de territoires exclusifs où le producteur s'engage auprès du distributeur à ne fournir aucun autre distributeur sur un territoire donné, les ventes groupées où des prix avantageux sont proposés en cas d'achat simultané de plusieurs biens, les ventes liées où des achats séparés de différents biens sont rendus impossibles, etc. Ces pratiques contractuelles sont désignées sous le terme générique de restrictions verticales. Quel(s) traitement(s) doit-on leur réserver lorsque le producteur amont est en position dominante sur son marché ? Autrement dit, ces restrictions verticales peuvent-elles résulter d'un effet de levier du pouvoir de marché du producteur sur le marché amont ou ne sont-elles que des manifestations de la liberté contractuelle entre agents situés à différents stades de la chaîne de production et cherchant à corriger les externalités présentes dans leurs relations ?

Comme on l'a rappelé plus haut, les réponses à ces questions ont longtemps été dominées par les tenants de l'Ecole de Chicago. Leur position revenait à dire que les restrictions verticales ne traduisent nullement l'existence d'un pouvoir de marché amont car le producteur ne peut exploiter ce pouvoir que sur son propre marché, en imposant un prix de vente élevé de son produit. Aux yeux des économistes et juristes de l'Ecole de Chicago, l'existence de telles pratiques signifie simplement que les protagonistes, à savoir l'agent amont et l'agent aval, trouvent là un moyen d'accroître l'efficacité de leurs échanges car ceux-ci sont marqués par la présence d'externalités. Par exemple, un producteur impose à un distributeur la pratique d'une vente exclusive pour simplement l'inciter à exercer un effort commercial approprié. Faut-il de ce fait condamner en soi le principe de la vente exclusive ? Autre exemple, le phénomène bien connu de la double marge dans une chaîne verticale peut être évité par l'utilisation d'un tarif binôme par lequel l'amont incite l'aval à vendre au coût marginal en compensant son manque à gagner par une franchise. Faut-il pour autant condamner le recours à une telle franchise ? Les critiques visà-vis des interdictions per se longtemps pratiquées par les autorités de la concurrence, étaient à la fois virulentes et apparemment justifiées.

Dans une série de deux articles, coécrits l'un avec Patrick Rey (1986), l'autre avec Oliver Hart (1990), Jean Tirole renouvelait profondément le cadre d'analyse des restrictions verticales, ce qui amenait à nuancer sensiblement les positions de l'Ecole de Chicago.

Dans l'article de Patrick Rey et Jean Tirole (1986), l'accent est mis sur l'asymétrie d'information entre un producteur et ses distributeurs. Supposons par exemple que le premier n'ait pas une connaissance précise de la fonction de demande qui s'adresse à chacun des distributeurs. Si le producteur vend son bien à un prix uniforme à tous les distributeurs d'un marché donné, supposé de concurrence parfaite, les distributeurs sur ce marché de concurrence parfaite revendraient le produit au coût marginal, c'est-à-dire au prix auquel ils l'ont acheté au producteur (en supposant que le coût marginal du distributeur est nul, hypothèse dont on peut facilement s'affranchir sans altérer le raisonnement). Le profit du distributeur serait alors nul mais le profit du producteur ne serait pas pour autant maximal, car le prix de vente du produit final ne dépendrait pas de l'état de réalisation de la demande de chaque distributeur. En permettant aux distributeurs de vendre à un prix dépendant de l'état de la demande qui s'adresse à eux, le producteur peut accroître le volume de ses ventes, égal par définition à la somme des ventes des distributeurs. En fait, le problème du producteur provient ici de ce que la concurrence sur le marché de la distribution est parfaite, ce qui empêche chaque distributeur de s'approprier un profit. Une solution possible consisterait à réduire, voire supprimer, cette concurrence, par exemple en attribuant un territoire exclusif à chaque distributeur moyennant le paiement d'une franchise. Ceci inciterait le distributeur à se réapproprier un profit positif en agissant comme un monopole rationnel qui ajuste son prix à l'état de la demande. On retrouve là une justification donnée par l'Ecole de Chicago à l'existence des territoires exclusifs. Une interdiction absolue de cette pratique serait dans ce cas, pour reprendre les termes d'un membre influent de cette Ecole, une manifestation du paradoxe de l'antitrust, à savoir que l'interdiction empêche la réalisation d'états efficaces. En fait, toute la subtilité de l'approche de Patrick Rey et Jean Tirole (1986) est de montrer que la condamnation d'une restriction verticale ne conduit pas nécessairement à un paradoxe. Une restriction verticale qui accorde un pouvoir de marché au distributeur peut en effet être bénéfique au producteur et au distributeur, mais pas nécessairement aux consommateurs. Ceux-ci sont perdants si le prix d'achat du produit final augmente. Il est même possible que le surplus global, somme des profits du producteur, du distributeur et du surplus des consommateurs soit plus faible à la suite de cette pratique. Dans ce cas, on aurait un exemple de restriction verticale, justifiée par les tenants de l'Ecole de Chicago puisqu'allant dans l'intérêt commun des deux protagonistes, mais qui devrait être condamnée par ceux qui considèrent que c'est l'intérêt collectif (le surplus global) qui doit être le critère prioritaire d'une politique de la concurrence.

Rétrospectivement, cette argumentation peut paraître un peu puérile au lecteur averti, mais il faut se souvenir qu'au milieu des années 1980, elle était nécessaire face à une pensée établie qui cherchait à parvenir exactement à la conclusion inverse. L'enseignement le plus général que la politique de la concurrence en a tiré est que si l'interdit de toute restriction verticale est une mesure inappropriée parce qu'excessive, une réhabilitation systématique sur le plan juridique de l'ensemble de ces pratiques est tout autant excessive. En un mot, ce type d'analyse ouvrait la voie à un remplacement progressif des règles d'interdits per se par des règles de raison.

\section{II.7 Impacts sur les politiques de la concurrence}

Même si les contributions de Jean Tirole sont essentiellement de nature théorique, on peut affirmer sans risque de se tromper qu'elles ont eu un profond retentissement en matière de politique de la concurrence. Nous nous contenterons ici de développer deux aspects : i/ celui des principes généraux auxquels obéit maintenant cette politique dans la plupart des économies développées et, ii/ celui plus spécifique lié au traitement de la forclusion.

\section{a/ Principes généraux}

Un des principes généraux dans l'application du droit de la concurrence émergeant des travaux de Jean Tirole est de préférer une approche selon la "règle de raison" à une approche selon la "règle per se". Cela veut dire qu'il est préférable, au plan de l'intérêt collectif, de privilégier le traitement du cas par cas de chaque affaire plutôt que d'appliquer des règles universelles édictant des interdits, dont le non respect constituerait en soi une violation du droit de la concurrence. Cela n'a rien d'étonnant sachant combien l'effet des stratégies d'entreprise est sensible aux détails de leur environnement. Prenons quelques exemples. Dans l'analyse du contrôle des concentrations, que ce soit en France, dans l'Union Européenne ou aux Etats Unis, les autorités de la concurrence séparent maintenant de manière très nette l'analyse des effets unilatéraux de celle des effets coordonnés. Les premiers ne concernent que les effets d'une augmentation des prix sans création d'une position dominante, alors que les seconds s'attardent sur les risques que la création d'une position dominante fait peser sur l'intensité de la concurrence. Mettre l'accent sur les effets unilatéraux nécessite évidemment des analyses assez fines, plus fines en tout cas que celles qui consistent à calculer un indice de concentration et à fixer un seuil de concentration au-delà duquel l'opération est jugée potentiellement nuisible à la concurrence. Un deuxième exemple de la pertinence de la règle de raison concerne le traitement des ventes liées. Jean Tirole (2005) montre que le traitement au cas par cas des ventes liées s'impose car les entreprises qui ont recours à cette pratique peuvent le faire pour une série de raisons distinctes dont l'impact sur le surplus social varie dans un large spectre. Les ventes liées peuvent tout aussi bien servir pour accroître l'efficacité économique que pour éliminer un concurrent. De plus elles peuvent être pratiquées aussi bien par une entreprise disposant déjà d'un pouvoir de marché sur un des deux produits liés que par une entreprise sans 
aucun pouvoir de marché, et donc l'effet de levier supposé être à l'œuvre dans les ventes liées ne doit pas être invoqué de manière systématique. Enfin, ce qui est dit à propos des ventes liées pourrait être répliqué pour l'analyse des comportements dits de prédation. Si tous ces arguments plaident pour le recours à la règle de raison dans l'application du droit de la concurrence, les critiques à l'égard de cette approche ne manquent pas pour autant de s'exprimer, ne serait-ce que parce que ce principe n'offre pas une sécurité juridique suffisante aux entreprises, dans la mesure où lois de l'antitrust doivent être telles que les entreprises puissent raisonnablement anticiper ce qui est licite et ce qui ne l'est pas avant de s'engager dans des comportements qui risquent d'être jugés délictueux ex post. C'est pour cela que certains économistes penchent vers l'emploi de règles de raison structurées, procédures intermédiaires susceptibles de concilier la flexibilité des règles de raison pures et les avantages en termes de prévisibilité et donc de sécurité juridique des règles per se (voir la contribution de David Spector, p. 271-285, dans David Encaoua et Roger Guesnerie, 2006).

\section{b/ Forclusion}

La forclusion est définie de manière générale comme la capacité d'une firme détenant un pouvoir économique sur un marché d’affecter par son comportement la structure d'un autre marché. Elle peut le faire de diverses façons. Par exemple, en étant la seule à produire un bien qui se trouve être un input de production sur un marché concurrentiel, elle affecte profondément ce marché en s'intégrant avec l'une des firmes présentes et en refusant de servir les autres firmes. L'input peut être un brevet ou toute autre facilité essentielle à la production du bien. ${ }^{8}$ Comment interpréter alors le comportement d'un producteur amont, qui après avoir intégré une firme aval, forclos le marché aval en refusant de vendre son input aux concurrents de sa filiale ? Autre exemple, une compagnie de transport aérien peut proposer aux voyageurs un programme de fidélisation leur permettant de bénéficier d'un voyage gratuit au-delà d'un kilométrage effectué au sein de la compagnie. Doit-on considérer qu'il s'agit là d'une pratique condamnable, sous le prétexte qu'elle rend plus coûteux le changement de compagnie ? C'est à des questions de ce type qu'est consacré un travail fort intéressant de Patrick Rey et Jean Tirole (2007) sur le thème de la forclusion. Ces problèmes se posent avec acuité dans les économies de réseaux où le contrôle exclusif d'un goulot d'étranglement par un opérateur spécifique peut empêcher tout autre opérateur d'accéder aux clients du premier opérateur. Dans les télécommunications filaires par exemple, le goulot d'étranglement renvoie à la boucle locale constituée par le fil de cuivre reliant un commutateur à l'ensemble des abonnés situés au voisinage du commutateur.

Sous quelles conditions peut-on estimer qu'un comportement de forclusion est dommageable pour la concurrence ? La question ne peut bien entendu recevoir de réponse unique. II faut d'abord préciser en quel sens elle est dommageable. Elle peut l'être parce qu'elle nuit à la concurrence en soi, celle-ci devant être un objet de droit à protéger. Ou bien, elle peut l'être parce qu'elle nuit aux consommateurs. Les deux conceptions ne sont évidemment pas équivalentes et elles constituent une ligne de tension dans l'application du droit de la concurrence, même si les politiques contemporaines de la concurrence privilégient indéniablement la seconde approche (David Encaoua et Roger Guesnerie, 2006). Par ailleurs, la notion de comportement de forclusion doit elle-même être précisée car elle entre parfois en conflit avec des droits acquis. Par exemple, une entreprise qui détient un brevet dont l'accès est indispensable à la production d'un nouveau bien, est-elle coupable de forclusion lorsqu'elle refuse de licencier son brevet à un concurrent sur le marché du nouveau bien, ou ne fait-elle qu'exercer là son droit de propriété intellectuelle qui lui garantit l'utilisation à sa guise de son titre de propriété ?

En cherchant à répondre à toutes ces questions, Patrick Rey et Jean Tirole se situent en fait par rapport aux positions de l'Ecole de Chicago pour qui une entreprise possédant un pouvoir de marché en amont n'a pas besoin d'exploiter ce pouvoir pour conquérir un autre marché. Ils élaborent pour cela un modèle de base très simple que nous explicitons quelque peu ici pour que le lecteur apprécie la logique du raisonnement utilisé. Ils considèrent un jeu à deux étapes entre une entreprise amont, seule sur son marché, et deux entreprises aval qui distribuent le produit amont. A la première étape, l'entreprise amont propose à chacune des deux firmes un contrat tarifaire non linéaire qui spécifie le montant qu'elle doit payer selon la quantité qu'elle commande à la firme amont. Au vu du contrat tarifaire qui lui est proposé, chaque firme aval commande alors à la firme amont une certaine quantité, ce qui amène la firme amont à produire la quantité totale commandée. A la deuxième étape, les deux firmes aval se font concurrence en prix, sachant qu'elles sont contraintes par les capacités correspondant aux quantités qu'elles ont préalablement commandé à la firme amont. Cette situation de concurrence en prix à capacités données (concurrence à la Bertrand-Edgeworth) peut être simplifiée en supposant que la quantité totale écoulée sur le marché est toujours égale à la quantité totale commandée. Le prix commun est alors celui auquel le marché permet d'écouler la quantité totale commandée selon la fonction de demande. Remarquons bien que dans ce jeu, la firme amont produit la quantité globale qui lui est commandée avant que la demande finale ne s'exprime sur le marché. L'équilibre parfait du jeu à deux étapes dépend alors d'hypothèses informationnelles sur les contrats tarifaires, plus précisément selon que le contrat tarifaire proposé à une firme aval est observé ou non par l'autre firme aval. A partir d'une situation de référence où les deux contrats sont observés par les deux firmes, les auteurs parviennent à restituer la logique d'un comportement de forclusion lorsque les contrats sont secrets.

Commençons par la situation de référence où les contrats sont observables par les deux firmes et non renégociables. Dans ce cas, il s'avère que la firme amont est en capacité de s'approprier le profit de monopole sur le marché amont, sans avoir à exclure l'une quelconque des deux firmes aval. Il suffit pour cela qu'elle propose à chacune des deux firmes aval un contrat tarifaire pour lequel la firme amont obtient la moitié de son profit de monopole. Cela permet bien à la firme amont de s'approprier la totalité de son profit de monopole.

Mais dès qu'on quitte la situation de référence, en abandonnant soit l'hypothèse d'observabilité parfaite des contrats ou/et l'hypothèse de la non renégociation des contrats, la solution de la situation de référence n'est plus crédible. En effet, en cas de contrats tarifaires secrets, étant donné le contrat tarifaire de référence proposé à l'une des deux firmes aval, il n'est plus dans l'intérêt de la firme amont de maintenir le contrat tarifaire de référence auprès de l'autre firme aval. De ce fait, les contrats tarifaires de référence perdent leur crédibilité lorsque les contrats sont secrets. Dans ce cas, les équilibres parfaits du jeu dépendent éminemment des conjectures que fait chaque firme sur le contrat tarifaire proposé à l'autre firme. Comme les auteurs n'apprécient guère la notion d'équilibre avec variations conjecturales, ils s'orientent vers une autre piste. Ils font l'hypothèse que la firme amont traite les deux firmes aval comme constituant deux marchés distincts. Dans ce cas, ils montrent que l'équilibre parfait est unique et conduit la firme amont à ne pouvoir s'approprier que le profit global d'un duopole à la Cournot et non plus le profit de monopole. Ils retrouvent ainsi un résultat obtenu dans Oliver Hart et Jean Tirole (1990). L'impossibilité pour la firme amont de s'engager au préalable sur des contrats tarifaires lorsque ceux-ci ne sont connus que par l'entreprise à laquelle ils sont destinés, empêche la firme amont de réaliser le profit de monopole sur le marché amont, en dépit du fait

\footnotetext{
${ }^{8}$ Une facilité essentielle est un input existant qu'il est très couteux de reproduire et qui est cependant indispensable à la production d'un bien. La notion regroupe ainsi des catégories très variées qui renvoient soit à une infrastructure spécifique (voie ferrée, gare, port, réseau électrique, etc.), soit à un input spécifique produit par une seule firme (brevet, système d'exploitation informatique, système de réservation, etc.). Les litiges concernant l'accès à une facilité essentielle sont multiples, notamment lorsqu'il s'agit d'un actif intangible protégé par un titre de propriété intellectuelle (Claude Crampes, David Encaoua et Abraham Hollander, 2006).
} 
qu'elle est seule sur ce marché. $^{9}$ Dès lors, $s^{\prime}$ impose la logique d'un comportement de forclusion, comme par exemple l'élimination de l'une des deux firmes. Un des arguments majeurs de l'Ecole de Chicago, à savoir l'inutilité d'un comportement de forclusion de la part d'une firme en monopole sur son marché, se trouve ainsi disqualifié. On retrouve là toute la différence avec l'argument doctrinal de l'Ecole de Chicago qui considère a priori qu'un comportement de forclusion de la part d'un monopole n'est absolument pas nécessaire pour que le monopole s'approprie l'intégralité de son profit. Un argument plus rigoureux, fondé sur les concepts de la théorie des jeux, montre qu'il est tout à fait possible qu'un monopole ne puisse s'approprier l'intégralité de son profit en l'absence d'un comportement de forclusion. C'est le cas par exemple lorsqu'il lui est impossible de s'engager vis-à-vis de ses distributeurs. Ceci illustre à nouveau pourquoi il convient d'adopter une règle de raison plutôt qu'une règle per se dans l'application du droit de la concurrence.

\section{R\&D, Brevets, Standards et Plateformes : vers une Economie de l'Innovation}

Après avoir présenté quelques travaux qui ont contribué au renouvellement de l'Economie Industrielle, nous examinons à présent d'autres contributions de Jean Tirole qui nous paraissent offrir d'importantes perspectives pour la construction d'un nouveau champ disciplinaire, celui de l'Economie de l'Innovation. On peut considérer que la distinction entre ces deux disciplines n'est pas justifiée a priori, mais deux arguments au moins plaident pour un point de vue différent. Premièrement, les instruments d'intervention publique que sont, d'une part, les politiques de la concurrence pour contrôler les comportements des entreprises sur les marchés et, d'autre part, les politiques de l'innovation pour favoriser l'émergence et le développement de nouveaux marchés, sont parfois en conflit dans la nouvelle économie, en dépit du fait que ces deux instruments sont souvent présentés comme étant complémentaires (David Encaoua et Abraham Hollander, 2002, Claude Crampes, David Encaoua et Abraham Hollander, 2006, David Encaoua et Thierry Madiès, 2014). Deuxièmement, les développements contemporains de l'économie numérique mettent en avant des comportements nouveaux qu'il convient d'analyser de manière spécifique avant d'envisager de nouvelles perspectives pour l'organisation collective de l'activité économique.

Nous examinons ci-dessous les contributions de Jean Tirole dans quatre domaines respectifs :

la concurrence pour le marché, qui à la différence de la concurrence sur le marché, prend la forme d'une course à l'innovation dont seul le premier arrivé tire un bénéfice (III.1),

les modalités d'utilisation des technologies non propriétaires avec des licences spécifiques (III.2),

la caractérisation des marchés biface qui remettent en question les approches traditionnelles du marché pertinent (III.3),

les rassemblements de brevets (pools) et les modalités de leurs licences dans les standards technologiques (III.4).

\section{III.1 Course à l'innovation}

Dans un marché où seul le premier inventeur obtient une rente substantielle, la concurrence prend le plus souvent la forme d'une course pour être le premier. Les courses au brevet ou à l'innovation en constituent un exemple type puisque le vainqueur est celui qui parvient au résultat le premier en déposant un brevet. ${ }^{10}$ La formalisation d'une telle course a suscité beaucoup de travaux. On distingue d'abord les approches stochastiques et les approches déterministes. Ces dernières prennent la forme d'une concurrence technologique ("nontournament") prolongeant simplement la théorie de l'oligopole en permettant d'endogénéiser les structures de marché (Partha Dasgupta et Joseph Stiglitz, 1980 a et b, John Sutton, 1998, 2007). Selon cette approche, les asymétries entre entreprises résultent de la course, ce qui n'implique pas nécessairement l'élimination des entreprises qui n'ont pas gagné la course. Par contre, dans une course au brevet stochastique, seul un vainqueur émerge, mais tant que la course n'est pas terminée, chaque participant conserve une probabilité positive de gagner à chaque instant. De quoi dépend cette probabilité ? Dans un souci de simplification, les premiers travaux (Glenn Loury, 1979, Tom Lee and Louis Wilde, 1980, Jennifer Reinganum, 1981 et 1982) ont supposé que la probabilité de gagner, conditionnelle au fait que personne n'y est encore parvenu, ne dépend que des efforts instantanés de recherche et non des efforts passés. C'est l'hypothèse dite d'une course sans mémoire. Cette hypothèse simplificatrice a été largement utilisée dans un grand nombre de travaux, notamment dans les premiers modèles de croissance endogène (Philippe Aghion et Peter Howitt, 1992). Elle laisse cependant de côté une question importante : Un participant à la course qui se trouverait être en retard par rapport à son concurrent à un moment donné a-t-il la possibilité de le dépasser ultérieurement ? C'est une question importante mais difficile à laquelle Drew Fudenberg, Richard Gilbert, Joseph Stiglitz et Jean Tirole (1983, FGST dans la suite) ont apporté une réponse très élégante. La première distinction qu'ils introduisent est celle entre une course sans mémoire et une course avec mémoire. Comme on vient de le rappeler, dans une course sans mémoire, la probabilité de gagner ne dépend que des efforts présents de chaque participant et non des efforts passés. Autrement dit, l'expérience cumulée due par exemple au montant des dépenses de R\&D engagées dans le passé, n'affecte pas la probabilité d'être le vainqueur. ${ }^{11}$ Dans une course sans mémoire, les deux seules questions pertinentes sont celle de la date à laquelle le vainqueur l'emporte et celle de l'intensité de la concurrence en R\&D que ces courses suscitent. Un des principaux résultats des courses sans mémoire concerne le surinvestissement qu'une telle course génère : dans la mesure où la probabilité de gagner ne dépend pas du passé, la concurrence à tout instant reste très vive, au point d'engendrer une dissipation totale de la rente du vainqueur. Les participants se maintiennent dans la course et se livrent à une surenchère jusqu'à ce que l'un d'eux triomphe. C'est un résultat général : lorsque la concurrence pour le marché est très rude, le niveau de dépenses de $R \& D$ du vainqueur d'une course sans mémoire est tel que son profit actualisé est égal à la dépense encourue, ce qui traduit une dissipation totale de la rente du vainqueur.

FGST (1983) a été l'un des premiers travaux à envisager une course au brevet avec mémoire en adoptant un cadre d'analyse de jeu dynamique. Dans une course avec mémoire, une des questions qui se posent est de savoir s'il est possible qu'un joueur ayant à un moment donné un certain retard d'expérience par rapport à ses concurrents (dû par exemple au fait qu'il est entré plus tardivement dans

\footnotetext{
${ }^{9}$ On retrouve là un résultat analogue à la conjecture de Coase (1972) sur le prix que fixe un monopole pour la vente d'un bien durable. Si le monopole pouvait s'engager sur les prix futurs, il pourrait obtenir le profit de monopole en s'engageant à ne jamais vendre en dessous du prix de monopole. Mais il ne peut s'engager à cela car ce n'est pas crédible. En effet, après avoir vendu la quantité de monopole aux consommateurs de la première période, il est plus rentable pour le monopole de fixer un prix plus bas que le prix de monopole car, ayant déjà servi les consommateurs de première période, la demande à laquelle il est confronté n'est que la demande résiduelle. Mais dès lors, en l'absence d'engagement du monopole sur les prix futurs, les consommateurs de chaque période retardent leurs achats pour bénéficier de prix futurs plus faibles, ce qui conduit inexorablement à éroder le profit du monopoleur.

${ }^{10} \mathrm{C}^{\prime}$ est le principe du first to patent qui prévaut maintenant dans à peu près tous les pays par opposition au first to invent qui a longtemps prévalu aux Etats-Unis.

${ }^{11}$ Le cadre d'une course sans mémoire s'est avéré fructueux car facile à analyser dans la mesure où il ne requiert que des instruments statiques de théorie des jeux.
} 
la course) peut néanmoins rattraper son retard en le compensant par un effort ultérieur accru. La réponse qu'apporte FGST est plutôt positive, mais ce n'est pas un oui universel car, encore une fois, tout dépend de l'environnement.

a/ Dans une course stochastique au brevet avec mémoire et information parfaite, où les dépenses passées sont observées à tout instant, aucun rattrapage n'est possible à l'équilibre du jeu. Si un agent acquiert un leadership à un moment donné, il le conserve tout le temps. Ce résultat est d'autant plus surprenant que dans une course au brevet de nature stochastique, chaque participant garde par définition une probabilité strictement positive de gagner la course. Mais ici comme l'information sur le passé est parfaite et que le jeu est dynamique, un meneur peut toujours adapter son effort de manière à garder le leadership. De façon générale, si un agent est le premier à entrer dans une course au brevet stochastique, avec mémoire et information parfaite, aucun autre agent n'a intérêt à y participer. Cette situation est désignée par FGST par le vocable " $\varepsilon$-préemption", pour souligner que tout avantage initial, aussi petit soit-il, suffit à assurer la victoire. De plus, c'est une victoire très particulière dans la mesure où tout agent suiveur est dissuadé d'entrer dans la course. De ce fait, une course stochastique avec mémoire où l'information est parfaite ne conduit à aucune concurrence durable dans le temps. Notons que ce même résultat d' d'ع-préemption peut prévaloir dans une course au brevet déterministe (Partha Dasgupta et Joseph Stiglitz, 1980, Richard Gilbert et David Newbery, 1982), sachant que le vainqueur est simplement celui qui consacre le plus grand effort de R\&D.

b/ II n'en est pas de même dans d'autres situations. Par exemple, dans une course avec mémoire comportant plusieurs étapes, chacune d'elles devant être franchie avant d'aborder la suivante, une firme ayant moins d'expérience peut néanmoins gagner une étape et rattraper son retard. L'idée est simple et peut être illustrée à l'aide d'une course à deux étapes. Comme la première est une course stochastique, il est possible que malgré un certain retard à entrer dans la première étape, une firme entrée plus tardivement, parvienne à franchir la première (par chance) la ligne d'arrivée de la première étape. Elle compense alors son retard initial en étant la première à entamer la seconde étape, accroissant ainsi la possibilité qu'elle soit le vainqueur final.

c/ D’autres situations conduisent également à la possibilité de compenser un éventuel retard, notamment du fait des spécificités de l'information. Supposons par exemple qu'un participant à la course ne puisse observer la position de son concurrent qu'avec un certain retard. Pour avoir une intuition du mécanisme qui est à l'œuvre, reprenons la lumineuse comparaison que fait Tirole dans son ouvrage de 1988 (p. 398): "Considérons une course à pied entre deux athlètes. Supposons que tout le monde sache que les deux athlètes se valent et qu'ils préfèrent se réserver (courir lentement) plutôt que s'épuiser en courant vite. Supposons aussi que le meneur ait des yeux derrière la tête et puisse contrôler si le suiveur le rattrape. Puisque le meneur peut conserver la tête en accélérant si son rival accélère, le rival n'a aucun intérêt à s'engager dans la course. Le leader peut donc courir lentement sans crainte d'être dépassé. Mais l'histoire change du tout au tout si les deux athlètes courent sur des pistes séparées par un mur. Supposons que le mur ait des trous, de sorte que, de temps en temps, chaque athlète peut faire le point sur sa position relative. Maintenant, le meneur ne peut plus courir lentement; s'il le faisait, le suiveur pourrait courir rapidement, dépasser le leader sans qu'il le remarque et l'obliger à abandonner la course au trou suivant. Donc, des retards dans l'information (ou dans les réactions) créent la concurrence. II en va de même pour les courses au brevet". C'est cette idée qui est formalisée dans FGST (1983).

On retiendra de ce très bref survol des courses stochastiques au brevet trois conclusions. La première est qu'il faut être très prudent à propos des conclusions d'un modèle de course au brevet. Selon l'environnement privilégié, un grand nombre de configurations sont possibles et il parait difficile de conclure à la validité de l'un ou l'autre des résultats, si l'on n'a pas pris soin de préciser l'environnement adopté. En particulier, savoir si un retard temporaire peut ou non être rattrapé est une question délicate vis-à-vis de laquelle nous ne disposons pas d'une réponse unique. Deuxièmement, la question de la possibilité ou de l'impossibilité d'un rattrapage est très concrète quand on s'intéresse aux mécanismes de concurrence pour le marché et non pas sur le marché. Songeons par exemple aux conditions d'émergence d'un standard technologique de facto. Troisièmement, une des idées importantes inaugurée dans FGST et qui sera reprise dans de nombreux travaux ultérieurs est que la concurrence est très intense dans des courses au brevet où les concurrents sont proches les uns des autres (au coude à coude diront plus tard Philippe Aghion, Christopher Harris, Peter Howitt et John Vickers, 2001) mais elle est moins intense lorsque l'un des concurrents possède une avance notable. Enfin, ne perdons pas de vue que pratiquement tous les modèles de croissance endogène ont recours à une modélisation spécifique de course au brevet et les résultats de ces modèles sont de ce fait très contingents à la modélisation choisie (Philippe Aghion et Rachel Griffith, 2005, David Encaoua et David Ulph, 2005, Philippe Aghion, Ufuk Akcigit et Peter Howitt, 2014, Vincenzo Denicolo et Piercarlo Zanchettin, 2012)

\section{III.2 Logiciels libres et licences}

\section{a/ Les systèmes "open source"}

Les systèmes "open source" sont des systèmes non propriétaires, souvent désignés sous le label de "systèmes libres". Certains logiciels (notamment de traitement de texte et d'exploitation tels que Linux ou le serveur internet Apache) figurent en tête de liste de ces systèmes libres, ne serait-ce que parce que la brevetabilité des logiciels n'est pas partout autorisée et, là où elle l'est, elle continue de faire débat. Les logiciels non propriétaires sont des programmes dont les auteurs publient le code source, en permettant aux utilisateurs, à la fois d'y avoir accès moyennant l'obtention d'une licence spécifique, et d'apporter éventuellement des améliorations par rapport aux performances du programme, à condition que ces améliorations soient elles-mêmes diffusées en "open source". Une des questions intrigantes à propos du très important développement des logiciels libres est de comprendre les motivations qui animent leurs auteurs, en nombre de plus en plus élevé par ailleurs. Est-ce du pur altruisme? Mais alors pourquoi n'observe-t-on pas des comportements analogues dans d'autres activités ? Après avoir retracé l'histoire du logiciel libre, Josh Lerner et Jean Tirole (2002) avancent une explication, à partir de l'observation de quelques cas emblématiques (Linux, Apache, Perl et Sendmail). L'explication avancée, ne s'appuie pas sur une analyse théorique spécifique à cette question, mais plutôt sur l'analyse économique d'une tout autre notion, celle des déterminants d'une carrière professionnelle qui s'appuie elle-même sur quelques notions d'économie industrielle.

Commençons par ces dernières. En tout premier lieu, de nombreux travaux soulignent la place croissante qu'occupent aujourd'hui les utilisateurs de technologies dans le processus d'innovation : dans nombre d'activités, les innovateurs sont davantage des utilisateurs plutôt que des producteurs (Eric von Hippel, 1988, 2006, Yochai Benkler, 2006, David Encaoua, 2006). Autrement dit, les améliorations successives d'une innovation sont souvent dues aux utilisateurs plutôt qu'à des innovateurs. Les solutions apportées par certains utilisateurs aux problèmes auxquels ils sont confrontés sont en effet porteuses de progrès importants et ont parfois constitué la source principale du processus d'innovation ultérieur. L'histoire du développement de l'informatique et celle de l'émergence d'Internet (Shane Greenstein, 2010) en sont des illustrations. En second lieu, la collaboration entre chercheurs affiliés au monde académique et chercheurs affiliés aux entreprises a vraisemblablement permis que le principe de la "science ouverte" qui régit les premiers, pénètre progressivement chez les seconds, conduisant à une floraison d'applications en "open source" (Rebecca Henderson et lain Cockburn, 1996). Josh Lerner et Jean Tirole (2002) retracent ainsi différentes phases d'interactions réciproques entre la science et ses applications qui ont 
contribué à des pratiques collaboratives et de partage ayant donné naissance in fine au principe de logiciels libres cohabitant avec des logiciels propriétaires, les deux étant licenciés selon des principes différents. Toutefois, aussi suggestives qu'elles soient, ces explications ne suffisent pas à rendre compte des spécificités de l'open source et les auteurs se tournent alors vers des statistiques de contributions à des logiciels libres. Ils découvrent d'abord que la distribution des contributeurs aux projets d'open source est très fortement asymétrique : la majeure partie (plus de $75 \%$ ) des milliers de contributeurs à des projets d'open source n'ont contribué qu'une seule fois, tandis que moins de $4 \%$ seulement ont contribué à plus de cinq projets. Mais, lorsqu'on comptabilise en pondérant par le nombre de contributions, le premier décile des programmes représente à lui tout seul $72 \%$ de l'ensemble des programmes open source! Cette première indication signale que les principaux contributeurs (ceux qui contribuent plus d'une fois) pourraient avoir des caractéristiques ou des motivations spécifiques. Complétées par d'autres informations, comme par exemple la forte prépondérance de la mention edu dans l'adresse mail de ces développeurs (mention signalant leur appartenance académique), ces statistiques amènent Josh Lerner et Jean Tirole à penser que la population des grands développeurs de logiciels libres est en fait fortement élitiste ! Ces grands développeurs semblent en effet poursuivre une stratégie de signalement, comme le confirment les entretiens avec les développeurs emblématiques mentionnés plus haut.

Se tournant alors vers la littérature économique sur les carrières professionnelles (Bengt Holmström, 1999) qui combine les efforts induits à la fois par des incitations extrinsèques au développement de la carrière professionnelle et par des motivations purement intrinsèques, Josh Lerner et Jean Tirole (2002) en tirent trois prédictions concernant les efforts pour gravir les échelons:

1. Les efforts sont d'autant plus importants que les performances qui en résultent sont visibles par les audiences concernées (supérieurs hiérarchiques, collègues, pairs dans le monde académique, employeurs potentiels, etc.).

2. Les efforts sont d'autant plus importants que leur impact sur les performances est élevé.

3. Les efforts sont d'autant plus importants que les performances observées sont perçues comme étant fortement corrélées au talent de leur auteur.

La partie empirique du travail de Josh Lerner et Jean Tirole, sur laquelle nous ne revenons pas ici, confirme que ces principes sont bien présents dans le travail des programmeurs qui développent des logiciels en open source. Les auteurs avancent ainsi l'idée que développer des logiciels libres (en en livrant donc le code source) est un signal transparent (principe 1), efficace (principe 2) et d'autant plus informatif sur le talent du développeur que la diffusion de son travail est large (principe 3). Offrir un signal crédible à ses pairs ou sur le marché du travail, tout en se faisant plaisir : telle est finalement l'hypothèse avancée par Josh Lerner et Jean Tirole pour expliquer les motivations des développeurs de logiciels libres. Au total, c'est là un remarquable exemple d'article sans équations, ni modèle analytique ou économétrique, mais dont les conclusions parviennent néanmoins à convaincre le lecteur, tant le travail présenté révèle un indéniable talent de réflexion économique, de curiosité intellectuelle et d'érudition pour éclairer une question qui peut tout aussi bien concerner des sociologues ou des psychologues. II n'est pas interdit de penser par ailleurs que ce genre de réflexions n'est pas étranger à la motivation ayant conduit plus tard Jean Tirole, associé à Roland Benabou, à chercher à mieux comprendre les rôles respectifs des incitations extrinsèques (essentiellement pécuniaires) et des motivations intrinsèques (non pécuniaires comme la satisfaction du travail bien fait) dans beaucoup de comportements humains (Roland Benabou et Jean Tirole, 2002, 2003, 2009).

\section{b/ Restrictions dans les licences open source}

Les licences de logiciels libres comportent un certain nombre de restrictions d'usage dont l'une des plus importantes consiste à interdire au licencié de vendre une version améliorée du programme en tant que logiciel propriétaire ne dévoilant pas le code source. Pourquoi, quand et comment ces restrictions apparaissent-elles ? C'est à ces questions qu'est consacré l'article de Josh Lerner et Jean Tirole (2005). En tout premier lieu, le recours à l'histoire du logiciel libre est éclairant. Après que les premiers logiciels non propriétaires aient été licenciés au titre du droit d'auteur, sans aucune restriction d'usage, un certain nombre de comportements contraires à une certaine éthique sont apparus, comme par exemple le fait qu'un utilisateur, à un stade particulier de la chaîne, ait été amené à recourir à des logiciels propriétaires, alors que lui-même avait bénéficié de logiciels libres. En réaction à ce type de déviations, un programmeur du MIT, Richard Stallman, lançait en 1983, dans une pure tradition hacker, le système d'exploitation libre dit GNU (acronyme anglais "Gnu's Not Unix") qui permettait de disposer de tout un ensemble de logiciels utilisables sous un même système d'exploitation, comme cela avait précédemment été le cas pour UNIX. ${ }^{12}$ Ainsi, plutôt que de permettre une libre utilisation des systèmes d'open source en les transférant simplement dans le domaine public, Stallman requérait des utilisateurs qu'ils souscrivent à une licence publique GNU, satisfaisant trois propriétés : i/ Le code source du programme doit être disponible ; ii/ Chaque utilisateur est libre d'apporter les modifications souhaitées au programme du code source; iii/ La commercialisation des modifications du code source est elle-même soumise à deux conditions : a/ Une commercialisation éventuelle doit s'opérer selon une licence GNU ; b/ Un logiciel open source ne doit pas être mélangé avec un logiciel propriétaire. Ces trois propriétés (i/, ii/ et iii/) définissent une licence dite GPL ("General Public License") dont une variante moins restrictive, dite LGPL ("Lesser General Public License") introduisait plus de flexibilité dans la condition 3 b.

Après avoir procédé au rappel historique, Josh Lerner et Jean Tirole (2005) ont construit un modèle théorique prenant en compte ces différentes caractéristiques et cherchant à répondre aux questions de savoir, d'une part, quels licencieurs ont recours à une licence restrictive de type GPL plutôt qu'à une licence commerciale classique n'introduisant aucune restriction d'usage et, d'autre part, quels types d'utilisateurs (licenciés) sont à même d'accepter une telle licence. Nous ne revenons pas ici sur le modèle, ni sur ses résultats, sinon pour souligner le soin extrême des auteurs à d'abord trouver les phénomènes les plus marquants qu'il convenait de privilégier avant de proposer une modélisation spécifique et de soumettre la validité des résultats à différents tests empiriques et économétriques, parvenant de la sorte à un défrichement remarquable d'une question jusque là ignorée, alors même que son intérêt pratique est évident. Plutôt que d'exprimer une préférence marquée a priori pour l'un ou l'autre type de licence, ce que ne manquent pas de faire en général aussi bien les thuriféraires des licences d'open system que ceux des licences propriétaires, Josh Lerner et Jean Tirole ont procédé à une véritable analyse objective des déterminants des licences open system, pour identifier les types de licencieurs et de licenciés pour lesquels les bénéfices des licences open system l'emportent sur leurs coûts.

\section{III.3 Marchés bi-face}

Un marché biface est un marché sur lequel une ou plusieurs plateformes permettent des transactions entre deux catégories d'utilisateurs, en cherchant d'abord à les rassembler, puis à accroitre le volume de transactions par des structures de prix appropriées. En cherchant à comprendre et décrypter les principes qui fondent les relations entre les banques des commerçants et celles des clients détenteurs de cartes bancaires, Jean Charles Rochet et Jean Tirole (2003 et 2006) découvrent qu'un marché biface est un marché sur lequel le volume de

\footnotetext{
${ }^{12}$ Selon Wikipedia (GNU), "au début des années 1990, le projet GNU possède une version utilisable de (presque) tous les éléments nécessaires à la construction d'un système d'exploitation".
} 
transactions entre deux types d'utilisateurs dépend davantage de la structure des prix pratiqués par la plateforme pour les deux catégories d'utilisateurs, que du niveau des prix que pratique la plateforme pour chaque catégorie. Cette importante découverte a plusieurs implications. Premièrement, elle invite à une redéfinition du marché pertinent. La définition d'un marché pertinent dans le cas d'un marché biface doit prendre en compte l'impact d'une variation du tarif payé par un groupe sur les profits réalisés sur l'autre groupe. L'analyse des prix pratiqués sur de tels marchés est donc confrontée à une spécificité très particulière : la structure des prix ne reflète pas forcément celle des coûts, une marge élevée n'est pas forcément synonyme de pouvoir de marché, un prix inférieur au coût marginal n'est pas une preuve de prédation. De ce fait, l'analyse de la structure de marché qui intervient au préalable dans certaines affaires relevant du droit de la concurrence doit tenir compte des éventuelles caractéristiques bifaces des marchés. Deuxièmement, certaines des règles présentes dans le droit de la concurrence lorsqu'il s'agit d'un marché traditionnel ne sont plus valables dans un marché biface. Alors qu'une tarification en dessous du coût marginal est en général prohibée sur un marché traditionnel, il ne saurait en être de même dans un marché biface. Prenons l'exemple d'un journal quotidien auquel sont rattachés des lecteurs d'un côté et des annonceurs de publicité de l'autre côté. Distribuer gratuitement ce journal peut être une stratégie rationnelle et légitime en dépit du fait qu'elle viole la prohibition d'une vente en dessous du coût marginal. La raison d'une telle stratégie dans un marché biface est simple : une distribution gratuite accroit le volume de lecteurs et, de façon conséquente, attire plus d'annonceurs. Le coût du journal peut en fait être financé par les annonceurs, en tenant compte du fait qu'au-delà d'un certain volume d'annonces publicitaires, le nombre de lecteurs peut baisser, du fait de l'attitude publiphobe de certains lecteurs.

Les exemples de marchés biface, où de telles asymétries de prix existent, abondent : cartes de crédit, sites de rencontres, développeurs de jeux vidéo et utilisateurs, lecteurs de journaux et annonceurs de publicité, etc. La justification de telles configurations et la compréhension des modalités de leur fonctionnement sont restées relativement inexplorées jusqu'à un passé récent. Jean Charles Rochet et Jean Tirole (2006) ont en fait ouvert un très large champ d'investigation. Conceptuellement, la théorie des marchés bifaces emprunte à deux théories : celle des externalités de réseaux et celle des activités multi-produits. A la première (Michael Katz et Carl Shapiro, 1985, 1986, Joseph Farrell et Garth Saloner, 1985, 1986), elle emprunte l'idée qu'il existe des externalités indirectes entre les deux types d'agents. L'intérêt des participants d'un côté du marché dépend des caractéristiques de la demande de l'autre côté. Ce sont précisément ces externalités qui justifient l'existence d'une ou plusieurs plates-formes d'intermédiation. A la théorie de la multi-production, elle emprunte l'idée que ce qui importe c'est la structure des prix, celle-ci étant moins affectée par le pouvoir de marché sur chacun des produits, du fait de la demande et des coûts joints. Alors que les analyses de ces deux théories sont restées séparées jusqu'à un passé récent, la théorie des marchés bi-face avancée par Jean Charles Rochet et Jean Tirole $(2003,2006,2008)$ exploite simultanément les deux dimensions.

Les prix comportent en fait deux composantes. La composante fixe, conditionne la présence des deux parties sur la plateforme. La composante variable, qui définit la charge d'usage, détermine l'acceptation des deux parties de réaliser des transactions sur la plateforme, une fois qu'elles y sont. Ces éléments permettent de comprendre la différence de base entre un marché traditionnel (mono face) et un marché biface. Supposons que l'adhésion à une plateforme soit déjà réalisée par les deux bouts du marché. La question pertinente est alors de savoir si les agents utilisent ou non intensivement la plateforme. Jean Charles Rochet et Jean Tirole introduisent la structure des prix d'usage de la plateforme, c'est-à-dire les deux composantes variables payées par les deux catégories d'usagers. Un marché biface est ainsi défini par le fait que si $a^{A}$ et a ${ }^{v}$ sont les charges d'usage respectivement payées par un acheteur et un vendeur au propriétaire de la plateforme, le volume des transactions réalisées dépend de $a^{A}$ pour un prix total $a=a^{A}+a^{V}$ donné, à la différence d'un marché traditionnel mono face où le volume de transactions entre acheteurs et vendeurs ne dépendrait que du prix total a. Dans un marché biface, les deux marchés sont structurellement liés. Pour illustrer, le marché des paiements par carte bancaire reliant les banques émettrices et réceptrices des deux types d'agents que sont respectivement les acheteurs et les vendeurs de biens serait un marché mono-face si les trois conditions suivantes étaient réalisées : i/ les charges d'usage que supportent la banque du vendeur et la banques du détenteur de la carte sont intégralement répercutées par la plateforme sur le vendeur et le détenteur; ii/ le vendeur a la possibilité de discriminer les prix selon que le paiement de la transaction s'effectue par carte bancaire ou en cash; iii/ le vendeur et l'acheteur ne subissent aucun coût de transaction du fait du système dual de paiement (liquidités et carte bancaire). Or aucune de ces conditions n'est satisfaite dans la réalité. Plus précisément, la violation d’une au moins de ces trois conditions justifie qu'il s'agit d'un marché biface. Sur le plan théorique, la distinction entre marché mono face et marché biface est importante à plus d'un titre. Revenons par exemple sur le théorème de Coase (1960) selon lequel des inefficacités dans les échanges entre parties, dues par exemple à des externalités, peuvent être résolues par négociation, pour peu que celle-ci n'induise pas des coûts de transaction. Jean Charles Rochet et Jean Tirole (2006) montrent qu'il n'y a pas équivalence entre les deux propositions suivantes : i/ Le théorème de Coase ne s'applique pas car les deux parties ne peuvent atteindre un résultat efficace en négociant; ii/ Le marché est biface car la structure des prix pratiqués par la plateforme est non neutre. En fait, si le marché est biface, alors le théorème de Coase ne s'applique pas. En d'autres termes, la condition i/ est nécessaire mais non suffisante pour ii/. En effet, même en présence de la propriété de neutralité, le théorème de Coase peut ne pas s'appliquer si les informations privées dont dispose chaque protagoniste sont asymétriques.

Au-delà des charges d'usage, la structure des redevances fixes payées par les deux parties pour se rattacher à la plateforme intervient également dans les marchés biface. Le modèle de Jean Charles Rochet et Jean Tirole (2006) généralise un article des mêmes auteurs (2003) en intégrant à la fois les redevances variables et fixes. Dans un marché biface, le volume d'activité dépend, d'une part, des indices de participation des deux groupes à la plateforme, fonctions eux-mêmes des redevances fixes et, d'autre part, du nombre de transactions réalisées, fonction des charges d'usage.

Par ailleurs, les tarifs mis en place peuvent inclure une tarification différenciée pour chaque groupe dans le cas où l'appartenance à l'un des deux groupes est facilement identifiable. On distingue ainsi :

- une contribution à la participation qui peut être différente selon le groupe (tarification à l'accès, à l'inscription, à l'abonnement) ; - une contribution par transaction variant entre les deux groupes (tarification à l'usage).

Le modèle de Jean Charles Rochet et Jean Tirole examine d'abord le cas d'une unique plateforme. Le fonctionnement de la plateforme nécessite des "modalités de coordination" entre les deux catégories d'acteurs qui peuvent prendre diverses formes, comme par exemple, ne pas faire payer l'accès mais seulement l'usage, subventionner la participation d'un groupe par une contribution fixe négative, ou encore lier la participation à l'offre d'un service payant. Un côté du marché peut être déficitaire (un détenteur d'une carte de crédit n'est pas tarifé selon l'usage qu'il fait de sa carte) tandis que l'autre côté peut être profitable (la plateforme prélève une taxe sur les ventes des vendeurs). Le côté excédentaire finance ainsi le côté déficitaire et la plateforme est globalement bénéficiaire.

Le modèle examine ensuite le cas où plusieurs plateformes sont en concurrence et distingue deux sous cas : celui où l'accès de chaque agent est limité à une seule plateforme ("single homing") et celui où l'accès à plusieurs plateformes est permis ("multi homing").

En présence du single homing, les prix et les profits peuvent être faibles car la concurrence entre les plateformes est forte. Un basculement vers une situation où ne subsiste plus qu'une seule plateforme est alors possible ("tipping") car une plateforme unique peut être plus efficace du fait des externalités de réseaux. 
Dans le cas du multi homing, deux possibilités existent: le multi homing bilatéral où les deux groupes d'agents peuvent utiliser chacun plusieurs plateformes et le multi homing unilatéral où seul un des deux groupes d'agents peut utiliser plusieurs plateformes.

Dans le multi homing bilatéral, une complémentarité entre plateformes est requise pour assurer la compatibilité et on entre là dans les modèles de concurrence entre réseaux. L'économie numérique contemporaine développe fortement le multi homing bilatéral aussi bien du côté des développeurs que de celui des utilisateurs. Les développeurs proposent des applications fonctionnant sur diverses plateformes (smartphone, tablette, etc.) et les utilisateurs peuvent y accéder sans coût, ce qui explique que les externalités de réseaux ne conduisent pas à une aussi forte concentration que celle prévalant dans le single homing.

Dans le multi homing unilatéral, dont un centre commercial représente l'exemple type (les acheteurs ne fréquentent en général qu'un seul centre alors que les produits des producteurs sont présents dans tous les centres commerciaux), la concurrence est intense du côté où l'accès est unique (consommateurs dans le cas des centres commerciaux), ce qui amène les centres commerciaux à proposer à leurs clients une série d'avantages plus ou moins gratuits, comme par exemple le parking ou des prix de l'essence plus faibles que dans les stations ordinaires, etc.

\section{III.4 Pools de brevets et standards technologiques}

\section{a/ Pools de brevets}

Un pool de brevets est un accord entre détenteurs de brevets pour mettre en commun leurs brevets et les licencier sous forme groupée à des tiers ou entre détenteurs. L'histoire de ces arrangements a connu des flux et des reflux, avec aujourd'hui une phase de développement très active. Parmi les pools de brevets les plus connus, citons Motion Picture Experts Group (MPEG 2, 1997, MPEG 4, 1998), Bluetooth (1998), DVD-ROM (1998), DVD-Vidéo (1999), 3G Mobile Communications (2001) et One-Blue (2009). Deux thèses s'affrontent à propos des pools de brevets.

La première présente ces accords comme des réponses appropriées au problème dit du "buisson de brevets" (patent thicket), problème qui est présent dans les situations où l'accès à une nouvelle technologie nécessite de recourir à une multitude de brevets fragmentés et enchevêtrés, ce qui implique pour l'utilisateur de devoir payer plusieurs redevances de licences qui conduisent au total à un prix tellement élevé que le marché de la nouvelle technologie peut ne pas voir le jour. Lorsque ces brevets sont complémentaires, leur regroupement sous la forme d'un pool de brevets donnant naissance à une licence globalisée est alors avantageux pour les utilisateurs, dans la mesure où le regroupement évite l'accumulation des différentes marges prélevées sur des licences isolées. C'est l'argument bien connu du problème dual d'Antoine Augustin Cournot (1838) repris par Carl Shapiro (2001). Chaque détenteur de brevet est assimilé à un monopole contrôlant un input nécessaire à la production d'un nouveau bien, sachant que tous les inputs sont nécessaires. Dans ce cas, un monopole intégré unique, offrant l'ensemble des inputs, est plus avantageux à la fois pour les détenteurs de ces inputs et pour les utilisateurs finaux, qu'une série de monopoles indépendants offrant individuellement chacun des inputs.

La deuxième thèse est beaucoup moins favorable aux pools de brevets. A l'instar des accords commerciaux, les pools de brevets serviraient à réduire la concurrence. C'est la raison pour laquelle le regroupement de brevets substituables est considéré comme un moyen d'éviter la concurrence entre les différents brevets, ce qui nuit évidemment aux utilisateurs. Par ailleurs, un certain nombre de clauses apparaissant dans ces regroupements sont de nature à réduire le processus d'innovation, comme par exemple les licences croisées où les détenteurs de brevets du pool se promettent mutuellement de se rétrocéder des licences correspondant aux améliorations ultérieures de leurs innovations.

Dans un article qui a beaucoup contribué à remettre en question nos connaissances sur cette importante question, Josh Lerner et Jean Tirole (2004) partent d'un premier constat. La distinction entre brevets complémentaires et brevets substituables est à la fois peu opératoire et peu pertinente. Rappelons d'abord les définitions de ces termes. Des brevets sont dits complémentaires s'ils sont collectivement indispensables pour un utilisateur de technologie. Autrement dit, disposer de tous les brevets à l'exception d'un seul d'entre eux ne serait d'aucune utilité pour un utilisateur. On comprend alors que le théorème dual de Cournot s'applique à des pools de brevets parfaitement complémentaires. Des brevets sont dits substituables si un utilisateur peut recourir à des technologies alternatives en faisant varier les ensembles de brevets utilisés. Par exemple, il peut exister deux brevets différents, parfaitement valides et, satisfaisant la même fonctionnalité. On comprend alors que des pools de brevets substituables doivent être empêchés de se former, car la concurrence entre brevets isolés conduit dans ce cas à un résultat plus satisfaisant que celui où les détenteurs décident en commun d'un prix global. Josh Lerner et Jean Tirole utilisent ces définitions pour récuser à la fois le caractère opératoire et le statut conceptuel de la distinction entre complémentarité et substituabilité des brevets. Premièrement, il est difficile pour une autorité de la concurrence de savoir si les brevets d'un pool sont complémentaires ou substituables. Dès lors, une telle autorité ne saurait avoir recours à ce critère pour décider si elle doit autoriser ou non la décision de constitution d'un pool de brevets. Deuxièmement, entre les deux cas extrêmes de parfaite complémentarité et parfaite substituabilité se situent une multiplicité de cas intermédiaires qui paraissent plus proches de la réalité. Des inputs peuvent être complémentaires ou substituables en fonction de leurs prix, car les technologies sont en général relativement malléables. Autrement dit, dès qu'on prend en compte le fait que différentes technologies sont possibles pour une même fonctionnalité, il est tout à fait possible qu'en fonction des prix, une technologie où deux brevets sont simultanément utilisés puisse être économiquement plus intéressante que deux autres technologies utilisant chacune l'un des deux brevets seulement, l'inverse pouvant par ailleurs se produire pour d'autres intervalles de variation des prix. ${ }^{13}$ Dès lors, les auteurs explorent une autre voie qui permet d'éviter un certain nombre de problèmes. Ils proposent que lors de la constitution d'un pool, on autorise chaque détenteur de brevet de pouvoir licencier de manière indépendante son propre brevet. La question des licences indépendantes ne prend en effet son sens que dans des situations intermédiaires entre la substituabilité parfaite et la complémentarité parfaite. Autrement dit, autoriser des licences indépendantes n'a de sens que pour des licences imparfaitement complémentaires ou imparfaitement substituables. Le point important à souligner ici est qu'en permettant des licences indépendantes, on crée en quelque sorte une concurrence potentielle aux offres du pool. Ce sont les effets de cette concurrence potentielle qu'explorent les auteurs, à la fois sur le plan théorique (Josh Lerner et Jean Tirole, 2004, 2014) et sur le plan empirique (Josh Lerner, Parag Pathak et Jean Tirole, 2006, Josh Lerner, Marcin Strojwas et Jean Tirole, 2007). Ils montrent ainsi que l'introduction du principe de licences indépendantes a deux effets positifs. Le premier est un effet d'incitation à former un pool conduisant

\footnotetext{
${ }^{13}$ De manière plus générale, Patrick Rey et Jean Tirole (2013) examinent une classe de fonction de demande emboitée, dans laquelle les choix des utilisateurs technologiques sont déterminés en deux temps. Dans un premier temps, un utilisateur choisit dans chaque classe technologique quels inputs acheter dans la classe, privilégiant ainsi la dimension de substituabilité des inputs au sein d'une même classe technologique. Dans un deuxième temps, un utilisateur décide d'adopter ou non la technologie, privilégiant ainsi la dimension de complémentarité.
} 
à la réduction du prix de la licence globale. Le second, dans une optique plus Schumpétérienne, est un effet d'incitation à l'innovation. Une extension de l'article de Josh Lerner et Jean Tirole (2004) à un cadre dynamique, proposée dans Patrick Rey et Jean Tirole (2013) montre néanmoins que l'existence de licences indépendantes n'est pas une condition suffisante pour assurer qu'un patent pool est proconcurrentiel. Une autre propriété doit lui être adjointe, imposant que la somme des prix des licences indépendantes ne doit pas dépasser le prix joint proposé par le patent pool pour cet ensemble de licences. L'approche adoptée pour savoir si un patent pool est pro ou anticoncurrentiel nous offre, là encore, une illustration de l'ingéniosité dont font preuve Jean Tirole et ses coauteurs pour proposer des solutions simples à des questions qui se posent avec insistance dans l'agenda des politiques des pouvoirs publics. L'Union Européenne semble d'ailleurs être la plus avancée dans l'adoption de ces solutions.

\section{b/ Standards technologiques}

Les standards technologiques ont une double fonction dans l'économie. D'une part, ils permettent aux consommateurs de bénéficier de l'usage de multiples biens et services, en s'affranchissant totalement des contraintes de compatibilité, du fait que ces biens et services sont offerts sur une même plateforme, et de ce fait rendus parfaitement compatibles. II suffit, pour s'en convaincre, de constater l'étendue des applications disponibles sur un smartphone, que celui-ci fonctionne sous la technologie IOS ou Android. D'autre part, les standards permettent aux producteurs de développer leurs produits en leur garantissant que la norme technologique proposée par le standard est relativement pérenne. Cette garantie offre une forte incitation à l'innovation. Mais au-delà de ces deux très appréciables avantages, l'actualité contemporaine révèle que les standards sont également un terrain d'affrontements juridiques récurrents et coûteux, sans que les coûts exorbitants de ces litiges ne soient favorables au moindre progrès technique ou économique. L'origine de ces conflits réside dans le fait que, lors de la constitution d'un standard technologique par une organisation professionnelle spécialisée (Standard Setting Organization, acronyme SSO), un certain nombre de brevets acquièrent un statut spécial du simple fait qu'ils font partie du standard choisi par l'organisation. Ils deviennent en effet des "Brevets Essentiels du Standard" (Standard Essential Patents, acronyme SEP). Le nombre de ces brevets, parfois exorbitant, nécessite un certain nombre de garde-fous pour éviter des dérives. Premièrement, il faut éviter qu'un détenteur de brevet essentiel n'exploite ex post son pouvoir de marché du simple fait que son brevet fait partie du standard adopté. Le même brevet n'aurait peut être pas bénéficié de ce pouvoir de marché si le standard adopté avait été différent. Deuxièmement, en décidant au sein d'un ensemble de technologies possibles laquelle est élue en tant que standard, l'organisme professionnel offre aux détenteurs de certains brevets, devenus du coup essentiels pour ce standard, une opportunité de développement considérable. On aurait pu imaginer qu'en contrepartie, l'organisme de standardisation exige ex ante, c'est-à-dire avant le choix même du standard, que les titulaires de brevets s'engagent sur un certain nombre de règles, dont notamment le prix des licences. Mais, jusqu'à présent, aucun SSO ne le fait. Par contre, un SSO est en droit de demander ex-post, c'est-à-dire après le choix définitif du standard, qu'un certain nombre de conditions soient satisfaites. Parmi elles, figurent l'engagement des détenteurs de ces brevets : i/ à ne pas entraver leur diffusion, ii/ à ne pas cacher un brevet pendant la constitution du standard pour recourir ensuite à des comportements opportunistes contre de présumés infracteurs; iii/ à proposer une licence collective ou des licences individuelles à des prix jugés équitables, raisonnables et non discriminatoires (ce que traduit l'acronyme anglais FRAND, pour Fair, Reasonable And Non Discriminatory). Mais, d'une part, le pouvoir de l'organisation professionnelle reste limité ex post, c'est-à-dire après le choix du standard. D'autre part, les critères recommandés de "prix juste et raisonnable" pour les licences ne sont pas d'une clarté éblouissante, sans compter qu'il revient à un tribunal et non au SSO de juger si ces critères sont satisfaits ou non. Les engagements ex post des détenteurs de ces brevets en tant que membres du SSO, n'ont empêché ni l'émergence de comportements opportunistes, ni les conflits juridiques sur l'interprétation qu'il convient de donner au qualificatif FRAND des prix de licence (David Encaoua et Thierry Madiès, 2014). Les procès récurrents faisant intervenir les six grands de l'industrie du numérique aux Etats-Unis (Microsoft, Apple, Facebook, Google, Amazon, Samsung) à propos des smartphones ou de différents logiciels propriétaires, en attestent amplement. De nombreux observateurs ont ainsi souligné que la notion de prix juste et raisonnable devait refléter le résultat d'une concurrence technologique ex ante et non pas d'une situation de monopole obtenue ex post après que la technologie standard ait été adoptée.

Partant de cette remarque, un travail récent de Josh Lerner et Jean Tirole (2013) développe un cadre alternatif original qui a pour objectif de limiter les défaillances associées aux standards technologiques. Ce travail permet de comprendre comment différentes inefficacités ou distorsions naissent de l'absence d'engagement en prix ex ante et montre quels types d'engagements permettraient de rétablir une véritable concurrence sans induire les mêmes distorsions. Sur le plan méthodologique, ce travail présente diverses originalités. L'une d'elles est que la notion de brevet essentiel est endogénéisée, plutôt que de considérer ce statut privilégié comme une donnée intrinsèque. Un brevet n'est pas intrinsèquement essentiel, indépendamment du choix d'un standard et donc indépendamment des engagements en prix initiaux. Ceci est très important, car aussi bien dans la pratique de fonctionnement des SSO que dans la littérature consacrée aux standards technologiques, la qualité de brevet essentiel semble avoir une logique intrinsèque. Remettre en question cette logique est particulièrement pertinent dans toutes les situations où il existe plusieurs voies de recherche différentes conduisant à des ensembles différents de brevets qui seront dits après coup essentiels selon le standard technologique adopté. Une autre originalité du travail de Josh Lerner et Jean Tirole (2013), de nature méthodologique, consiste à supposer que la demande des utilisateurs (qu'on peut également appeler des développeurs) s'exprime non plus en termes de brevets mais en termes de fonctionnalités, sachant qu'une même fonctionnalité peut être satisfaite par divers brevets en concurrence. Enfin, une troisième originalité, mais c'est loin d'être la dernière de cette remarquable contribution, est que le choix par un développeur des fonctionnalités qui correspondent à sa combinaison idéale $n^{\prime}$ est pas indépendant du choix des autres développeurs, ce que traduit le rôle important des externalités de réseaux.

L'article de Josh Lerner et Jean Tirole (2013) ne livre pas seulement une analyse théorique détaillée des inefficacités du système actuel de fonctionnement des SSO ; il propose une procédure à plusieurs étapes permettant de remédier à ces inefficacités.

Dans une première étape exploratoire, comme cela est déjà le cas, les membres du SSO examineraient la viabilité technique des différentes solutions technologiques, candidates à constituer un standard, sans se préoccuper des prix auxquels les licences seront mises sur le marché. Dans une seconde étape, toujours avant qu'un standard spécifique ne soit adopté, chaque membre du SSO s'engagerait sur un prix plafond auquel il accepterait de licencier son brevet, indépendamment du fait que celui-ci fera partie ou non d'un standard. Cet engagement préalable sur le prix maximum d'une licence est porté à la connaissance de chacun et ne doit donner lieu à aucune renégociation après le choix final du standard. Cette seconde étape est bien entendu originale par rapport aux pratiques en vigueur. Dans une troisième étape, les membres du SSO choisiraient alors un standard, sans se préoccuper des prix, comme cela se fait déjà. Dans une quatrième étape enfin, après que le standard ait été choisi, les détenteurs de brevets intervenant dans le standard pourraient regrouper leurs brevets dans un pool, et décider d'un prix de licence collective, comme c'est souvent le cas dans la pratique actuelle.

Les auteurs parviennent à montrer que l'inclusion de la seconde étape dans le processus a un effet très important. L'idée est à la fois simple et lumineuse. Deux cas sont à envisager. Dans le premier cas, les brevets sont substitutables entre eux avant l'adoption d'un standard spécifique. Dans ce cas, le prix plafond auquel s'engage chaque détenteur de brevet ex ante est nécessairement plus faible que le 
prix auquel il consent de licencier son brevet, lorsque celui-ci fait déjà partie du standard. De plus, chaque détenteur a d'autant plus intérêt à s'engager sur un prix faible ex ante que cela peut jouer en faveur d'un standard qui inclut son brevet. Dans le second cas où les brevets sont complémentaires, chaque détenteur a intérêt à fixer le prix le plus bas possible. Les auteurs montrent alors que ce processus de décision à quatre étapes conduit à une situation où le prix des licences est le même que celui qui prévaudrait en l'absence d'externalités de réseaux ou, dit autrement, le prix que paierait un utilisateur du brevet indépendamment de l'existence d'un standard. L'introduction d'un engagement sur le prix plafond avant le choix du standard conduit in fine, d'une part, à réduire le prix que devra payer le licencié d'une technologie apparaissant dans le standard et, d'autre part, à rapprocher la redevance payée pour une licence de la valeur véritable du brevet. On retrouve de la sorte un équilibre entre l'incitation à l'innovation et l'incitation à sa diffusion.

Les détenteurs de brevets, qui sont membres des SSO, accepteront-ils d'appliquer le processus suggéré ? La réponse semble être négative pour des raisons évidentes. Dès lors, ne convient-il pas d'introduire une certaine forme de régulation des standards par les pouvoirs publics, s'interrogent les auteurs? Par exemple, en tant qu'utilisateurs des technologies standardisées, les pouvoirs publics pourraient exiger l'introduction de la nouvelle procédure dans le processus de standardisation dans tous les cas où l'Etat est un acheteur important. Par ailleurs, les agences chargées du droit de la concurrence, étant elles-mêmes concernées par les distorsions induites par des licences de prix élevé, pourraient également favoriser la nouvelle procédure. Là encore on peut voir l'influence d'une pensée rigoureuse, soucieuse de l'intérêt public, et cherchant les modalités d'intervention opportunes des pouvoirs publics. En tout état de cause, les discussions sur l'adoption de cette procédure semblent être plus avancées dans l'Union Européenne qu'aux Etats-Unis.

\section{Théorie de la régulation: une refondation par Laffont et Tirole}

La régulation concerne deux types d'activités économiques : celles qui fonctionnent selon une logique de réseau où l'interconnexion entre différents opérateurs est cruciale et celles qui, étant régies par des rendements croissants, ressortent de la catégorie du monopole naturel. Cependant, comme les raisons d'une intervention publique pour réguler ces deux types d'activités et les modalités de cette intervention ne font pas appel aux mêmes principes, la distinction paraît justifiée. Nous présentons ci-dessous quelques uns des travaux de Jean Tirole sur la régulation de ces deux types d'activités.

\section{1 Doit-on réguler les activités de réseaux ? L'exemple des télécommunications.}

Peut-on se passer d'une régulation économique dans les activités de réseaux ? En d'autres termes peut-on remplacer une régulation économique spécifique de ces industries par une simple application des principes généraux du droit de la concurrence, tels que l'interdiction des pratiques de cartellisation et les abus de position dominante ? C'est à cette question, inspirée par l'actualité dans certains pays (déréglementation des télécommunications en Nouvelle Zélande par exemple) et motivée par l'absence d'une analyse théorique générale de la nature des défaillances du marché dans les activités de réseaux, que sont consacrés deux articles pionniers de Jean Jacques Laffont, Patrick Rey et Jean Tirole (1998 a et b, LRT dans la suite). Dans une économie de réseaux, deux sources de défaillance des marchés peuvent a priori être identifiées.

La première est que le plus souvent les opérateurs interconnectés sont peu nombreux. Ils peuvent donc utiliser les prix d'interconnexion comme des instruments pour s'entendre plutôt que pour s'affronter, remettant en cause le principe fondamental de la concurrence.

La seconde défaillance vient du fait que le comportement stratégique de l'opérateur dominant, dont la nature est héritée de l'histoire, peut handicaper sérieusement l'entrée d'un nouvel opérateur, en lui refusant par exemple l'accès à son réseau, utilisant pour cela un principe de forclusion comme on l'a vu plus haut.

Pour donner corps à ces deux possibilités, LRT examinent le cas d'une concurrence entre deux opérateurs téléphoniques asymétriques, dotés chacun d'un réseau spécifique et offrant des fonctionnalités différenciées horizontalement. Les clients potentiels ont le choix de l'opérateur auquel ils s'abonnent et leur surplus net décroit avec les prix fixés par les deux opérateurs et croit avec le nombre total d'abonnés raccordés, du fait d'évidentes externalités de réseau. Plus l'écart entre la fonctionnalité préférée d'un utilisateur et celle offerte par l'opérateur est grande, plus forte est la désutilité subie, comme dans tout modèle de différenciation horizontale. Le paramètre de désutilité sert notamment à évaluer l'intensité de la concurrence entre les opérateurs. Plus ce paramètre est faible, plus la substituabilité entre les deux réseaux est élevée et plus la concurrence est intense.

LRT distinguent les appels "intra" et "extra". Un appel "intra" (désigné communément en télécommunications comme un appel "on net") connecte deux utilisateurs raccordés au même réseau. Dans ce cas l'appel sortant et l'appel entrant transitent par le même réseau physique. Un appel "extra" (désigné comme un appel "off net") connecte des utilisateurs raccordés à deux réseaux différents. Un coût $d$ 'accès (acheminement, connexion, etc.) à l'autre réseau est alors encouru. Deux hypothèses de base concernant ces deux types d'appels prévalent. La première stipule que la proportion des appels "on net" d'un réseau est égale à la proportion des consommateurs raccordés à ce réseau. La seconde est une hypothèse de symétrie : la charge d'accès payée par l'opérateur d'un réseau pour faire accéder un appel d'un de ses abonnés vers un abonné de l'autre réseau est la même sur les deux réseaux. Deux cas sont alors examinés selon la structure des prix d'accès à un réseau. Dans le premier cas (LRT, 1998 a), l'abonné d'un réseau donné paye le même prix que son appel se termine sur ce réseau ou sur l'autre réseau. Ce cas correspond à une absence de discrimination selon la destination de l'appel. Dans le deuxième cas (LRT, 1998 b), un opérateur peut discriminer, en pratiquant notamment une tarification plus faible pour des appels à destination de son réseau (par exemple formule des "on-net" illimités, choix de correspondants à tarifs plus faibles, amis, famille, etc.), ce qui favorise évidemment le raccordement à un même réseau. Lorsque les prix des appels diffèrent selon le réseau de terminaison, les externalités de réseaux deviennent un facteur crucial de choix des consommateurs.

Les auteurs parviennent à plusieurs résultats auxquels conduirait la concurrence en l'absence de régulation. Premièrement, l'existence d'un équilibre de marché n'est pas assurée lorsque la charge d'accès et/ou l'intensité de la concurrence sont trop élevés. Deuxièmement, en l'absence de régulation, les opérateurs de réseaux ont intérêt à appliquer des prix différents pour les appels "on-net" et "off-net", c'està-dire à pratiquer des prix discriminants. Même lorsqu'un équilibre de marché existe, les différences de prix peuvent conduire à des situations où l'un des deux opérateurs est éliminé. Une des raisons fondamentales est qu'en l'absence de régulation, la discrimination en prix selon les appels "on-net" et "off-net" n'est pas justifiée par les différences en termes de coûts ou d'élasticités des fonctions de demande, et de ce fait, conduit à une mauvaise allocation des demandes. Troisièmement, la discrimination par les prix peut être conçue comme une barrière à l'entrée. Quatrièmement enfin, la discrimination des prix n'a pas que des effets négatifs. Elle peut accroître la concurrence entre les opérateurs conduisant à des prix d'usage plus faibles, en dépit du fait que les coûts de chaque opérateur sont plus élevés du fait des charges d'accès off-net que fait subir l'opérateur concurrent.

Tous ces problèmes liés à une absence de régulation donnent lieu évidemment à un examen approfondi lorsque l'activité est régulée, comme le sont par exemple les télécommunications. En France, sous l'autorité de l'ART d'abord puis de l'ARCEP, le taux de pénétration du 
mobile a progressé, le nombre d'opérateurs s'est accru, le prix des communications a lui-même baissé. Mais, c'est surtout l'arrivée du quatrième opérateur, Free, qui a eu le plus d'impact. Toutes les modalités de la régulation des télécommunications ne sont pas encore stabilisées, notamment le prix des terminaisons d'appel, et les comportements de certains opérateurs ont été parfois contestés, non pas auprès de I'ARCEP, mais auprès de l'Autorité de la Concurrence. Une décision récente de l'Autorité de Concurrence (12-D-24 du 13 décembre 2012) portant sur la téléphonie mobile souligne un certain nombre de failles dans le secteur de la téléphonie mobile. "Alors que le marché est depuis quelques années déjà un marché de renouvellement (changement d'opérateur et/ou de terminal) et non plus un marché de primo-accédants, la part des clients sous engagement devrait tendre naturellement à se réduire. Or...ce chiffre est en augmentation constante depuis le troisième trimestre 2005, date à laquelle l'ARCEP à commencé à suivre cet indicateur". Cependant, les opérateurs qui désirent montrer que la concurrence est très vive dans le secteur de la téléphonie mobile utilisent la notion de taux de "churn" qui mesure la part des clients résiliant leur abonnement au cours d'une période donnée (un mois par exemple). lls comparent pour cela les valeurs de ce taux dans deux activités : l'internet et la téléphone mobile. Alors que pour l'internet, la part des clients résiliant leur abonnement à un opérateur est inférieur à 1\%, elle est de 2 à $3 \%$ dans le mobile. Mais comme l'observe l'Autorité de la Concurrence dans la décision précitée, "le taux de "churn " mobile est un indicateur non déterminant, car une part importante des individus qui résilient leur ligne se réabonne dans la foulée chez le même opérateur". Tous ces comportements illustrent bien que la régulation d'une activité complexe comme la téléphonie mobile est à la fois nécessaire mais est loin d'être parfaitement stabilisée.

\section{IV.2 L'héritage en matière de théories de la régulation des monopoles naturels}

Les activités de services publics se décomposent en général en deux types : celles où des rendements croissants prévalent, qui sont dites des structures de monopole naturel (activités de type1) et celles où la concurrence peut s'exercer pour peu que les barrières stratégiques à l'entrée soient levées (activités de type 2). La première partie de cet article ayant déjà traité les questions relatives aux barrières à l'entrée dans les activités de type 2, il nous faut examiner les contributions de Jean Tirole à la régulation des activités de type 1.

La question de savoir comment l'Etat peut intervenir dans la régulation d'un monopole naturel a toujours été omniprésente dans la littérature économique. Avant même que cette question ne soit envisagée sous l'angle de l'analyse économique, des approches pragmatiques de cette question ont eu un rôle important notamment en France, sous la férule de brillants ingénieurs économistes. Leur mission dans les ministères techniques était multiple : octroyer des concessions, en surveiller l'exploitation, fixer les tarifs, etc. Une brève illustration concernant les questions de tarification des voyages en train nous permet de saisir l'ingéniosité des solutions proposées. Comment concevoir des tarifs couvrant les coûts très élevés des infrastructures ferroviaires (en situation de monopole naturel donc) tout en permettant au plus grand nombre de prendre le train en ne payant qu'un prix très faible ? La solution proposée par l'ingénieur économiste français Jules Dupuit (1854) consiste à imaginer un mécanisme faisant révéler aux voyageurs potentiels leur disponibilité à payer une place en train. Le mécanisme, équivalent à une discrimination du $2^{\text {ème }}$ degré, consiste à proposer trois classes de tarifs selon des degrés de confort très contrastés, afin que les voyageurs s'auto-sélectionnent: ceux ayant les plus faibles revenus acceptant de voyager dans des conditions de confort spartiates (troisième classe) mais à des prix très bas, ceux aux revenus les plus élevés acceptant de payer des prix très élevés pour bénéficier des très bonnes conditions de confort (première classe), et ceux aux revenus intermédiaires choisissant le prix et le confort intermédiaires (deuxième classe). En un mot, avant même que le vocable précis de régulation n’ait été introduit, le rôle des ingénieurs économistes était de faire de la régulation sans le savoir, comme Monsieur Jourdain faisait de la prose. De plus, comme le montre cet exemple, le rôle de l'information incomplète (les disponibilités à payer des voyageurs) était déjà présent, et l'appel à un mécanisme révélateur assurant la fonction de faire voyager le plus grand nombre d'agents en leur faisant révéler (indirectement) leur disponibilité à payer était déjà là.

Les approches économiques de la tarification d'un monopole naturel ont commencé à apparaître au $20^{\text {ème }}$ siècle. Un travail célèbre sur la fiscalité par Frank Ramsey (1927) ouvrait la voie à une approche spécifique du problème de la tarification des prix d'un monopole naturel par Marcel Boiteux (1956). Cet auteur, autre exemple de contribution significative d'un ingénieur économiste français, proposait une tarification basée sur la maximisation du surplus des consommateurs sous la contrainte d'équilibre budgétaire du monopole. Cette maximisation conduit à un niveau de prix s'écartant d'autant plus du coût marginal que l'élasticité de la demande est faible. La tarification qui s'en suit, dite à la "Ramsey-Boiteux", correspond bien à un optimum de second rang, dont on sait que l'implémentation se heurte à une énorme difficulté : ni la fonction de demande ni la fonction de coût ne sont connues du régulateur. La question s'est donc posée de savoir s'il ne valait pas mieux financer l'activité d'un monopole naturel par des fonds publics, plutôt que d'imposer des prix fixés par la puissance publique. Dans un article célèbre, Ronald Coase (1946) revenait sur cette question en concluant à l'impossibilité d'implémenter l'optimum de premier rang, du fait des problèmes informationnels que soulève la mesure du surplus social, défini par la différence entre la disponibilité à payer des consommateurs et le coût de production de l'entreprise. Les travaux suivants, surgis dès la fin des années 1970, ont abandonné l'idée d'une fixation des prix d'un monopole naturel par les pouvoirs publics. Ils mettaient plutôt l'accent sur une régulation par incitation, consistant à déléguer les décisions de prix et de coûts à l'entreprise régulée, en laissant celle-ci s'approprier l'accroissement de profit engendré par une éventuelle réduction des coûts. Un premier travail par Ingo Vogelsang et Jörg Finsinger (1979) ouvrait la voie. Le mécanisme dynamique proposé par ces auteurs est défini par une procédure itérative dans laquelle les variables de prix, de quantités et de coûts afférents à une période donnée déterminent les prix que le régulateur autorise à employer pour la période suivante. Plus précisément, les prix autorisés pour une période sont tels que la recette de la période, calculée en fonction des prix autorisés, n'excède pas le coût de la période précédente. Ce mécanisme apparemment très simple a une remarquable propriété : il converge à long terme vers les prix à la Ramsey - Boiteux, permettant ainsi d'implémenter l'optimum de second rang. Martin Loeb et Wesley Magat (1979) vont un peu plus loin en proposant un mécanisme permettant d'implémenter l'optimum de premier rang dans une situation où le régulateur connaît la fonction de demande mais pas la fonction de coût. Dans ce mécanisme, le monopole propose un prix et le régulateur subventionne l'entreprise régulée d’un montant égal au surplus du consommateur évalué au prix proposé par le monopole. Le modèle de Loeb et Magat souffre d'une critique fondamentale. En accordant à l'entreprise régulée l'intégralité du surplus social, le modèle ignore les aspects distributifs du problème, consistant en la prise en compte des intérêts aussi bien des consommateurs que de l'entreprise régulée. ${ }^{14}$ Le modèle suivant de David Baron et Roger Myerson (1982) se rapproche le plus d'une véritable théorie de la régulation incitative, dans la mesure où ce modèle endogénéise véritablement le choix du mécanisme, en s'appuyant sur le principe de révélation. De plus, le modèle intègre une fonction de surplus global pondéré où la rente de l'entreprise régulée a une pondération plus faible que celle attribuée au surplus des consommateurs. Cependant, le modèle n'intègre pas le coût social des fonds publics. De plus, dans ce modèle, seule la fonction de coût est supposée non connue du régulateur, la fonction de demande étant supposée connaissance

\footnotetext{
${ }^{14}$ Finalement, ce modèle exploite une propriété bien connue des modèles d'agence, à savoir que si un agent neutre par rapport au risque est désigné comme le créancier résiduel du surplus, l'efficacité allocative et l'efficacité technique peuvent être atteints.
} 
commune de l'entreprise régulée et du régulateur. Par ailleurs, le modèle ne prend en compte que la variable d'antisélection. Les auteurs parviennent ainsi à construire le transfert socialement optimal, sous les contraintes respectives de participation et d'incitation, chacun des types d'entreprise devant être incité à choisir l'option qui lui correspond. Le résultat le plus notable, qu'on retrouvera d'ailleurs dans les mécanismes d'incitation ultérieurs, est que pour faire révéler son véritable type à l'entreprise la plus efficace (celle dont le coût marginal est le plus faible), le régulateur doit lui laisser une rente positive, rente qui n'existerait pas si l'information était parfaite et qu'on qualifie pour cette raison de rente informationnelle. Le montant de cette rente est une fonction croissante de l'écart entre les coûts possibles de la firme régulée. Une étape supplémentaire a ensuite été franchie par David Sappington (1982). En endogénéisant la fonction de coût par la prise en compte d'actions spécifiques menées par l'entreprise régulée pour réduire son coût (aléa moral), David Sappington élargissait le problème. Mais en restreignant l'analyse à des contrats linéaires, le problème de savoir quel devait être le contrat optimal restait non résolu.

Une généralisation, opérée sur au moins trois points, a été apportée par Jean Jacques Laffont et Jean Tirole d'abord dans leur article de 1986, puis dans l'ouvrage de 1993. Le premier point a consisté en la prise en compte simultanée des deux variables informationnelles correspondant à l'antisélection et à l'aléa moral, sans aucune restriction a priori sur le mécanisme. Le second point a consisté en l'introduction du coût social que représente la rente informationnelle, du fait d'un financement par la fiscalité. Le troisième point a consisté à prolonger le modèle de base de la régulation d'un monopole naturel dans de multiples directions, en introduisant à la fois des aspects dynamiques (contrats de long terme) et des aspects institutionnels (capture de l'autorité de régulation). La prise en compte de ces différents éléments caractérise le renouveau de la théorie de la régulation économique et de sa mise en œuvre, cette dernière étant à l'origine d'importantes réformes en matière d'intervention publique dans les activités en monopole naturel. ${ }^{15}$ Le grand mérite de l'ouvrage monumental que Jean Jacques Laffont et Jean Tirole (1993) ont consacré à ce thème, et qui reprenait une série de travaux menés en collaboration pendant une dizaine d'années, est finalement triple. Premièrement, l'approche adoptée prend simultanément en compte les trois problèmes auxquels est confrontée la puissance publique pour réguler un monopole naturel: i/ comment inciter le monopole à se rapprocher au mieux de l'efficacité allocative qui impose une tarification au coût marginal; ii/ comment inciter le monopole à se rapprocher au mieux de l'efficacité productive qui lui impose de minimiser le coût de production; iii/ comment forcer le monopole à se rapprocher au mieux de l'efficacité distributive qui impose de rendre aussi faible que possible un financement public, nécessairement distorsif. Deuxièmement, la solution proposée par Jean Jacques Laffont et Jean Tirole répond à un besoin largement éprouvé durant une période où les performances des entreprises régulées dans les services publics n'étaient guère encourageantes, qu'il s'agisse des "private utilities" aux Etats Unis ou des opérateurs publics en Europe, dont ni la gouvernance ni les objectifs ne paraissaient clairement explicités. (David Encaoua, 1991). Troisièmement, l'opposition que I'on fait parfois entre régulation publique et libéralisation par l'ouverture à la concurrence s'avère totalement erronée. Dans I'Union Européenne comme aux Etats Unis, le nouvel objectif public a été d'améliorer la structure des incitations des mécanismes de régulation et non d'outrepasser la nécessité de l'intervention publique. Les transformations qui ont eu lieu dans différents services publics de l'économie comme les télécommunications, le transport aérien, le transport ferroviaire, l'électricité, le gaz, etc. se caractérisent par une combinaison complexe de principes gouvernant la réforme réglementaire elle-même et de mesures publiques pour favoriser la concurrence et l'entrée de nouveaux opérateurs. Le rôle précurseur de différents auteurs (Roger Guesnerie et Jean Jacques Laffont, 1984, Bernard Caillaud, Roger Guesnerie, Patrick Rey et Jean Tirole, 1988) dans l'analyse économique de ces transformations, a été des plus importants.

\section{IV.3 Mécanismes de régulation les plus fréquents : une typologie}

Les mécanismes de régulation les plus fréquemment utilisés se caractérisent selon deux critères. Le premier critère distingue les mécanismes selon qu'ils incluent ou non des transferts financiers de la part de la puissance publique à l'entreprise régulée. Le second critère porte sur la puissance du mécanisme, mesurant son caractère plus ou moins incitatif en termes de réduction des coûts. Nous esquissons ici une description des mécanismes les plus usuels en suivant la présentation de Philippe Gagnepain (2001)

1. La régulation selon le coût du service : le régime du "cost plus"

Partons d'une situation mettant en présence une autorité publique et une entreprise fournisseur d'un bien ou d'un service. L'autorité publique peut être l'Etat, dans sa fonction d'acheteur, comme par exemple dans le cas d'une commande du Ministère de la Défense, ou une administration locale, comme dans le cas du transport urbain par exemple. Dans les deux cas, un cahier des charges est rédigé par la puissance publique et moyennant sa satisfaction, un paiement est assuré à l'entreprise prestataire, pour lui rembourser l'intégralité des coûts qu'elle a supporté. C'est le régime dit du "cost plus" qui est évidemment un mécanisme de puissance nulle, dans la mesure où il n'incite nullement l'entreprise à réduire ses coûts puisque le paiement est indépendant de la performance réalisée. Le prestataire n'est le créancier résiduel d'aucun profit.

2. La régulation selon un transfert indépendant du coût effectif : le régime du "prix fixe"

La situation polaire du cas précédent est celle où la puissance publique paye au prestataire un montant forfaitaire totalement indépendant des coûts subis. Ce forfait est supposé assurer l'équilibre financier du prestataire, mais en tout état de cause, c'est le prestataire qui supporte les dépassements éventuels de coûts de la même façon qu'il s'en approprie le bénéfice si les coûts s'avèrent plus faibles que ceux de l'équilibre financier anticipé par la puissance publique. C'est le régime dit du "prix fixe" qui est évidemment un mécanisme de puissance maximale puisqu'il incite le prestataire à réduire au maximum ses coûts, le prestataire devenant en effet le créancier résiduel de la rente qu'il aura contribué à créer par l'effort employé à réduire les coûts.

3. La régulation selon des transferts dépendant des coûts : le régime des mécanismes incitatifs

Les contrats de régulation intermédiaires entre les deux cas polaires précédents, sont ceux où la puissance publique et le prestataire de services partagent les risques liés à l'écart entre les coûts effectifs et les coûts anticipés, ceux-ci étant censés assurer l'équilibre financier du prestataire. Supposons que les recettes du prestataire proviennent de la commercialisation de sa production et considérons un cadre comptable où les recettes commerciales sont appropriées par l'entreprise et les coûts supportés par elle. Supposons en outre que la puissance publique propose un transfert financier au prestataire, qu'on peut représenter ex ante par la fonction affine $t\left(C, C^{a}\right)=a+(1-b)\left(C-C^{a}\right)$ où $C^{a}$ correspond au niveau des coûts anticipés ex ante et censés assurer l'équilibre financier du prestataire, et $C$ est le montant des coûts effectifs observés ex post, c'est-à-dire après que l'offre du prestataire ait été réalisée. Le paramètre a $\geqslant 0$ correspond à la partie forfaitaire du transfert et le paramètre b mesure la fraction du dépassement des coûts d'exploitation par rapport à l'équilibre financier anticipé, que supporte l'entreprise régulée, avec $b \in[0,1]$. La fraction complémentaire de ce dépassement de coût, représentée par la valeur de $(1-b)$, est financée par un transfert du régulateur vers l’entreprise régulée.

\footnotetext{
${ }^{15}$ Voir Bernard Caillaud et al. (1988).
} 
On retrouve alors comme cas particuliers les deux mécanismes polaires précédents. Le cas $b=0$ correspond au régime du "cost plus" dont on a dit qu'il était de puissance nulle. Le cas $b=1$ correspond au régime du "prix fixe" dont on a dit qu'il était de puissance maximale. Les cas intermédiaires, caractérisés par des valeurs du paramètre $b$ telles que $0<b<1$, correspondent à des régimes de régulation incitative dont la puissance est précisément mesurée par la valeur de $b$. La détermination d'une valeur de ce paramètre reflète un compromis entre deux objectifs opposés, d'une part celui d'inciter à la réduction du coût et d'autre part, celui de réduire la rente laissée à l'entreprise régulée. La recherche de ce compromis constitue tout l'enjeu de la détermination d'un mécanisme incitatif socialement optimal et c'est précisément à ce problème que Jean Jacques Laffont et Jean Tirole (1986) ont apporté une contribution décisive.

Les schémas types dans les situations où un transfert financier n'est pas envisageable sont présentés ci-dessous.

4. La régulation par le taux de rendement ("rate of return")

La régulation par le taux de rendement a longtemps été pratiquée, notamment aux Etats Unis. Elle consiste à imposer à l'entreprise régulée un prix de vente de son bien dont la détermination procède en deux temps. Dans un premier temps, le régulateur évalue le stock de capital de l'entreprise régulée à partir des investissements antérieurs en tenant compte de la dépréciation du capital et des besoins futurs en capital durant une période donnée. Un taux de rendement égal ou légèrement supérieur au coût de marché du capital est fixé. Le niveau des recettes autorisées est alors la somme des coûts d'exploitation et du rendement du capital au taux fixé. Dans un deuxième temps le prix autorisé est déterminé de manière à ce que les recettes effectives obtenues en multipliant ce prix par la quantité produite de l'entreprise régulée, soient égales au niveau des recettes autorisées. Ce type de régulation est soumis à plusieurs critiques. Premièrement, comme sous le principe du "cost plus", le mécanisme de la régulation par le taux de rendement ne conduit à aucune incitation pour que le coût soit le fruit d'une bonne gestion des ressources. Deuxièmement, Harvey Averch et Leland Johnson (1962) ont montré que la régulation par le taux de rendement conduisait à une inefficacité productive dans le choix des combinaisons de facteurs. Dès lors que la valeur du taux de rentabilité du capital autorisé par la puissance publique est légèrement supérieure au taux du marché, l'entreprise régulée privilégie le facteur capital au détriment du facteur travail. A l'inverse, si la valeur autorisée du taux de rentabilité du capital est inférieure ou égale au taux du marché, l'entreprise régulée, de statut privé aux Etats Unis, peut être dissuadée de procéder à des investissements en capital. Malgré ces réserves, la régulation d'un certain nombre d'opérateurs privés offrant des services publics ("private utilities") aux Etats Unis, comme l'opérateur téléphonique ATT, a longtemps été de ce type.

5. La régulation par un prix plafond ("price cap")

Le principe de la régulation par un prix plafond consiste à ce que l'autorité publique fixe un prix de vente maximal pour un panier pondéré de biens, quitte ensuite à ce que l'entreprise régulée choisisse ses prix individuels de façon que leur pondération ne dépasse pas le prix plafond. Ce type de régulation s'est beaucoup développé en Grande Bretagne durant les années qui ont suivi la privatisation des entreprises de service public dont les recettes provenaient directement de la vente au public. La régulation par un prix plafond possède de bonnes propriétés incitatives, comme la régulation de type prix-fixe examinée plus haut. Si les coûts sont trop élevés, une régulation par le prix-plafond ne permet pas des recettes commerciales suffisantes pour couvrir entièrement les coûts. De ce fait, ce type de régulation incite l'entreprise régulée à réduire ses coûts. Toutefois, la question principale concerne la détermination du prix plafond. Un prix trop élevé conduirait à laisser des rentes importantes aux entreprises. Inversement un prix trop faible pourrait conduire à une dégradation de la qualité des services offerts, comme cela a parfois été observé. Pour éviter ces risques, une révision périodique des prix plafonds (généralement tous les trois à cinq ans) a lieu en fonction de l'évolution des coûts des facteurs, des conditions économiques générales et des perspectives sur les gains de productivité. Au Royaume Uni, le taux de croissance autorisé du plafond a été déterminé par la formule RPI-X où RPI (Resale Price Index) désigne le taux de croissance de l'indice des prix à la consommation, et $\mathrm{X}$ désigne le taux anticipé de croissance du progrès technique. ${ }^{16}$

6. Une typologie

Tous les mécanismes de régulation usuels que nous venons de décrire donnent lieu à une typologie (tableau 1) construite à partir des deux critères mentionnés plus haut : existence ou non de transferts financiers et puissance du mécanisme. On exprime le transfert éventuel par l'expression examinée plus haut : $t\left(C, C^{a}\right)=a+(1-b)\left(C-C^{a}\right)$ où le paramètre $b(0 \leqslant b \leqslant 1)$ mesure la proportion du dépassement supporté par l'entreprise du coût effectif ex post $C$ par rapport au coût anticipé ex ante $C^{a}$. La typologie ci-dessous (tableau 1) distingue deux cas selon que l'entreprise est passible ou non de recevoir des transferts. La régulation incitative est celle qui, prenant en compte les asymétries d'information de base entre le régulateur (agissant comme le principal dans une relation d'agence) et le régulé (l'agent), détermine un mécanisme socialement optimal qui, d'une part, incite l'agent à fournir les efforts nécessaires pour réduire le coût d'exploitation en lui donnant un rôle de "créancier résiduel" d'une partie des gains ainsi obtenus, et, d'autre part, réduit le coût social du transfert que verse le régulateur au régulé. C'est fondamentalement l'idée de base exploitée dans le travail de Jean Jacques Laffont et Jean Tirole (1986) que nous examinons ci-dessous.

\begin{tabular}{|l|l|l|}
\hline Puissance du mécanisme & Transferts autorisés & Transferts non autorisés \\
\hline Faible (b proche de 0) & $\begin{array}{l}\text { Régulation de type coût du service } \\
\text { ("cost plus") }\end{array}$ & $\begin{array}{l}\text { Régulation du prix selon le taux de } \\
\text { rendement ("rate of return") }\end{array}$ \\
\hline Intermédiaire (b compris entre 0 et 1) & $\begin{array}{l}\text { Régulation incitative équilibrant la } \\
\text { réduction de coût et la réduction de } \\
\text { la rente informationnelle }\end{array}$ & $\begin{array}{l}\text { Régulation du prix par contrats } \\
\text { incitatifs }\end{array}$ \\
\hline Forte (b proche de 1) & Régulation de type "forfait fixe" & $\begin{array}{l}\text { Régulation selon un prix plafond } \\
\text { ("price cap") }\end{array}$ \\
\hline
\end{tabular}

Tableau 1 : Types de réglementation selon la puissance du mécanisme et selon que les transferts sont autorisés ou non

\section{IV.4 Le modèle de base de Laffont-Tirole}

Le renouveau opéré par le modèle de base de Jean Jacques Laffont et Jean Tirole (1986) et de ses prolongements présentés dans le monumental ouvrage publié par les mêmes auteurs quelques années après (1993), résulte de plusieurs points. Premièrement, il s'agissait de répondre à une question qui était loin d'être simple. Comment réguler l'unique entreprise prestataire, sachant que le régulateur n'a qu'une idée très imprécise sur le coût de fourniture du bien ou du service en question ? Deuxièmement, il s'agissait également de prendre

\footnotetext{
${ }^{16}$ Pour de plus amples détails, le lecteur peut consulter Mark Armstrong, Simon Cowan et John Vickers (1994) et David Sappington \& David Weisman (2010).
} 
en compte les éventuelles défaillances des processus de régulation sur lesquels George Stigler (1971) avait jeté un doute profond: loin de servir les intérêts des consommateurs, la régulation ne serait qu'une forme de protectionnisme dont bénéficient essentiellement les entreprises régulées. Troisièmement enfin, il s'agissait d'étudier les processus de régulation dans un cadre temporel dynamique et d'analyser le rôle de différents instruments (ouverture à la concurrence, enchères, etc.).

Pour développer brièvement ces points ${ }^{17}$, nous suivrons la présentation de Philippe Gagnepain (2001) et pour simplifier, nous nous placerons dans le cas où le monopole est mono-produit.

Pour produire la quantité $\mathrm{Q}$, le coût du monopole naturel dépend de trois facteurs : $\mathrm{i} /$ la quantité $\mathrm{Q}$ proprement dite ; ii/ une variable d'aléa moral, notée e, qui synthétise l'ensemble des actions que l'entreprise peut entreprendre pour réduire ses coûts, sachant que, d'une part, le niveau e est non observé par le régulateur et, d'autre part, la réalisation de ces actions entraîne un coût pour l'entreprise dont l'équivalent monétaire $\Psi(\mathrm{e})$ est croissant avec e à un taux croissant, la fonction $\Psi($. ) étant supposée connue du régulateur ; iii/ une variable d'antisélection, notée $\theta$, qui renvoie au type de l'entreprise (mesuré par exemple par sa productivité), non observé par le régulateur : plus $\theta$ est élevé, plus l'entreprise est efficace et plus son coût est faible. Le support de $\theta$ est connu [ $\theta_{-}, \theta^{+}$] ainsi que la distribution $\mathrm{F}$ et la densité $\mathrm{f}$ sur ce support. Enfin le coût du monopole naturel peut être affecté par des aléas communs à tout processus de production, qu'on représente en général par un bruit blanc. Le coût d'exploitation de l'entreprise régulée $s^{\prime}$ 'écrit $C(Q, e, \theta)$ où $C_{1}>0, C_{2}<0$ et $C_{3}<0$. Le coût d'exploitation ex post est observé par le régulateur mais sa ventilation selon les trois facteurs $(Q$, e, $\theta)$ ne l'est pas. L'hypothèse d'un financement distorsif d'un éventuel transfert financier de la puissance publique à l'entreprise régulée est introduite sous la forme du coût des fonds publics : 1 euro de transfert public coûte à la collectivité $(1+\lambda)$ euros où $\lambda>0$ est le coût social des fonds publics. Cela implique que le bien être du contribuable baisse de $(1+\lambda)$ euros pour chaque euro de recette fiscale que le gouvernement prélève pour financer le transfert. La fonction de demande est supposée connue du régulateur. Examinons la situation où les transferts de l'Etat vers l'entreprise régulée sont possibles. On note $R(Q)$ le montant des recettes commerciales engendrées par la vente de la quantité $Q$ par l'entreprise régulée et par $\mathrm{V}(\mathrm{Q})$ le surplus social brut engendré par cette quantité. Associés à un transfert brut $t$ de l'Etat vers l'entreprise régulée, la recette commerciale $R(Q)$ et le coût $C(Q, e, \theta)$ engendrent un revenu total de l'entreprise régulée, donné par : $Y=t+R(Q)-C(Q, e, \theta)$. Comme le coût de fourniture de l'effort e que supporte l'entreprise régulée est $\Psi(e)$, on en déduit que le revenu net de l'entreprise est donné par $U(Q, e, \theta, t)=Y-\Psi(e)=t+R(Q)-C(Q, e, \theta)-\Psi(e)$. En supposant que l'entreprise est neutre par rapport au risque, son profit peut être directement mesuré par son revenu net $U$.

Du surplus social brut $\mathrm{V}(\mathrm{Q})$ on soustrait le coût des fonds publics, induit par la distorsion qu'impose le financement du transfert par une taxe fiscale. On parvient ainsi à l'expression suivante du bien être social net dont il s'agit de maximiser l'espérance par rapport à $\theta$ : $W(Q, e, \theta, t)=V(Q)-Z(Q, e, \theta, t)$ où $Z(Q, e, \theta, t)=(1+\lambda)[C(Q, e, \theta)+\Psi(e)]+\lambda U(Q, e, \theta, t)$. Un mécanisme direct est défini par l'offre d'un transfert $\mathrm{t}(\theta)$ à un agent de type $\theta$. II s'agit donc de déterminer le mécanisme socialement optimal t $(\theta)$, c'est-à-dire celui qui maximise l'espérance du bien être social $\mathrm{W}(\mathrm{Q}, \mathrm{e}, \theta, \mathrm{t})$ par rapport à $\theta$ dont la densité $\mathrm{f}$ est supposée connue sur le support [ $\theta$, $\left.\theta^{+}\right]$, qui soit à la fois incitatif et réalisable. Ce mécanisme est obtenu en faisant appel au principe de révélation, selon lequel, s'il existe une stratégie optimale pour une firme de type $\theta$ à qui on propose le mécanisme $t$, alors on peut se restreindre au choix d'un mécanisme direct où l'entreprise annonce directement son type $\theta$. Elle peut soit mentir soit révéler son vrai type. Le mécanisme est dit révélateur s'il incite l'entreprise à révéler son vrai type. L'idée principale du "mechanism design" est donc de trouver un mécanisme direct révélateur. Notons $e^{*}(\mathrm{Q}, \mathrm{C}, \theta, \mathrm{t})$ l'effort optimal que choisirait l'agent de type $\theta$ confronté au mécanisme t et à la nécessité de produire $Q$ au coût $C$. On peut de la sorte se limiter à un problème ne comportant plus que la variable d'antisélection $\theta$. Le mécanisme consiste ainsi à demander à l'agent d'annoncer son type. Supposons que l'agent de type $\theta$, confronté au mécanisme, annonce une valeur $\theta^{\prime}$ différente de son vrai type. Le résultat décrit par l'ensemble $\left\{\left(\mathrm{Q}\left(\theta^{\prime}\right), \mathrm{C}\left(\theta^{\prime}\right), \mathrm{t}\left(\theta^{\prime}\right)\right\}\right.$ dépend alors de l'annonce $\theta^{\prime}$ faite par l'entreprise. Lorsqu'un agent de type $\theta$ annonce $\theta^{\prime}$, l'utilité de cet agent (et donc sa rente sous l'hypothèse de neutralité vis-à-vis du risque) s'écrit : $U\left(\theta^{\prime}, \theta\right)=t\left(\theta^{\prime}\right)-\Psi\left[e^{*}\left(Q\left(\theta^{\prime}\right), C\left(\theta^{\prime}\right), \theta\right)\right]$. Le mécanisme $\mathrm{t}$ (.) doit donc satisfaire deux contraintes.

La première est une contrainte d'incitation qui s'écrit : $U(\theta, \theta) \geqslant U\left(\theta^{\prime}, \theta\right) \forall\left(\theta^{\prime}, \theta\right) \in\left[\theta_{-}, \theta^{+}\right]^{2}$. Cette contrainte exprime que quel que soit le type de l'agent, annoncer son vrai type (c'est à dire sa productivité) est toujours préférable à mentir en surestimant ou en sous estimant son inefficacité. Pour que le mécanisme proposé incite l'agent à annoncer son vrai type, il est donc nécessaire que son annonce maximise son utilité lorsqu'il dit la vérité. La contrainte d'incitation conduit ainsi à une condition du premier ordre. Notons ici que le régulateur doit permettre à l'agent $\theta$ de s'approprier l'utilité $U(\theta, \theta)$ pour qu'il annonce son vrai type. Cette utilité correspond précisément à la rente informationnelle laissée par le mécanisme à l'agent $\theta$. Laffont et Tirole (1986) montre que la rente $U(\theta, \theta)$ laissée à l'agent $\theta$ est une fonction croissante de $\theta$, ce qui signifie que la rente laissée à un agent est d'autant plus élevée que l'agent est plus efficace.

La deuxième contrainte est une contrainte de participation qui s'écrit : $U(\theta, \theta) \geqslant 0 \forall \theta \in\left[\theta_{-}, \theta^{+}\right]$. Quel que soit son type, le profit d'un agent doit être non négatif pour qu'il accepte de participer au mécanisme.

Sans entrer ici dans le détail de la détermination du mécanisme socialement optimal, on peut répertorier quelques résultats significatifs. Seul l'agent le moins efficace $\theta=\theta$ obtient une rente nulle : $U(\theta, \theta)=0$. Notons $\varphi(\theta)=U(\theta, \theta)$ la rente laissée à l'agent $\theta$. En écrivant que $\varphi(\theta)$ est le maximum en $\theta^{\prime}$ de $U^{-}\left(\theta^{\prime}, \theta\right)$, on obtient une deuxième condition du premier ordre portant sur la dérivée $\varphi^{\prime}(\theta)$. En associant l'équation à laquelle satisfait $\varphi^{\prime}(\theta)$ à la condition initiale $\varphi(\theta)=0$, on aboutit alors à un problème de contrôle optimal consistant à maximiser l'espérance du bien être social sous les contraintes d'incitation et de participation. Dans ce problème de contrôle optimal, les variables $e(\theta)$ et $Q(\theta)$ sont les variables de commande et $\varphi(\theta)$ est la variable d'état dont l'évolution est donnée par la condition du premier ordre que satisfait $\varphi^{\prime}(\theta)$

Jean Jacques Laffont et Jean Tirole (1986) parviennent ainsi à la construction d’un mécanisme direct, révélateur et socialement optimal satisfaisant plusieurs propriétés dont les principales sont rappelées ici:

$1 /$ L'entreprise régulée de type $\theta$ choisit au sein du menu qui lui est proposé l'option correspondant à son vrai type $\theta$

2/ Les entreprises de type $\theta<\theta^{+}$fournissent un effort inférieur à celui de l'optimum de premier rang ;

3 / les entreprises de type $\theta>\theta_{-}$obtiennent une rente informationnelle positive : $U(\theta, \theta)>0, \forall \theta>\theta_{-}$;

4/ L'entreprise la plus efficace $\left(\theta=\theta^{+}\right)$choisit le contrat qui requiert un niveau d'effort maximal (i.e. optimum de premier rang); 5 / L'entreprise la moins efficace $(\theta=\theta)$ choisit le contrat qui exige le niveau d'effort le plus faible ;

$6 /$ Les entreprises dont l'inefficacité est intermédiaire $(\theta \in] \theta_{-}, \theta^{+}[)$choisissent un contrat à la puissance incitative intermédiaire.

\footnotetext{
${ }^{17}$ Le lecteur intéressé par un tour d'horizon complet des théories de la régulation est renvoyé au chapitre 27 de Mark Armstrong et David Sappington "Recent developments in the theory of regulation" in Handbook of Industrial Organization, vol. 3, 2007, North Holland, eds. M. Armstrong et M. Porter, 1557-1700.
} 


\section{IV.5 Prolongements du modèle de base}

Le modèle de base de Jean Jacques Laffont et Jean Tirole (1986) que nous venons d'esquisser et le monumental ouvrage qui a suivi (1993) ont eu un retentissement considérable, non pas tant dans l'application directe à la régulation des activités en monopole naturel, mais surtout en tant que guides intellectuels pour penser différents aspects de la régulation et ouvrir de nouvelles perspectives. Nous répertorions ici quelques aspects.

\section{a. Choix entre deux options}

En premier lieu, le régulateur peut chercher à réduire le menu d'options possibles. Une simplification possible consiste à réduire à deux le nombre d'options proposées à l'entreprise régulée. Au lieu de proposer un menu continu de contrats, comme au paragraphe précédent, le régulateur pourrait ne proposer à l'entreprise régulée qu'un choix entre deux contrats de puissances extrêmes: I'un, étant un contrat "cost plus", de puissance nulle où l'Etat rembourserait l'intégralité du dépassement de coût, l'autre étant un contrat "prix fixe", de puissance maximale où l'Etat ne rembourserait aucun dépassement de coût. Jean Jacques Laffont et Jean Tirole (2000) ont montré que si le choix était proposé entre deux mécanismes, de puissance respectivement faible et élevée, l'entreprise la plus efficace choisirait le mécanisme ayant la plus forte puissance, ce qui rejoint un résultat qualitatif obtenu dans le modèle de base. Une étude empirique de Philippe Gagnepain et Marc Ivaldi, 2002, consacrée aux effets de la régulation du transport urbain en France, parvient à la conclusion qu'un choix binaire entre deux contrats de puissances extrêmes conduirait à une amélioration substantielle du surplus social par rapport à ce que permet d'atteindre la régulation en place.

\section{b. Dynamique de la régulation et renégociation des contrats}

Dans un cadre dynamique où la relation entre le régulateur et l'entreprise régulée s'inscrit dans le temps, deux questions se posent avec acuité. D'une part, l'engagement temporel du régulateur est-il crédible ? D’autre part, comment éviter que l'entreprise régulée renonce à réaliser des investissements de long terme ? Raisonnons pour simplifier sur deux périodes.

La question de la crédibilité de l'engagement du régulateur se pose du fait de l'asymétrie informationnelle initiale. En effet, en présence d'une telle asymétrie, il n'est pas évident que le régulateur ne cherche pas à bénéficier en seconde période de l'information glanée à l'issue de la première période, concernant notamment le type de l'entreprise régulée, c'est-à-dire le paramètre d'antisélection. En effet, l'information au début de la seconde période devenant disponible pour le régulateur, celui-ci est en mesure de réaliser un optimum de premier rang, ce qui exigerait alors un effort plus élevé de l'entreprise régulée. Anticipant cela, l'entreprise régulée a intérêt à ne pas révéler au cours de la première période sa véritable productivité parce qu'une telle révélation conduirait le régulateur à exiger d'elle un plus grand effort. C'est une conséquence de "l'effet de cliquet" que nous examinons plus loin. De ce fait, un engagement initial du régulateur à appliquer le même contrat sur les deux périodes paraît peu crédible. Se pose alors la question de la nature d'un possible réaménagement contractuel sur les deux périodes. Cette question a été examinée par Jean Jacques Laffont et Jean Tirole (1990) et son traitement a consisté à examiner une forme contractuelle spécifique : le régulateur s'engage sur un même contrat au cours des deux périodes sauf si l'entreprise régulée demande au début de la seconde période une renégociation du contrat de première période. L'intérêt du régulateur serait donc de proposer en première période un contrat non susceptible de conduire à une renégociation ("renegotiationproof"). L'examen d'un tel contrat a fait l'objet d'un travail de Oliver Hart et Jean Tirole (1988) repris dans l'ouvrage de 1993 (chapitre 10). Un des résultats les plus marquants est qu'un contrat non renégociable entre un principal et un agent, ayant tous deux un facteur d'actualisation élevé, ne doit pas comporter des options "fortement séparatrices selon le type d'agent". Autrement dit, c'est en renonçant à l'équilibre séparateur du modèle à une période, c'est-à-dire un équilibre où chaque type d'agent se voit proposer un contrat spécifique, que le régulateur parvient à un contrat non renégociable dans un modèle à deux périodes.

Par ailleurs, une autre difficulté qu'introduit le cadre dynamique résulte à nouveau de l'effet cliquet ("ratchet effect") qu'on peut résumer comme suit. Si un investissement irréversible de l'entreprise régulée durant la première période induit une baisse de son coût, le régulateur peut être amené à réduire le transfert de seconde période. L'anticipation d'une telle possibilité par l'entreprise régulée peut suffire à la dissuader de réaliser l'investissement en question. Cette situation peut être d'autant plus préjudiciable que l'investissement est bénéfique du point de vue de la productivité de l'entreprise régulée. Jean Tirole (1986) a ainsi examiné la possibilité d'un sous investissement du prestataire dans le cadre d'une commande publique en s'appuyant sur un travail antérieur de Xavier Freixas, Roger Guesnerie et Jean Tirole (1985).

\section{c. Les enchères}

Une perspective pour accroître la puissance du mécanisme peut être obtenue en réduisant l'incertitude du régulateur sur l'efficacité de l'entreprise régulée (antisélection). Pour cela, Jean Jacques Laffont et Jean Tirole (1993) proposent d'introduire une enchère multidimensionnelle pour gagner le droit d'être le seul prestataire sur le marché régulé, comme cela se produit souvent dans le cas d'une adjudication publique. Mais, il ne s'agit pas simplement de sélectionner l'entreprise qui choisit le prix le plus faible. L'enchère multidimensionnelle dont il est question ici est plus subtile : elle porte à la fois sur le prix et sur le mécanisme, en l'occurrence un menu de partages des coûts. Le résultat de l'enchère révèlerait l'entreprise la plus efficace par le choix qu'elle fait de l'option au sein du menu proposé. Le chapitre 7 de l'ouvrage de 1993 est consacré à l'analyse de cette question et le chapitre 8 en analyse les aspects dynamiques. Là encore, le traitement de cette question s'avère d'une grande utilité dans toutes les situations où un donneur d'ordre public décide de choisir le prestataire pour la réalisation d'un service non pas sur ses seules caractéristiques intrinsèques, caractéristiques qu'au demeurant le donneur d'ordre ne connait pas totalement, mais plutôt en faisant révéler aux candidats à l'enchère leurs caractéristiques respectives par le choix qu'ils opèrent du coefficient de partage des coûts proposé par l'enchère.

\section{d. L'ouverture à la concurrence}

L'ouverture à la concurrence de certaines composantes des activités de réseaux a été une initiative importante dans l'évolution des processus de régulation depuis plus de trente ans à travers le monde. Par exemple, dans le domaine de l'électricité, des activités telles que la production et le commerce sont maintenant ouvertes à la concurrence tandis que les activités proprement dites de réseau (transport et distribution) restent des monopoles naturels. A partir d'une situation initiale d'un monopole public verticalement intégré et présent sur différentes activités, trois types de politiques publiques sont concevables: 1/ Réduire les barrières à l'entrée dans le secteur potentiellement concurrentiel et introduire une régulation permettant aux entrants d'accéder aux goulots d'étranglement ; 2 / Restructurer l'activité du monopole en le démantelant pour parvenir à des activités indépendantes soumises chacune à la concurrence ; $3 /$ Coordonner les politiques de la concurrence et de la régulation pour contrôler les activités du monopole. Le choix opéré entre ces modalités varie considérablement selon les activités et selon les pays. D’une part, la concurrence est davantage concevable dans certaines activités que dans d'autres. D'autre part, les vertus que les pays accordent à la concurrence varient beaucoup entre pays. Aux Etats-Unis, l'option 2 a 
parfois été choisie (démembrement d’ATT, voir David Encaoua et Philippe Koebel, 1987). En Europe, ce sont plutôt les options 1 et 3 qui ont été utilisées.

Prenons l'exemple de l'énergie électrique. Jusqu'à une période récente, le secteur de l'énergie électrique dans chaque pays membre de l'Union Européenne était caractérisé par la présence d'un opérateur historique, verticalement intégré, et en situation de monopole sur chaque marché national. Mais, comme le rappellent Claude Crampes et Thomas Olivier Léautier (2015), la situation a changé depuis la directive $n^{\circ}$ 96/92/CE du 19 décembre 1996 concernant les règles communes pour le marché intérieur de l'électricité. Le premier changement a entrainé la séparation des activités de réseau (transport et distribution) des activités potentiellement concurrentielles (production et commerce). Une nouvelle régulation a commencé à se mettre en place. Concernant les activités ouvertes à la concurrence, la première question a été celle de l'accès au réseau de transport. Deux solutions étaient possibles. La première consistait à définir des droits physiques d'accès et à les échanger entre pays membres. La deuxième solution, proposée par William Hogan (1992), consiste à créer des droits financiers de transport, qui payent à leur détenteur la différence de prix aux extrémités d'une ligne. De ce fait, un producteur détenteur d'un instrument financier qui compense la différence de prix entre deux pays membres, n'a plus besoin de se procurer une capacité physique sur chaque ligne. Laquelle de ces deux solutions est préférable ? Une contribution de Paul Joskow et Jean Tirole (2000) répond à cette question en montrant que "les droits financiers sont en général préférables aux droits physiques : un producteur détenteur de droits physiques pourrait décider de ne pas utiliser l'ensemble de la capacité acquise pour augmenter ses profits, ce qui n'est pas possible s'il détient des droits financiers" (Claude Crampes et Thomas Olivier Léautier, 2015). Une autre question a émergé à la suite de l'apparition de nouvelles unités de production intervenant sur des connexions ou des lignes marchandes en vendant leur production sur le marché de gros. Comment déterminer le prix des interconnexions sur ces lignes marchandes? A nouveau, un travail de Paul Joskow et Jean Tirole (2005) apporte un élément de réponse. L'optimum social consiste à choisir la capacité d'une telle ligne de sorte que la différence de prix entre les deux extrémités de la ligne soit égale au coût marginal de la capacité. Mais la concurrence imparfaite sur la liaison marchande empêche en fait la réalisation de cet optimum, du fait du choix stratégique de la capacité par le premier entrant sur la liaison, les promoteurs de telles lignes étant naturellement incités à exercer leur pouvoir de marché. Dès lors, les autorités de la concurrence, comme celles de la régulation doivent veiller à ce que des barrières à l'entrée ou des pratiques abusives ne s'exercent pas sur les lignes marchandes. En Europe, ces lignes sont à présent traitées comme des facilités essentielles et sont de ce fait soumises au droit de la concurrence.

De multiples autres questions très complexes se posent évidemment à propos d'autres activités. Prenons l'exemple d'une activité en pointe, celle des moteurs de recherche sur Internet. L'opérateur dominant Google est soupçonné d'induire par l'élargissement considérable de son périmètre d'activités l'impossibilité de maintenir un niveau de concurrence satisfaisant. Les solutions publiques concevables face à une telle accusation sont à nouveau de l'un des trois types mentionnés plus haut. Les solutions proposées en Europe et aux Etats-Unis sont contrastées. En novembre 2014, le Parlement Européen exprimait un vote d'appréciation en faveur de l'option 2, à savoir un démantèlement de Google en séparant l'activité de moteur de recherche de ses autres activités commerciales. Quelque temps auparavant, en Janvier 2013 aux Etats-Unis, la Federal Trade Commission dédouanait pourtant Google de l'accusation de créer des barrières à l'entrée dans les sites de recherche en favorisant son propre site au détriment de ceux des concurrents, car selon la FTC, "Google's search algorithms - even those that may have had the effect of harming individual competitors - could be plausibly justified as innovations that improved Google's product" (Mariniello, 2014). Comment expliquer ces différences d'appréciation ? Sont elles dues au très faible pourcentage d'entreprises européennes parmi les leaders mondiaux de l'industrie du numérique, que l'Europe cherche à corriger en imposant une solution que les Etats Unis réprouvent? Ou bien, traduisent-elles plus simplement l'existence de comportements réellement abusifs sur certains continents et pas dans d'autres, seuls les premiers étant à condamner ? C'est évidemment une question d'actualité difficile, au croisement du droit de la concurrence et du droit de régulation sectorielle, l'articulation entre ces deux droits ne se faisant pas sans difficultés (Philippe Choné, 2006).

\section{6 Institutions : capture et incitations}

\section{a. Théorie de la capture}

L'idée que la régulation publique d'une entreprise puisse servir les intérêts de l'entreprise régulée plutôt que ceux de la collectivité remonte à George Stigler (1971). La littérature sur la théorie de la capture n'a cessé de prospérer depuis, mais Jean Jacques Laffont et Jean Tirole (1991) ont cherché à mieux comprendre la notion de capture en s'inspirant d'un travail antérieur relevant de la théorie des organisations (Jean Tirole, 1986). Un élargissement de la théorie traditionnelle de l'agence a été réalisé en introduisant trois niveaux hiérarchiques, un principal, un superviseur et un agent. Adaptés au cadre de la régulation économique, les trois niveaux sont respectivement l'Etat qui définit les règles en explicitant un mécanisme (rôle du Principal), l'Agence de Régulation à qui l'Etat délègue le soin d'appliquer le mécanisme (rôle de Superviseur) et l'Entreprise Régulée (l'Agent). Le cadre d'analyse est toujours celui d'un mécanisme incitatif en information asymétrique : le principal et le superviseur ne connaissent ni le type de l'entreprise ni n'observent son effort pour réduire le coût de production. Supposons pour simplifier que l'agent ne soit que de deux types possibles : coût élevé (agent inefficace) ou coût faible (agent efficace). Face à un mécanisme du type de celui exposé plus haut, un agent inefficace aurait intérêt à se révéler en tant que tel au superviseur, mais ce n'est pas le cas pour un agent efficace. Celui-ci pourrait avoir intérêt à "soudoyer" le superviseur pour qu'il ne révèle pas au principal son type. C'est notamment le cas si la rente informationnelle que s'approprie l'agent efficace est suffisamment importante pour qu'une part de celle-ci, versée comme "pot de vin" au superviseur, suffise à inciter celui-ci à ne pas reporter au principal le véritable état de l'entreprise régulée. Autrement dit, une collusion entre l'agent et le superviseur est possible, sans que le principal n'en soit informé, ce qui explique in fine une possibilité de capture du principal par l'agent, via le superviseur. Un moyen d'éviter une telle collusion entre le superviseur et l'agent, serait de mettre en place un mécanisme moins puissant qui réduise, autant que faire se peut, la rente informationnelle que le mécanisme octroie à la firme la plus efficace. Afin d'éviter cette éventuelle capture, une solution pour le principal pourrait donc consister à affaiblir la puissance du mécanisme, par exemple en accroissant la part du financement par la puissance publique. Cela implique, entre autre, que la mise en place d'un mécanisme plus faible ne doit pas nécessairement être considérée comme un défaut de conception dans la mesure où un tel mécanisme peut être une réponse appropriée au risque de collusion. Mais il faut garder à l'esprit qu'un mécanisme plus faible induit inexorablement un moindre effort pour réduire le coût.

\section{b. La régulation à la lumière des institutions}

Les problèmes institutionnels que rencontre la régulation économique peuvent être appréhendés de manière bien plus générale que ne le fait la théorie de la capture. Plutôt que de procéder à une simple généralisation de la théorie de l'agence comme cela a été rappelé au paragraphe précédent, Jean Tirole et Eric Maskin (2004) adoptent une approche d'économie politique visant un objectif beaucoup plus 
large. Les auteurs se sont intéressés aux propriétés de différentes configurations possibles de l'organisation de la vie en collectivité selon les pouvoirs et les objectifs de trois types d'acteurs: i/ les hommes politiques exerçant leurs prérogatives selon les principes d'une démocratie par délégation: ils sont élus par les citoyens et responsables de leurs actes auprès des électeurs lors d'une éventuelle réélection; ii/ les citoyens exerçant leurs prérogatives selon les principes d'une démocratie directe, comme c'est par exemple le cas lors de consultations périodiques par referendums; iii/ les juges exerçant leurs prérogatives selon les principes du droit : ils sont non élus et non responsables de leurs décisions auprès des citoyens car appliquant strictement le droit. Trois modalités d'organisation de la vie collective sont ainsi examinées: i/ la démocratie représentative par délégation du pouvoir à des représentants politiques, telle que nous la connaissons; ii/ la démocratie directe par recours au référendum, et iii/ la démocratie des juges fondée sur le droit des contrats et le pouvoir de les faire respecter. Pour pouvoir comparer ces modalités, différentes hypothèses sur les objectifs des agents sont nécessaires. Par exemple, un homme politique à qui les citoyens ont délégué un pouvoir, est supposé mû par l'un de deux objectifs conflictuels possibles. Le premier est de promouvoir ce qui est dans l'intérêt des citoyens, du moins ce qui en est perceptible au travers des sondages d'opinion. Le deuxième objectif, qu'on ne peut à priori écarter, est de bénéficier personnellement des privilèges qu'octroie le statut de représentant de la puissance publique. L'occurrence d'une réélection permet alors aux citoyens d'exercer deux effets positifs. Premièrement, ils décident de réélire ou non l'homme politique, sachant qu'ils n'observent pas parfaitement les efforts de ce dernier dans l'accomplissement du premier objectif. C'est la dimension d'aléa moral implicite à la fonction de contrôle politique qu'exerce une réélection. Deuxièmement, une réélection donne aux citoyens le pouvoir de distinguer les hommes politiques déviants, c'est-à-dire ceux qui leur paraissent privilégier le second objectif. C'est alors une dimension de sélection adverse implicite à la fonction de contrôle politique qu'exerce une réélection. On retrouve là une problématique issue de la dynamique des contrats, où l'occurrence d'une réélection révèle des caractéristiques d'agents, non connues auparavant. Mais les réélections ont également deux effets négatifs. Premièrement, elles peuvent inciter l'homme politique désireux de se faire réélire, à "flatter" son électorat au détriment de l'intérêt de la société dans son ensemble. Deuxièmement, le principe même d'une majorité de votants pour décider du résultat de la réélection induit l'homme politique à sous évaluer l'intérêt des minoritaires, ce qui nuit à la nécessaire cohésion sociale. Une constitution spécifie ainsi "qui fait quoi" et la comparaison des trois modalités d'organisation de la vie collective selon le critère du surplus social global peut être entreprise. Nous n'entrerons pas davantage ici dans les subtilités de ce modèle d'économie politique sinon pour souligner une intéressante parenté avec la question du choix des institutions les plus aptes à assurer au mieux la régulation des monopoles naturels. Plutôt que confier le soin de réglementer les entreprises publiques à des représentants du monde politique, comme cela a longtemps été pratiqué, notamment en France, une solution préférable et largement utilisée consiste à mettre en place des agences de réglementation indépendantes, dirigées par des experts eux-mêmes indépendants aussi bien des entreprises régulées que de la puissance publique, et dont les décisions peuvent être examinées en appel auprès de juges. Une autre parenté peut être soulignée. Comme dans le modèle d'économie politique qui vient d'être évoqué, la dynamique temporelle que constitue le principe d'une réélection, trouve son équivalent en théorie de la régulation par le biais de la renégociation des contrats, examinée plus haut. Une réélection est ainsi assimilée à une possibilité de renégociation. Enfin, une question plus générale encore se réfère au recours à des incitations pécuniaires comme instrument de la régulation économique. Dans quelle mesure des incitations pécuniaires, en tant qu'instruments externes de motivation, ne vont-elles pas à l'encontre des motivations intrinsèques des dirigeants des entreprises régulées ? Pour ne citer qu'une illustration dans un tout autre champ, une rémunération plus élevée incite-t-elle un chercheur à fournir un plus grand effort de recherche ou au contraire n'est-elle pas plutôt contreproductive, au sens où c'est la passion pour la recherche qui constitue l'incitation de base et non le taux de rémunération que le chercheur reçoit pour exercer ce métier? Cette question des vertus relatives des motivations extrinsèques et intrinsèques est au cœur de nombreux débats entre économistes d'une part et sociologues et psychologues d'autre part. Les premiers ont longtemps pensé que dans le cadre d'une relation d'agence traditionnelle, l'offre par le principal d'une compensation monétaire par le biais d'un transfert financier incite l'agent à réaliser l'effort approprié pour obtenir le résultat désiré par le principal. Il s'agit alors d'une motivation extrinsèque. Les seconds mettent plutôt l'accent sur des motivations intrinsèques : l'esprit public, la transparence et la rigueur, voire même le plaisir que peut éprouver un agent public dans l'accomplissement de son activité, pour peu que l'environnement y soit favorable, seraient des moteurs de son engagement. Ce type de débat a évidemment de multiples ramifications dans le champ social. Par exemple, doit-on maintenir un système généreux de protection des chômeurs dans un objectif de justice sociale en espérant que leur préférence pour une activité salariée est suffisamment forte pour qu'ils persistent dans la recherche active d'un emploi, ou doit-on au contraire réduire le niveau de la protection sociale pour que la recherche d'un emploi devienne plus impérieuse ? Ces questions sont dans le droit fil d'un travail de Roland Benabou et Jean Tirole (2003), que les lecteurs de la Review of Economic Studies ont jugé comme étant l'article le plus novateur de l'année. Les auteurs ont cherché à comprendre dans quelles circonstances les motivations extrinsèques et intrinsèques pouvaient entrer en conflit ou au contraire agir dans le même sens. A nouveau, la parenté avec la régulation économique est intéressante à saisir. Un service public régulé doit-il bénéficier de transferts financiers pour l'inciter à être efficace ou l'efficacité n'est elle que la résultante de l'esprit public sensé animer ses dirigeants ? Vaste question à laquelle il est bien entendu difficile pour ne pas dire impossible d'apporter une réponse catégorique, mais à laquelle l'article cité apporte une intéressante contribution, débordant largement l'étroite perspective de l'homo economicus !

\section{c. Croyances, efforts et modèles d'organisation collective}

Les performances collectives sont elles le reflet des croyances individuelles en l'effet bénéfique de l'effort, comme des représentations courantes de l'idéologie américaine le laissent penser ? En d'autres termes, la croyance collective en les vertus de l'effort individuel dériveelle de l'observation des performances ou n'est-ce qu'un mythe forgé par l'idéologie ? Alors que l'examen de cette question de fond est généralement le fruit d'analyses sociologiques, psychologiques, historiques, politiques, voire même d'analyses croisées entre ces différentes disciplines, un très intéressant article de Roland Benabou et Jean Tirole (2006), développe une perspective d'économie politique originale tout en multipliant précisément les références à ces différentes approches des sciences sociales. Partant du contraste entre, d'une part, une vision du modèle américain où la responsabilité individuelle et l'effort priment sur l'attente d'une justice publique réparatrice par les effets de la redistribution et, d'autre part, celle d'un modèle européen où la croyance que les déterminismes sociaux l'emportent sur les illusions d'un monde où une pure méritocratie serait la norme, Roland Benabou et Jean Tirole parviennent à montrer que les deux situations correspondent en fait à deux types d'équilibre possibles, c'est-à-dire des situations où les croyances et les modalités d'intervention publique se renforcent mutuellement. Pour illustrer, partons de l'exemple américain. Si une majorité de citoyens pense que le succès individuel dépend de son propre effort, de tels citoyens chercheront à minimiser le rôle de la redistribution en refusant un poids excessif de la fiscalité. De plus, des citoyens anticipant un faible effet de la redistribution du fait de l'insuffisance des moyens publics, tenteront de donner plus de poids à leurs propres motivations individuelles. Les croyances individuelles (qu'elles soient idéologiques ou religieuses) en les vertus de l'effort de chacun et le système politique favorisant les incitations individuelles et en évitant de trop intervenir dans le fonctionnement des marchés (ce qui n'équivaut pas au laissez faire) deviennent alors des facteurs complémentaires qui se renforcent et se stabilisent, donnant naissance à un équilibre de type américain. Mais, l'équilibre de type européen est tout autant 
soutenable. Un plus grand pessimisme quand à la réalité de la méritocratie comme principe régulateur conduit à un interventionnisme plus important des pouvoirs publics pour corriger les inégalités et inversement une forte autorité centrale avec les moyens qu'elle suppose réduit les efforts individuels ou plus précisément la croyance en les vertus de ces efforts. Voilà encore un type d'analyse, mettant en relation les croyances et le système de gouvernance, qui nous éloigne des modèles traditionnels de l'homo economicus.

\section{Conclusion}

Au terme de cet essai de mise en perspective de quelques uns des travaux qui ont valu à Jean Tirole le prix Nobel 2014, deux appréciations générales peuvent être avancées.

Premièrement, il s'agit indéniablement de contributions d'une très grande portée sur les plans de la réflexion et de l'analyse, qui ont conduit à un profond renouveau de pans entiers de l'économie. Nous avons privilégié dans cet article les contributions touchant à l'économie industrielle, à l'économie de l'innovation et à l'économie de la régulation, mais ce faisant, nous avons omis de très importantes contributions dans de multiples autres domaines, tels que l'économie de la banque et de la finance, la théorie des organisations, l'économie de l'environnement, l'économie et la psychologie des comportements, la macroéconomie et l'économie du travail, la finance d'entreprise, la régulation bancaire, etc. Manifestement Jean Tirole est un économiste prolixe et de grand talent. Non seulement il parvient à faire reculer les frontières de la connaissance, mais également et surtout, il n'hésite pas à remettre en question les principes traditionnels sous jacents aux approches de l'homo economicus, pour parvenir à cet élargissement du savoir. Son succès n'est pas seulement dû à une maitrise exceptionnelle des outils de modélisation, tels que la théorie des jeux et la théorie des incitations. Il s'explique surtout par le fait qu'il explore les questions en véritable économiste, c'est-à-dire en représentant d'une discipline qui cherche d'abord à identifier pour chaque question les points les plus saillants, en s'aidant de considérations historiques et factuelles, de manière à dégager une véritable problématique économique qui, associée à une forte intuition de base, permet in fine de recevoir un traitement analytique approprié. C'est une démarche que l'on retrouve quasiment dans toutes ses contributions et qui explique que celles-ci ont eu finalement un rôle précurseur dans nombre de travaux contemporains. Elles témoignent toutes d'une formidable ouverture aux progrès de la connaissance des phénomènes sociaux, de quelque discipline que proviennent ces progrès. Ce que témoigne encore la création récente de I'IAST (Institute for Advanced Studies of Toulouse), Laboratoire d'Excellence qu'il a fondé en 2011 à Toulouse, qui se réclame ouvertement du dialogue interdisciplinaire, seul à même de rompre les barrières artificielles entre les différentes approches de l'homme et de la société. ${ }^{18}$ Pour exprimer la deuxième appréciation, il nous faut partir d'un argument développé par le politologue américain Donald Stokes (1997) qui a cherché à établir des liens entre les travaux de recherche selon qu'ils ressortissent de la science ou de l'innovation. Pour cela, il propose de classer les travaux de recherche selon deux critères. Le premier consiste à savoir si une recherche poursuit ou non un objectif de connaissance fondamentale, c'est-à-dire de faire reculer la frontière des connaissances de base. Le deuxième consiste à savoir si une recherche poursuit ou non l'objectif de résoudre un problème concret spécifique. Donald Stokes parvient de la sorte à une matrice $2 \times 2$ dont il nomme chacune des cases du nom d'un savant emblématique. La première case (oui, non) regroupe les travaux de recherche qui poursuivent un objectif de connaissance fondamentale sans aucune considération relative à la résolution d'un problème concret spécifique. Donald Stokes la nomme quadrant de Niels Boehr, du nom du physicien Danois, connu pour sa contribution à l'édification de la mécanique quantique. La deuxième case (non, oui) regroupe les travaux de recherche qui ne poursuivent aucun objectif de connaissance fondamentale mais cherchent simplement à résoudre un problème spécifique. Donald Stokes la nomme quadrant de Thomas Edison, du nom de l'inventeur du filament de l'ampoule électrique. La troisième case (oui, oui) regroupe les travaux de recherche qui poursuivent à la fois un objectif de connaissance de base et celui de résoudre un problème spécifique. Donald Stokes nomme cette case "quadrant de Louis Pasteur", du nom du chimiste français du $19^{\text {ème }}$ siècle qui fut un pionnier de la microbiologie pour mettre au point un vaccin contre la rage. $^{19}$ La deuxième appréciation à l'égard des travaux de Jean Tirole peut à présent être énoncée. Ils correspondent selon nous au quadrant de Louis Pasteur, dans la mesure où, comme on espère en avoir convaincu le lecteur, ils satisfont à la fois l'objectif de faire avancer la théorie économique en faisant reculer la frontière de nos connaissances, et celui de suggérer des réponses appropriées à des problèmes économiques contemporains, souvent d'ailleurs en partant directement de l'un de ces problèmes pour en faire un objet d'analyse.

\section{Bibliographie}

Aghion, P., C. Harris, P. Howitt, \& J. Vickers (2001), Competition, Imitation and Growth with Step-by-Step Innovation, Review of Economic Studies, 68, 467-492

Aghion, P. \& R. Griffith, 2005, Competition and Growth, Reconciling Theory and Evidence, MIT Press

Aghion, P., U. Akcigit \& P. Howitt (2014), What Do We Learn From Schumpeterian Growth Theory?, in Handbook of Economic Growth, Vol.

2, Eds. P. Aghion and S. Durlauf, 515-563

Armstrong, M., S. Cowan, \& J. Vickers (1994), Regulatory Reform, Economic Analysis and British Experience, MIT Press

Armstrong, M. \& David Sappington (2007), Recent developments in the theory of regulation, in Handbook of Industrial Organization, vol. 3, 2007, North Holland, eds. M. Armstrong et M. Porter, 1557-1700

Averch, H. \& L. Johnson (1962), Behavior of the Firm under Regulatory Constraint, The American Economic Review, Vol. 52, 5, 1052-1069

Bain, J. (1956), Barriers to New Competition, Harvard University Press

Baron, D. \& R. Myerson (1982), Regulating a Monopolist with Unknown Costs, Econometrica, 50, 911-930

Baumol, W., J. Panzar \& R. Willig (1982), Contestable Markets and the Theory of industry Structure, Harcourt Brace Jovanovich

Benabou, R. \& J. Tirole (2002), Self-Confidence and Personal Motivation, Quarterly Journal of Economics, 117, 3, 871-915.

Benabou, R. \& J. Tirole (2003), Intrinsic and Extrinsic Motivation, The Review of Economic Studies, Vol. 70, 3, 489-520

Benabou, R. \& J. Tirole (2006), Belief in a Just World and Redistributive Politics, The Quarterly Journal of Economics, Vol. 121, 2, 699-746

Benabou, R. \& J. Tirole (2009), Over My Dead Body: Bargaining and the Price of Dignity, American Economic Review, Papers and

Proceedings, 99, 2, 459-465

Benkler, Y. (2006), Technology Policy, Cooperation and Human Systems Design, in The New Economics of Technology Policy, Ed. D. Foray, 337- 364

Boiteux, M. (1956), Sur la Gestion des Monopoles Publics Astreints à l'Equilibre Budgétaire, Econometrica, 24, 22-40

Bork, R. (1978), The Antitrust Paradox: A Policy at War with Itself, Basic Books, New York

\footnotetext{
${ }^{18}$ Cinq programmes interdisciplinaires sont en cours associant l'Economie, l'Histoire, la Biologie, le Droit, la Science Politique et la

Psychologie. Ce qui promet une belle moisson de travaux à venir et un véritable élargissement de nos œillères disciplinaires !

${ }^{19}$ Le dernier quadrant est sensé être vide car il ne correspond pas à un travail de recherche.
} 
Caillaud, B., R. Guesnerie, P. Rey, \& J. Tirole [1988], Government Intervention in Production and Incentives Theory: A Review of Recent Contributions, Rand Journal of Economies, 19, 1-26

Chamberlin, E. (1933), The Theory of Monopolistic Competition, Harvard University Press

Chiao, B., J. Lerner \& J. Tirole (2007), The Rules of Standard Setting Organizations: An Empirical Analysis, Rand Journal of Economics, Vol. $38,4,905-930$

Choné, P. (2006), L'articulation des politiques de concurrence et de regulation sectorielle, in Politiques de la Concurrence, Rapport CAE, 209-232

Coase, R. (1945), Price and Output Policy of State Enterprise: A Comment, Economic Journal, 55, 112-113

Coase, R. (1946), The Marginal Cost Controversy, Economica, 13, 169-182

Coase, R. (1960), The Problem of Social Cost, Journal of Law and Economics, 1, 1-44

Coase, R. (1972), Durability and Monopoly, Journal of Law and Economics, 15, 143-149

Cournot, A. A. (1838), Recherches sur les Principes Mathématiques de la Théorie des Richesses, réédité en 1974, Calmann-Lévy

Crampes, C., D. Encaoua \& A. Hollander (2006), Competition and Intellectual Property Rights in the European Union, in New Developments

in UK and EU Competition Policy, Eds. Roger Clarke and Eleanor Morgan, Edward Elgar, 202-231

Crampes, C. \& T. O. Léautier (2015), Jean Tirole: Imperfect Competition and Regulation of the Electric Power Industry, TSE Debate

Dasgupta, P. \& J. Stiglitz (1980, a), Industrial Structure and the Nature of Innovative Activity, Economic Journal, Vol. 90, 266-293

Dasgupta, P. \& J. Stiglitz (1980, b), Uncertainty, Industrial Structure and the Speed of R \& D, Bell Journal of Economics, 1-28

Debreu, G. \& H. Scarf (1967), A Limit Theorem on the Core of an Economy, International Economic Review, Vol.4, 3, 235-246

Denicolo, V. \& P. Zanchettin (2012), Leadership Cycles in a Quality-ladder Model of Endogenous Growth, Economic Journal 122, 618-650

Dixit, A. (1979), A Model of Duopoly Suggesting a Theory of Entry Barriers, Bell Journal of Economics, vol. 10, 20-32

Dixit, A. (1980), The Role of Investment in Entry Deterrence, Economic Journal, vol. 90, 95-106

Dorazelski, U. \& A. Pakes (2007), A Framework for Applied Dynamic Analysis in IO, in Handbook of Industrial Organization, Volume 3

Eds. M. Armstrong and R. Porter, 1889-1966

Dorazelski, U. \& M. Satterthwaite (2010), Computable Markov-Perfect Industry Dynamics, RAND Journal of Economics, 41 (2), 215 - 243,

Dupuit, J. (1854), Dictionnaire de l'Economie Politique, Vol. II, Paris, Coquelin et Guillaumin

Edgeworth, F. (1897), The Pure Theory of Monopoly, in Papers Relating to Political Economy, vol.1, Ed. F. Edgeworth, MacMillan (1925) translation of "La Teoria Pura del Monopolio", Giornale Degli Economisti, 40, 13-31

Encaoua, D. \& A. Jacquemin (1980), Degree of monopoly, indices of concentration and threat of entry, International Economic Review, Vol. $21, n^{\circ} 1,87-105$

Encaoua, D. (1983), Dynamique des prix et structure industrielle : Une analyse théorique et économétrique", Document de travail du Département des Affaires économiques, No. 10, Éditions OCDE, doi : 10.1787/483558181564

Encaoua, D. \& Paul Geroski (1986), Price Dynamics and Competition in Five O.E.C.D. Countries, Revue Economique de l'O.C.D.E., n ${ }^{\circ} 6$

Encaoua, D., P. Geroski \& A. Jacquemin (1986), Strategic Competition and the Persistence of Dominant Firms: A Survey, in New Developments in the Analysis of Market Structure, Eds: Joseph Stiglitz \& Frank Mathewson, MIT Press, 55-89

Encaoua, D. \& P. Michel (1986), La Dynamique des Prix Industriels en France, Economica

Encaoua, D. \& M. Moreaux (1987), Concurrence et monopole naturel: Une approche par la théorie des jeux, Annales d'Économie et de Statistique, No. 8, 89-116

Encaoua, D. \& P. Koebel (1987), Réglementation et déréglementation des télécommunications: Leçons anglo-saxonnes et perspectives d'évolution en France, Revue Economique, Vol. 38, 2, 475-520

Encaoua, D. (1991), Regulatory Reform: a European Perspective, International Journal of Industrial Organization, vol.9, 1991, 1-7

Encaoua, D. \& A. Hollander (2002), Competition Policy and Innovation, Oxford Review of Economic Policy, Vol. 18, 1, 63-79

Encaoua, D. \& D. Ulph (2005), Catching-up or Leapfrogging: The Effects of competition on Innovation and Growth, https://halshs.archivesouvertes.fr/halshs-00177643

Encaoua, D. (2006), Comments, in The New Economics of Technology Policy, Ed. D. Foray, 358-364

Encaoua, D. \& R. Guesnerie (2006), Politiques de la Concurrence, Rapport CAE, La Documentation Française

Encaoua, D. \& T. Madiès (2014), Dysfunctions of the Patent System and their Effects on Competition, in Patent Markets in the Global Knowledge Economy, Eds T. Madiès, D. Guellec \& J.C. Prager, Cambridge University Press, 125-171

Encaoua, D. (2014), Book Review, Competition Policy and Price Fixing, Journal of Economics, Vol. 4, 3, 235-246

Ericson, R. \& A. Pakes (1995), Markov-Perfect Industry Dynamics: A Framework for Empirical Work, The Review of Economic Studies, Vol. 62, No. 1, 53-82

Farrell, J. \& G. Saloner (1985), Standardization, Compatibility, and Innovation, Rand Journal of Economics, Vol. 16, 1, 70-83

Farrell, J. \& G. Saloner (1986), Installed Base and Compatibility: Innovation, Product Preannouncements, and Predation, The American

Economic Review, Vol. 76, 5, 940-955

Fisher, F. (1989), Games Economists Play: A Noncooperative View, The RAND Journal of Economics, Vol. 20, 1, 113-124

Freixas, X., R. Guesnerie, \& J. Tirole (1985), Planning under Incomplete Information and the Ratchet Effect, Review of Economic Studies, 52, 173-191

Fudenberg, D. \& J. Tirole (1983), Capital as a Commitment: Strategic Investment to Deter Mobility, Journal of Economic Theory, 31, 227250.

Fudenberg, D., R. Gilbert, J. Stiglitz \& J. Tirole (1983), Preemption, Leapfrogging and Competition in Patent Races, European Economic Review, 22, 3-31

Fudenberg, D. \& J. Tirole (1983) Learning by Doing and Market Performance, Bell Journal of Economics, 14: $522-530$.

Fudenberg, D. \& J. Tirole (1984), The Fat-Cat Effect, the Puppy-Dog Ploy, and the Lean and Hungry Look, The American Economic Review, vol.74, $n^{\circ} 2$, Papers and Proceedings, 361-366

Fudenberg, D. \& J. Tirole (1987), A Theory of Dynamic Oligopoly, III: Cournot Competition, European Economic Review, 31: 947-968

Fudenberg, D. \& J. Tirole (1991), Perfect Bayesian Equilibrium and Sequential Equilibrium, Journal of Economic Theory, 53, 236-260

Fudenberg, D. \& J. Tirole (1991), Game Theory, The MIT Press

Gabszewicz, J. (1994), La Concurrence Imparfaite, Collection Repères, La Découverte

Gagnepain, P. \& M. Ivaldi (2002), Incentive Regulatory Policies: The case of Public Transit Systems in France, Rand Journal of Economics, 33, 605-629

Gagnepain, P. (2001), La Nouvelle Théorie de la Régulation des Monopoles Naturels, Revue Française d'Economie, Vol. 15, 4, 55-110 
Greenstein, S. (2010), The Emergence of the Internet: Collective Invention and Wild Ducks, Industrial and Corporate Change Vol. 19, 5, 1521-1562

Harsanyi, J. (1967-68), Games with Incomplete Information played Bayesian Players, Management Science, 14, 159-182, 320-334, 486-502 Hart, O. \& J. Tirole (1990), Vertical integration and market foreclosure, Brookings Papers on Economic Activity, Vol. 1990: Microeconomics, Brookings Institution, 205-286

Hart, O. \& J. Tirole (1988), Contract Renegotiation and Coasian Dynamics, Review of Economic Studies, 55: 509-540

Hall, R. \& C. Hitch (1939), Price Theory and Business Behavior, Oxford Economic Papers, 2, 12-45

Henderson, H. \& I. Cockburn (1996), Scale, Scope, and Spillovers: The Determinants of Research Productivity in Drug Discovery, The RAND

Journal of Economics, Vol. 27, 1, 32-59

Hogan, W. (1992), Contracts networks for electric power transmission, Journal of Regulatory Economics, 4, $211-242$

Holmström, B. (1999), Managerial Incentive Problems: A Dynamic Perspective, Review of Economic Studies, 66, 169-182

Joskow, P. \& J. Tirole (2000), Transmission rights and market power on electric power networks, RAND Journal of Economics, 31, 2, 450-487

Joskow, P. \& J. Tirole (2005), Merchant Transmission Investment, Journal of Industrial Economics, 53, 2, 233-264

Kaplow, L. (2013), Competition policy and price fixing, Princeton University Press

Katz, M. \& C. Shapiro (1985), Network Externalities, Competition, and Compatibility, The American Economic Review, Vol. 75, 3, 424-440

Katz, M. \& C. Shapiro (1986), Technology Adoption in the Presence of Network Externalities, Journal of Political Economy, Vol. 94, 4, 822-

841

Kreps, D., \& R. Wilson (1982), Sequential Equilibrium, Econometrica, 50, 863-894

Kreps, D. \& M. Spence (1985), Modelling the Role of History in Industrial Organization and Competition, in Issues in Contemporary Microeconomics and Welfare, Ed. Georges Feiwel, MacMillan, 340-378

Laffont, J. - J. \& J. Tirole (1986), Using Cost Observation to Regulate Firms, Journal of Political Economy, Vol. 94, N³, Part 1, 614-641

Laffont, J. - J. \& J. Tirole (1988), The Dynamics of Incentive Contracts, Econometrica, 56, 1153-1175

Laffont, J. - J. \& J. Tirole (1990), Adverse Selection and Renegotiation in Procurement, Review of Economic Studies, 75, 597-626

Laffont, J. - J. \& J. Tirole (1991), The Politics of Government Decision Making: A Theory of Regulatory Capture, Quarterly Journal of

Economics, 106, 1089-1127

Laffont, J. - J. \& J. Tirole (1993), A Theory of Incentives in Procurement and Regulation, MIT Press, Edition 1998

Laffont, J. - J., P. Rey \& J. Tirole (1998 a), Network Competition: I. Overview and Non Discriminatory Pricing, The RAND Journal of

Economics, Vol. 29, 1, 1-37

Laffont, J. - J., P. Rey \& J. Tirole (1998 b) Network Competition: II. Price Discrimination, The RAND Journal of Economics, Vol. 29, 1, 38-56

Laffont, J. - J. \& J. Tirole (2000), Global Price Caps and the Regulation of Access, Brazilian Review of Econometrics, 20, 115-146

Lee, T. \& W. Wilde (1980), Market Structure and Innovation: A Reformulation, Quarterly Journal of Economics, 194, 429-436

Lerner, J. \& J. Tirole (2002), Some Simple Economics of Open Source, Journal of Industrial Economics 50, 197-234

Lerner, J. \& J. Tirole (2004), Efficient Patent Pools, American Economic Review, 94, 691-711

Lerner, J. \& J. Tirole (2005), The Scope of Open Source Licensing, Journal of Law, Economics and Organization, 21, 20-56

Lerner, J., P. Pathak \& J. Tirole (2006), The Dynamics of Open Source Contributors, American Economic Review, Papers and Proceedings, 96: 114-118

Lerner, J., M. Strojwas \& J. Tirole (2007), The Design of Patent Pools: The Determinants of Licensing Rules, Rand Journal of Economics, Vol. $38,3,611-625$

Lerner, J. \& J. Tirole (2013), Standard-Essential Patents, IDEI Working Paper 803, 2013, forthcoming Journal of Political Economy

Loeb, M. \& W. Magat (1979), A decentralized method for utility regulation, Journal of Law and Economics, 22, 399-404

Loury, G. (1979), Market Structure and Innovation, Quarterly Journal of Economics, 93, 395-410

Mariniello, M. (2014), Unbundling Google Users from Europe, Bruegel, Section Comments, Mariniello@bruegel.org

Mason, E. (1939), Price and Production Policies of Large-Scale Enterprise, The American Economic Review, Supplement 29, 61-74

Maskin, E. \& J. Tirole (1988a), A Theory of Dynamic Oligopoly I: Overview and Quantity Competition with Large Fixed Costs, Econometrica, 56: 549-570

Maskin, E. \& J. Tirole (1988b), A Theory of Dynamic Oligopoly II: Price Competition, Kinked Demand Curves, and Edgeworth Cycles,

Econometrica, 56, 571-600

Maskin, E. \& J. Tirole (2001), Markov Perfect Equilibrium, I: Observable Actions, Journal of Economic Theory, 100: 191-219

Maskin, E. \& J. Tirole (2004), The Politician and the Judge: Accountability in Government, The American Economic Review, Vol. 94, 4, 1034-

1054

Noel, M. (2007), Edgeworth Price Cycles, Cost-Based Pricing, and Sticky Pricing in Retail Gasoline Markets, Review of Economics and Statistics, 89, 324-334

Pakes, A. \& P. McGuire (1994), Computing Markov Perfect Nash Equilibrium: Numerical Implications of a Dynamic Differentiated Product

Model, Rand Journal of Economics, 25, 555-589

Ramsey, F. (1927), A Contribution to the Theory of Taxation, Economic Journal, 37, 47-61

Reinganum, J. (1981), Dynamic Games of Innovation, Journal of Economic Theory, 25, 21-41

Reinganum, J. (1982), A dynamic game of R and D: Patent protection and competitive behavior. Econometrica , 50, 671-688.

Rey, P. \& J. Tirole (1986), The Logic of Vertical Restraints, The American Economic Review, Vol. 76, 5, 921-939.

Rey, P. \& J. Tirole (2007), A Primer on Foreclosure," in Handbook of Industrial Organization, vol.3, Eds. M. Armstrong et R.H. Porter, North Holland, p. 2145-2220

Rey, P. \& J. Tirole (2013), Cooperation vs. Collusion: How Essentiality Shapes Co-opetition, IDEI, Working Paper

Rochet, J. - C. \& J. Tirole (2003), Platform Competition in Two-Sided Markets, Journal of the European Economic Association 1, $990-1029$

Rochet, J. - C. \& J. Tirole (2006), Two-Sided Markets: A Progress Report, RAND Journal of Economics, 35, 645-667

Rochet, J. - C. \& J. Tirole (2008), Competition Policy in Two-Sided Markets, with a Special Emphasis on Payment Cards, in Handbook of Antitrust Economics, Ed. P. Buccirossi, MIT Press, 543-582

Sappington, D. (1982), Optimal Regulation of Research and Development under Imperfect Information, Bell Journal of Economics, 13, 354368

Sappington, D. \& D. Weisman (2010), Price Cap Regulation: What have we Learned from 25 years of Experience in the Telecommunications Industry? Journal of Regulatory Economics ,38, 227-257

Scherer, M. (1980), Industrial Market Structure and Economic Performance, $2^{\text {nd }}$ edition, Rand Mc Nally 
Selten, R. (1975), Re-examination of the Perfectness Concept for Equilibrium Points in Extensive Games, International Journal of Game Theory, vol. 4, 25-55

Shapiro, C. (1989), The Theory of Business Strategy, The RAND Journal of Economics, vol. 20, $\mathrm{n}^{\circ} 1,125-137$

Shapiro, C. (2001), Navigating the Patent Thicket: Cross Licenses, Patent Pools and Standard Setting, in Innovation Policy and the Economy, Eds. A. Jaffe and J. Lerner, MIT Press

Spector, D. (2006), Analyse économique et sécurité juridique : entre règles per se et règles de raison, in Politiques de la Concurrence,

Rapport CAE, 271-285

Spence, M. (1977), Entry, Capacity Investment and Oligopolistic Pricing, Bell Journal of Economics, vol. 8, 534-544

Spence, M. (1979), Investment Strategy and Growth in a New Market, Bell Journal of Economics, vol. 10, 1-19

Stigler, G. (1971), The Theory of Economic Regulation, Bell journal of Economics, 2, 3-21

Stokes, D. (1997), Pasteur's Quadrant, Basic science and Technological Innovation, Brookings Institution Press

Sutton, J. (1998), Technology and Market Structure, MIT Press

Sutton, J. (2007), Market Structure: Theory and Evidence, in Handbook of Industrial Organization, vol.3, Eds. M. Armstrong et R.H. Porter,

North Holland, 2303-2368

Sweezy, P. (1939), Demand under Conditions of Oligopoly, Journal of Political Economy, 47, 568-573

Tirole, J. (1983), Jeux Dynamiques: un Guide de I'Utilisateur, Revue d'Economie Politique, 93: 551-575

Tirole, J. (1985), Concurrence Imparfaite, Economica

Tirole, J. (1986), Hierarchies and Bureaucracies: on the Role of Collusion in Organizations, Journal of Law, Economics and Organization, 181-214

Tirole, J. (1986), Procurement and Renegotiation, Journal of Political Economy, 94, 235-259

Tirole, J. (1988), The Theory of Industrial Organization, The MIT Press

Tirole, J. (2005), The Analysis of Tying Cases: A Primer, Competition Policy International, vol.1, 1, 1-25

Tirole, J. (2012), La Régulation ou l'Economie Publique Moderne, La lettre de l'Autorité, N 54, février 2007 reproduit in 1997-2012,

Du monopole des Télécom à la Révolution Numérique, 15 ans de régulation, $\mathrm{n}^{\circ}$ spécial de l'ARCEP, Janvier

Vogelsang, I. \& J. Finsinger (1979), Regulatory Adjustment Process for Optimal Pricing by Multiproduct Monopoly Firms, Bell Journal of

Economics, 10, 157-171

Von Hippel, E. (1988), The Sources of Innovation, Oxford University Press

Von Hippel, E. (2006), Adapting Policy to User-Centered Innovation, in The New Economics of Technology Policy, Ed. D. Foray, $327-336$

Walras, L. (1883), Théorie Mathématique de la Recherche Sociale, réimprimé en 1964, Osnabrück, Otto Zeller

Whinston, M. (1990), Tying, Foreclosure and Exclusion, The American Economic Review, vol. 80, 4, 837-859

Zimmerman, P., J. Yun \& C. Taylor (2013), Edgeworth price cycles in gasoline: Evidence from the United States. Review of Industrial

Organization 42, 297-320. 